\title{
Caerulomycin and Collismycin Antibiotics Share a trans Flavin-Dependent Assembly Line for
}

\section{2,2'-Bipyridine Formation and Sulfur Fate Differentiation}

Bo Pang, ${ }^{1, \#}$ Rijing Liao, ${ }^{2, \#}$ Zhijun Tang, ${ }^{1 \#}$ Shengjie Guo, ${ }^{1}$ Zhuhua Wu, ${ }^{1}$ and Wen Liu ${ }^{1,3, *}$

${ }^{1}$ State Key Laboratory of Bioorganic and Natural Products Chemistry, Center for Excellence in

Molecular Synthesis, Shanghai Institute of Organic Chemistry, University of Chinese Academy of Sciences, 345 Lingling Road, Shanghai 200032, China;

${ }^{2}$ Shanghai Institute of Precision Medicine, Ninth People's Hospital, Shanghai Jiao Tong University School of Medicine, Shanghai 200125, China;

${ }^{3}$ Huzhou Center of Bio-Synthetic Innovation, 1366 Hongfeng Road, Huzhou 313000, China;

* To whom correspondence should be addressed: Shanghai Institute of Organic Chemistry, Chinese Academy of Sciences, 345 Lingling Rd., Shanghai 200032, China. Wen Liu, Email: wliu@sioc.ac.cn, Tel: 86-21-54925111, Fax: 86-21-64166128

\# These authors equally contribute to this work. 


\section{ABSTRACT:}

Linear nonribosomal peptide synthetases (NRPSs) and polyketide synthases (PKSs) template the modular biosynthesis of numerous nonribosomal peptides, polyketides and their hybrids though assembly line chemistry. This chemistry can be complex and highly varied, and thus challenges the understanding in the diverse polymerization processes of amino acid and carboxylate monomers programmed by various NRPSs and PKSs in nature. Here, we report that caerulomycin and collismycin peptide-polyketide hybrid antibiotics share an unusual assembly line that involves NRPS activity to recruit a flavoprotein acting in trans and catalyze $\mathrm{C}-\mathrm{C}$ bond formation and heterocyclization during 2,2'-bipyridine formation. Simultaneously, this assembly line provides dethiolated and thiolated 2,2'bipyridine intermediates through differential treatment of the sulfhydryl group arising from L-cysteine incorporation. Subsequent L-leucine extension, which does not contribute any atoms to either caerulomycins or collismycins, plays a key role in sulfur fate determination by selectively advancing one of the two 2,2'-bipyridine intermediates down a path to the final products with or without sulfur decoration. These findings further the appreciation of assembly line chemistry and will facilitate the development of related molecules using synthetic biology approaches. 


\section{INTRODUCTION}

Linear nonribosomal peptide synthetases (NRPSs) and polyketide synthases (PKSs) are often large molecular machines that are composed of multidomain modules ${ }^{1-6}$. They have evolved through functional unit (e.g., protein, module or domain) combination and variation to afford various assembly lines that program diverse polymerization and modification processes using amino acids, short carboxylic acids or both as monomers. Assembly line chemistry can be complex and highly varied, and thereby challenges our understanding of how nature creates numerous nonribosomal peptides, polyketides and hybrids thereof that historically play critical roles in medicinal chemistry and chemical biology. The biosynthesis of 2,2'-bipyridine, the core structure of a large class of synthetic bidentate metal-chelating ligands with a variety of applications in many areas of chemistry ${ }^{7,8}$, occurs via such a process.

2,2'-Bipyridine natural products include caerulomycins (e.g., CAE-A, Fig. 1), a group of structurally related antibiotics that possess potent immunosuppressive activity ${ }^{9-13}$. While the process through which 2,2'-bipyridine is formed remains poorly understood, we and others unraveled the biogenesis of CAEs and identified a NRPS-PKS assembly line that contains three modular proteins, i.e., CaeA1, CaeA2 and CaeA3, to template a peptide-polyketide hybrid skeleton for 2,2'-bipyridine formation (Fig. 1) ${ }^{14-16}$. CaeA1, a bifunctional enzyme composed of a peptidyl carrier protein (PCP) and an adenylation (A) domain, was supposed to prime picolinyl, which provides the unmodified pyridine unit (Ring B) of CAEs, as the starter unit for molecular assembly. CaeA2 is a hybrid protein, with a typical PKS module containing a ketosynthase (KS), acyltransferase (AT), and acyl carrier protein (ACP) and an 
atypical NRPS module containing a condensation/cyclization (Cy) domain, A domain, PCP and terminal $\mathrm{C}(\mathrm{Ct})$ domain. Logically, this protein can incorporate malonyl-S-CoA and L-cysteine in tandem and associate CaeA1 to provide the essential building atoms for constructing the di- or trisubstituted pyridine unit (Ring A) of CAEs ${ }^{14,17,18}$. As proposed, the PKS module of CaeA2 catalyzes two-carbon elongation and installs the atoms C3 and C4 following C2, the carboxyl carbon of the starter picolinyl unit. The atypical NRPS then catalyzes L-cysteine extension, which is unique and occurs at $\mathrm{C} \beta$ of the L-cysteine residue to form a C-C bond, instead of it's $\alpha$-amino group for amide bond formation as usual, and thus provides $\mathrm{C} 5, \mathrm{C} 6$ and $\mathrm{N} 1$ as well as exocyclic $\mathrm{C} 7$ after heterocyclization. To our knowledge, peptidyl elongation through a C-C linkage lacks precedent in NRPS catalysis. The domains of CaeA3 are organized as C-A-PCP for L-leucine extension ${ }^{14,15}$. Despite not contributing any atoms in the mature products, CaeA3 activity is necessary for the formation of 2,2'-bipyridinyl-L-leucine (1), an off-line intermediate that can be specialized to individual CAE members after L-leucinyl removal ${ }^{19-23}$. If $\mathbf{1}$ is the direct product of CaeA1, CaeA2 and CaeA3, 2,2'bipyridine formation could occur in this assembly line through an unprecedented dethiolation-coupled heterocyclization process that involves NRPS-mediated C-C bond formation.

Interestingly, homologs of the above modular synth(et)ases CaeA1, CaeA2 and CaeA3 (i.e., ColA1a and ColA1b, ColA2 and ColA3) were observed in the biosynthetic pathway of collismycins (e.g., COL-A, Fig. 1) ${ }^{14,24,25}$, the 2,2'-bipyridine antibiotics differing from CAEs with a sulfur-containing group at C5 of Ring A. Most likely, COLs share a similar assembly line for 2,2'-bipyridine formation in which, however, the dethiolation step can be skipped to retain the sulfur-containing group during assembly of the completely identical substrates picolinic acid, malonyl-S-CoA, L-cysteine and L- 
leucine ${ }^{16}$. With great interest in the catalytic logic and the mechanism for sulfur fate determination during 2,2'-bipyridine formation, in this study, we reconstituted the assembly line for the dethiolated antibiotics CAEs, validated the generality of 2,2'-bipyridine formation in the biosynthesis of the thiolated antibiotics COLs and experimentally dissected the on-line process for 2,2'-bipyridine formation and differentiation. The findings reported here exemplify the complexity and variety of assembly line chemistry, which has long been an area of intense interest in the creation of structurally diverse nonribosomal peptides, polyketides and their hybrid molecules.

\section{RESULTS \& DISCUSSION}

\section{2,2'-Bipyridine assembly line functions with a trans flavoprotein}

We began by reconstituting the activities of the didomain NRPS CaeA1 (650-aa), the PKS-NRPS hybrid CaeA2 (2484-aa) and the single-module NRPS CaeA3 (1047-aa) in vitro (Fig. 2). These multifunctional enzymes were individually produced in an engineered Escherichia coli strain expressing Sfp ${ }^{26,27}$, a phosphopantetheinyl (Ppant) transferase (PPTase) from Bacillus subtilis, leading to the in vivo conversion of their PCP and ACP domains from inactive apo-form into active holo-form by the thiolation of each active-site L-serine residue with Ppant. The CaeA1, CaeA2 and CaeA3 proteins produced here were soluble (Supplementary Fig. 1), and believed to be active and able to sequentially incorporate the starter and extender units via thiolated PCP/ACP $S$-(amino)acylation for chain elongation (Supplementary Fig. 2a). However, no off-line products were observed during the incubation with the substrates picolinic acid, malonyl-S-CoA, L-cysteine and L-leucine in the presence 
of adenosine triphosphate (ATP, necessary for A domain activity) (Fig. 2a, i). The CAE-related assembly line likely functions with certain partner(s) that act(s) in trans.

Analyzing the cae cluster revealed two genes, caeB1 and caeA4 (Fig. 1a), which encode a flavindependent protein and a Type II thioesterase, respectively. Both genes are immediately downstream of caeA2 and caeA3 and are likely cotranscripted with these upstream assembly line-encoding genes. To determine whether $\mathrm{CaeB} 1$ and $\mathrm{CaeA} 4$ are functionally related, we expressed them in E. coli (Supplementary Fig. 1). Purified CaeB1, which appeared light yellow, was determined to bind oxidized flavin adenine dinucleotide (FAD) in a noncovalent manner based on absorbance spectrum analysis and protein heating followed by high-performance liquid chromatography with highresolution mass spectrometry (HPLC-HR-MS) (Supplementary Fig. 3). The combination of CaeB1 with CaeA1, CaeA2 and CaeA3 resulted in the CAE intermediate 2,2'-bipyridinyl-L-leucine (1), whose production however did not require CaeA4-associated, putative thioesterase activity (Fig. 2a). Consequently, the CAE NRPS-PKS assembly line requires the trans activity of flavoprotein CaeB1 in the assembly of picolinic acid, malonyl-S-CoA, L-cysteine and L-leucine. In the reaction mixture containing tris(2-carboxyethyl)phosphine (TCEP) as a reducing agent, we observed tris(2carboxyethyl)phosphine-sulfide (2, Fig. 2b), which was indicative of $\mathrm{H}_{2} \mathrm{~S}$ production. $\mathrm{H}_{2} \mathrm{~S}$ was then trapped using compound $\mathbf{1 9}$ as a probe in the TCEP-free reaction mixture $(\text { Fig. } 2 \mathbf{c})^{28}$, confirming the involvement of dethiolation in the production of $\mathbf{1}$.

The trans flavoprotein oxidatively processes L-cysteinyl on PCP 
Phylogenetically, CaeB1 is close to flavin-dependent dehydrogenases, particularly those acting on acyl thioester substrates (Supplementary Fig. 3d) $)^{29,30}$. This observation led to the hypothesis that CaeB1 oxidatively processes L-cysteinyl on PCP prior to its noncanonical incorporation into Ring A of CAEs. We thus focused on CaeA2 and analyzed its changes in PCP $S$-aminoacylation using a Bottom-Up protein MS strategy $\mathrm{y}^{31,32}$. For method establishment, thiolated CaeA2 was subjected to protease hydrolysis, and peptide mixtures were analyzed by nanoLC-MS/MS to map the sequence containing the Ppant-modified L-serine residue. Extensive attempts revealed SLGGDSIMGIQF 2042 VSR (the relevant L-serine is underlined), a 15-aa sequence generated from complete trypsin treatment and partial chymotrypsin digestion (Supplementary Fig. 4a). This sequence can be detected by MS, largely due to its C-terminal alkaline residue, L-arginine, which facilitates peptide ionization. To eliminate the remaining chymotrypsin cleavage site and retain L-arginine, we engineered CaeA2 by mutating F2042 to L-leucine, L-isoleucine and L-valine based on residue conservation analysis (Supplementary Fig. 2b). The activity of each variant, i.e., CaeA2 ${ }^{\mathrm{F} 2042 \mathrm{~L}}, \mathrm{CaeA} 2^{\mathrm{F} 2042 \mathrm{I}}$ or CaeA2 ${ }^{\mathrm{F} 2042 \mathrm{~V}}$, was then assayed by substituting for wild-type CaeA2 in the 1-producing reaction mixture. In contrast to the mutations F2042I and F2042V, both of which caused a significant decrease in 1 production, F2042L had little effect on CaeA2 activity (Supplementary Fig. 2b). The CaeA2 ${ }^{\mathrm{F} 2042 \mathrm{~L}}$ variant can be completely digested using trypsin and chymotrypsin to efficiently produce SLGGDSIMGIQL2042VSR, facilitating analysis by HR-MS/MS to confirm the PCP sequence identity and covalently tethered substrate/intermediate (Supplementary Fig. 4b).

PCP S-aminoacylation-caused mass changes in SLGGDSIMGIQL2042VSR were then monitored (Fig. 3). The incubation of thiolated $\mathrm{CaeA} 2^{\mathrm{F} 2042 \mathrm{~L}}$ with L-cysteine yielded L-cysteinyl-S-CaeA2 $2^{\mathrm{F} 2042 \mathrm{~L}}$ 
(Supplementary Fig. 5a), consistent with the activities of the A and PCP domains of the CaeA2 NRPS module. The presence of L-cysteinyl in CaeA2 $2^{\mathrm{F} 2042 \mathrm{~L}}$ was confirmed by treatment with iodoacetamide (IAM, a thiol derivatization reagent), which yielded derivative 4 (with an MS signal 30-fold stronger than that of 3, Fig. 3 (ii, left) and Supplementary Fig. 5b). Incubation of the above mixture with CaeB1 significantly lowered the production of 3 by $\sim 70 \%$ (Fig. 3, iii, left). Accordingly, treatment with IAM generated 5, a derivative of (3-sulfhydryl)-pyruvoyl-S-CaeA2 $2^{\mathrm{F} 2042 \mathrm{~L}}$ (6) (Fig. 3 (iii, middle) and Supplementary Fig. 6a), indicating that CaeB1 acts on PCP for L-cysteinyl transformation. This conclusion was supported by the observation of a $+3 \mathrm{Da}$ derivative of $\mathbf{5}$ when $\mathrm{L}-[1,2,3-$ $\left.{ }^{13} \mathrm{C}_{3},{ }^{15} \mathrm{~N}\right]$ cysteine was used (Supplementary Fig. 6). Using L-[2,3,3-D 3 cysteine as the substrate resulted in deuterium-unlabeled 5 (Supplementary Fig. 6), consistent with the notion that CaeB1 catalyzes the $\alpha, \beta$-dehydrogenation of L-cysteinyl and converts 3 to dehydrocysteinyl-S-CaeA2 $2^{\mathrm{F} 2042 \mathrm{~L}}$ (7), which appears to be unstable and readily undergoes epimerization followed by hydrolysis to produce 6. Proton exchange can occur at the $\mathrm{C} \beta$ position of $\mathbf{6}$, leading to a loss of deuterium.

The sequence alignment of CaeB1 with various flavin-dependent dehydrogenases revealed potential key residues including E372 at the active site and S168 related to FAD binding (Supplementary Fig. 3d). These residues were mutated, generating $\mathrm{CaeB} 1^{\mathrm{E} 372 \mathrm{~L}}$ and $\mathrm{CaeB} 1^{\mathrm{S} 168 \mathrm{~A}}$. The former variant shares with the wild-type protein a light yellow color and an absorbance spectrum characteristic of FAD in oxidized form; in contrast, the latter variant was nearly colorless, indicating the loss of FAD-binding ability (Supplementary Fig. 3). CaeB1 $1^{\mathrm{E} 372 \mathrm{~L}}$ and $\mathrm{CaeB} 1^{\mathrm{S} 168 \mathrm{~A}}$ were individually used to replace wildtype $\mathrm{CaeB} 1$ in the 1-producing reaction mixture. Both mutations abolished $\mathbf{1}$ production, and saturation with an excess of FAD compensated the mutation S168A only (Supplementary Fig. 3). These results 
ascertained that $\mathrm{CaeB} 1$ activity is FAD-dependent. Mechanistically, CaeB1 catalyzes $\alpha, \beta-$ dehydrogenation likely utilizing E372 as a base for L-cysteinyl $\alpha$-deprotonation, followed by the elimination of a $\beta$-hydride equivalent to $\mathrm{FAD}$ to yield dehydrocysteinyl and $\mathrm{FADH}_{2}$ (Supplementary Fig. 7).

\section{The atypical NRPS Ct domain recruits trans flavoprotein activity}

Notably, CaeB1 activity strictly depends on the Ct domain of CaeA2, a C-like domain that is shortened by $\sim 1 / 3$ and lacks the catalytic L-histidine residue (Supplementary Fig. 8). Deleting this domain completely abolished the production of $\mathbf{1}$ (Fig. 2a, v). In the incubation of truncated CaeA2 $2^{\mathrm{F} 2042 \mathrm{~L}} \triangle \mathrm{Ct}$ with L-cysteine, L-cysteinyl-S-CaeA2 $2^{\mathrm{F} 2042 \mathrm{~L}} \triangle \mathrm{Ct}(\mathbf{8})$ was observed; however, the $\alpha, \beta$-dehydrogenation of L-cysteinyl failed to occur when CaeB1 was introduced (Fig. 3 (iv, left and middle) and Supplementary Fig. 9). We thus proposed that resembling the X domain of the NRPSs involved in glycopeptide biosynthesis ${ }^{33}, \mathrm{Ct}$ is necessary for recruiting the activity of the flavoprotein CaeB1 that functions in trans. To validate this hypothesis, we expressed the N-terminally thioredoxin (Trx)-tagged Ct domain in E. coli and measured its interaction with CaeB1 by isothermal titration calorimetry (ITC). Because CaeB1 is relatively unstable and tends to be precipitated during the measurement, we prepared the variant of this flavoprotein by fusion with maltose binding protein (MBP) at the N-terminus. ITC analysis revealed a $K_{\mathrm{d}}$ value of $0.53 \pm 0.12 \mu \mathrm{M}$ between MBP-fused CaeB1 variant and Trx-tagged $\mathrm{Ct}$ domain, indicating the existence of a moderate interaction (Fig. 4). In contrast, this interaction was not observed by titrating MBP-fused CaeB1 variant to control proteins, e.g., CaeA2 $\triangle \mathrm{Ct}$ (Supplementary Fig. 10a). Consequently, the flavoprotein CaeB1 likely functions in trans through the interaction with 
the $\mathrm{Ct}$ domain of CaeA2, leaving the Cy domain in the atypical NRPS module as the catalyst for dehydrocysteinyl extension and subsequent heterocyclization.

\section{2,2'-Bipyridine formation proceeds in the atypical NRPS module}

We then examined the elongating intermediate covalently tethered to the PCP domain of CaeA2 during 1 formation. The incubation of $\mathrm{CaeA} 1, \mathrm{CaeA} 2^{\mathrm{F} 2042 \mathrm{~L}}$ and $\mathrm{CaeB} 1$ with picolinic acid, malonyl-S-CoA and L-cysteine led to the accumulation of 2,2'-bipyridinyl-S-CaeA2 ${ }^{\mathrm{F} 2042 \mathrm{~L}}(\mathbf{9})$. In contrast, 9 was not observed in the presence of CaeA3 for 1 production (Fig. 3 (iv and v, right) and Supplementary Fig. 11a), supporting that 2,2'-bipyridinyl is an intermediate formed in the atypical NRPS module of CaeA2 before L-leucine extension. The atypical NRPS Cy domain likely catalyzes the formation of 2,2'bipyridinyl, because mutating the catalytic residue $\mathrm{D} 1165$ in its conserved motif $\mathrm{DxxxxD}(1165) \mathrm{xxS}$ to L-alanine completely abolished the production of $\mathbf{1}$ (Fig. 2a, vi). $\mathrm{H}_{2} \mathrm{~S}$ was observed during 9 formation (Fig. 2b), indicating that dethiolation occurred. The production of 2,2'-bipyridinyl in the atypical NRPS module of CaeA2 was further confirmed by HR-MS/MS analysis of the positive control of 2,2'bipyridinyl-S-CaeA2 $2^{\mathrm{F} 2042 \mathrm{~L}}$, which was generated by incubating unthiolated CaeA2 ${ }^{\mathrm{F} 2042 \mathrm{~L}}$ with synthetic 2,2'-bipyridinyl-S-CoA in the presence of Sfp (Fig. 3 (i, right) and Supplementary Fig. 11b). In addition, the combination of CaeA3 with truncated 2,2'-bipyridinyl-S-PCP $\mathrm{CaeA}_{2}$ (10, produced by thiolating the apo-form $\mathrm{PCP}_{\mathrm{CaeA} 2}$ domain with Sfp in the presence of 2,2'-bipyridinyl-S-CoA) and Lleucine produced 1 (Supplementary Fig. 12), validating that this single module NRPS accepts dethiolated 2,2'-bipyridinyl for L-leucine extension. Notably, 2,2'-bipyridinyl loses its chirality at C $\alpha$. This feature is consistent with the fact that CaeA3 possesses a ${ }^{D} C_{L}$ domain (Supplementary Fig. 13) ${ }^{34}$. 
A similar catalysis was observed in the biosynthesis of nocardicins ${ }^{35}$, in which an NRPS ${ }^{\mathrm{D}} \mathrm{C}_{\mathrm{L}}$ domain mediates the assembly of a $\mathrm{C} \alpha$-achiral intermediate with an L-amino acid for $\beta$-lactam formation.

\section{Timing of the dethiolation step in the formation of the CAE 2,2'-bipyridine core}

Focusing on the timing of the dethiolation step, we attempted to dissect the specific 2,2'-bipyridineforming process in the CAE assembly line (Fig. 5). Omitting CaeA1 for picolinic acid incorporation from the $\mathbf{1}$ or $\mathbf{9}$-forming reaction mixture prevented $\mathrm{H}_{2} \mathrm{~S}$ production (Fig. $\mathbf{2 b}$ ), inconsistent with route $a$ in which condensation follows the dethiolation of dehydrocysteinyl to 2-amino-allyl-S-CaeA2 (11). In addition, derivatives of the possible intermediate 11 (e.g., pyruvoyl-S-CaeA2, which might arise from epimerization and hydrolysis in a manner similar to the conversion of 7 to 6 ) in this route were not observed based on careful HR-MS/MS analysis. Removing the substrate picolinic acid from the 1 or 9-forming reaction mixtures led to similar results, supporting the hypothesis that the condensation with dehydrocysteinyl precedes its dethiolation. The Cy domain of CaeA2 shares high sequence homology to the counterparts known for thiazoline formation (Supplementary Figs. 8 and 14), in which related Cy domains display dual activity for L-cysteine or L-serine extension by forming an amide bond first and then cyclization via the nucleophilic addition of the $-\mathrm{SH}$ or $-\mathrm{OH}$ side chain of the newly incorporated residue onto the preceding carbonyl group to yield a five-membered thiazoline or azoline ring after $\mathrm{H}_{2} \mathrm{O}$ elimination ${ }^{3,5}$. However, the $\mathrm{Cy}$ domain of CaeA2 appears to be functionally distinct because it does not utilize unprocessed L-cysteinyl for condensation (Figs. 2 and 3). This domain likely follows CaeB1 activity and condenses the resulting reactive dehydrocysteinyl unit with the upstream intermediate for $\mathrm{C}$ - $\mathrm{C}$ bond formation through unprecedented $\mathrm{C} \beta$-nucleophilic 
substitution, yielding picolinyl-acetyl-dehydrocysteinyl-S-CaeA2 (12, Fig. 5) before heterocyclization to form the six-membered pyridine ring. We then utilized $\mathrm{L}-\left[2,3,3-\mathrm{D}_{3}\right]$ cysteine in the $\mathbf{1}$-forming reaction mixture. This assay led to the production of the + 1Da derivative of 1 (Supplementary Fig. 15), which excludes route $b$ in which cyclization precedes dethiolation. As shown in this route, cyclization requires the epimerization of $\mathbf{1 2}$ to the enamine, which would eliminate the $C \beta$ deuterium of the dehydrocysteinyl residue and result in unlabeled $\mathbf{1}$. Instead, route $c$ is favored based on this observation because the dethiolation of $\mathbf{1 2}$ to picolinyl-acetyl-dehydroalanyl-S-CaeA2 (13) facilitates cyclization to afford 2,2'-bipyridinyl and retain the $C \beta$ deuterium (Fig. 5). For dethiolation, reduced $\mathrm{FADH}_{2}$ might serve as a hydride supplier and undergo oxidation to produce FAD for recycling, consistent with a net non-redox result from this process (Supplementary Fig. 7a).

\section{2,2'-Bipyridine assembly line provides both dethiolated and thiolated intermediates}

Intriguingly, although route $b$ is not favored in CAE biosynthesis, skipping the dethiolation step allows the condensation of 5-sulfhydryl-2,2'-bipyridinyl-S-CaeA2 (14) with L-leucine to produce 5sulfhydryl-2,2'-bipyridinyl-L-leucine (15), the proposed off-loading intermediate in the biosynthesis of COLs (Fig. 5). Distinct from CAEs, COLs are 2,2'-bipyridine antibiotics possessing a sulfurcontaining group (Fig. 1). In addition to ColA1a and ColA1b, ColA2 and ColA3, which share high sequence homology with the CAE-related modular synth(et)ases CaeA1, CaeA2 and CaeA3, respectively, the biosynthetic pathway of COLs involves ColB1, the homolog of the flavoprotein CaeB1 that also noncovalently binds a FAD cofactor (Supplementary Fig. 3), and thus are assumed to share a similar trans flavin-dependent NRPS-PKS assembly line for the formation of the different 
thiolated $2,2^{\prime}$-bipyridine core ${ }^{14,25}$. To determine the difference in 2,2'-bipyridine formation, we attempted to replace the CAE enzymes with their counterparts from the COL pathway in the 1producing reaction mixture. These counterparts did not include those for picolinyl priming (i.e., CaeA1 or ColA1a/ColA1b) and subsequent two-carbon elongation (i.e., the PKS module of CaeA2 or ColA2), which are believed to be identical in the biosynthesis of CAEs or COLs. The trans partners ColB1 and CaeB1 were confirmed to be interchangeable in the production of $\mathbf{1}$ (Fig. 2a, vii), supporting that they both share the trans flavin-dependent activity for oxidatively processing L-cysteinyl to dehydrocysteinyl on PCP. Consistent with this finding, ITC analysis showed that ColB1 can interact with the $\mathrm{Ct}$ domain of the CaeA2, with a moderate $K_{\mathrm{d}}$ value of $1.18 \pm 0.15 \mu \mathrm{M}$ (Fig. 4). Interestingly, changing the NRPS CaeA3 to ColA3 completely abolished 1 production (Fig. 2a, viii), indicating that ColA3 differs from CaeA3, even though they both mediate L-leucine extension.

In vitro testing the exchangeability between the PKS-NRPS hybrid proteins CaeA2 and ColA2 failed because ColA2 was highly resistant to various methods for soluble protein preparation. We thus turned to in vivo biochemical assays and examined this exchangeability in the COL-producing strain Streptomyces roseosporus (Fig. 6). A chimeric gene that encodes the variant harboring the PKS module of ColA2 and the NRPS module of CaeA2, colA2 $2_{P K S-C a e A 2} 2_{N R S}$, was constructed and introduced into the previously developed, $\triangle$ colA2 mutant $S$. roseosporus strain that is incapable of producing COLs ${ }^{14}$. Remarkably, this complementation restored the production of COLs, supporting that the atypical NRPS modules of CaeA2 and ColA2 are functionally identical in terms of L-cysteinyl incorporation, trans CaeB1/ColB1 activity recruiting, condensation and heterocyclization during COL biosynthesis. These findings demonstrate the generality of both COLs and CAEs in 2,2'-bipyridine formation and 
particularly suggest that dethiolation can be skipped in the atypical NRPS module of CaeA2 to produce the thiolated 2,2'-bipyridine intermediate 5-sulfhydryl-2,2'-bipyridinyl. Notably, no dethiolated CAElike products were observed in the $S$. roseosporus strain when colA2 was replaced with colA2 $2_{P K S}$ caeA2 $2_{N R P S}$ (Fig. 6). Most likely, the specificity of the L-leucine extension determines the fate of the sulfur as the substitution with the CaeA2 NRPS module does not change the product profile.

\section{L-Leucine extension specializes 2,2'-bipyridine differentiation}

Unlike 2,2'-bipyridinyl, sulfhydryl-2,2'-bipyridinyl formed in the assembly line is highly reactive and proved to be difficult for analysis by aforementioned HR-MS/MS approaches. To in vitro evidence this covalently tethered thiol intermediate, we attempted to examine the downstream off-loading intermediates by incubating CaeA1, CaeA2 and CaeB1 with ColA3 in the presence of the necessary substrates and ATP. Treating the reaction mixture with IAM revealed 17, the derivative of the expected product 5-sulfhydryl-2,2'-bipyridinyl-L-leucine (15) (Supplementary Fig. 16). To further validate the production of 15 , we added ColG2, a COL pathway-specific methyltransferase ${ }^{14,25}$, into this reaction mixture. After L-leucine extension, ColG2 is believed to catalyze the $S$-methylation of the highly reactive thiol of intermediate $\mathbf{1 5}$ to produce the less reactive, previously characterized intermediate 5methylmercapto-2,2'-bipyridinyl-L-leucine (16). Indeed, 16 was observed as the sole product (Fig. 2a, ix). During this in vitro conversion, the cycling of $\mathrm{FADH}_{2}$ to $\mathrm{FAD}$ can go through the formation of the oxidizing adduct FAD-4a-OOH, in agreement with a net oxidation occurring in sulfhydryl-2,2'bipyridine formation (Supplementary Fig. 7b). In contrast, the combination of ColG2 with the complete CAE assembly line (i.e., CaeA1, CaeA2 and CaeA3 as well as CaeB1) failed to produce $\mathbf{1 6}$ 
and had little effect on the production of $\mathbf{1}$ (Fig. 2a, x), indicating that $\mathbf{1 5}$ cannot be generated in the presence of CaeA3. Furthermore, we narrowed the specificity of the upstream intermediate selection by $\mathrm{CaeA} 3$ and ColA3 to their $\mathrm{C}$ domains by domain swapping. Extensive attempts pointed to active $\mathrm{CaeA} 3 \mathrm{CColA} 3 \mathrm{~A}-\mathrm{PCP}$, the chimeric protein composed of the $\mathrm{C}$ domain of CaeA3 and the A-PCP didomain from ColA3. This protein was confirmed to be functionally identical to CaeA3 in the production of $\mathbf{1}$, albeit with a lower yield ( $70 \%$ decrease) (Fig. 2a, xi). It cannot be used to replace ColA3 because 16 was not observed in the presence of ColG2. Clearly, the CAE and COL assembly lines are identical for 2,2'-bipyridine formation and can simultaneously provide the dethiolated and thiolated intermediates (9 and 14). The only exception is the C domain-conferred specificity in Lleucine extension, which specializes the pathway to the production of either CAEs or COLs.

\section{CONCLUSION}

We demonstrate a common paradigm for 2,2'-bipyridine formation, which features a NRPS/PKS hybrid assembly line for sequential incorporation of the substrates picolinic acid, malonyl-S-CoA, Lcysteine and L-leucine with the association of a trans FAD-dependent partner (Fig. 5). This assembly line was first reconstituted in vitro for the biosynthesis of the dethiolated 2,2'-bipyridine antibiotics CAEs; then, the generality of 2,2'-bipyridine formation was validated in the biosynthesis of the distinct thiolated 2,2'-bipyridine antibiotics COLs, where the dethiolation step is skipped. In this assembly line, the atypical NRPS module Cy-A-PCP-Ct plays a central role in 2,2'-bipyridine formation. Following the A domain activity of this module for L-cysteine incorporation, the $\mathrm{Ct}$ domain recruits the activity of a flavoprotein that functions in trans for oxidatively processing L-cysteinyl on PCP. The Cy domain, 
which differs from the counterparts well-known for thiazoline formation, then condenses the resulting reactive dehydrocysteinyl extender unit with the upstream picolinyl-acetyl intermediate to afford a C$\mathrm{C}$ bond via unusual $\mathrm{C} \beta$-nucleophilic substitution, followed by intramolecular heterocyclization of the linear picolinyl-acetyl-dehydrocysteinyl intermediate to form the 2,2'-bipyridine core. Surprisingly, dethiolation can proceed or not between the condensation and heterocyclization steps, allowing for the production of both dethiolated and thiolated 2,2'-bipyridine intermediates in the atypical NRPS module. Subsequent L-leucine extension, which does not contribute any atoms in the final molecules, determines the sulfur fate and thus differentiates the biosynthesis of CAEs and COLs. Specifically, the C domain of the NRPS module C-A-PCP confers this selectivity for L-leucine extension and advances one of the two intermediates down a path to the 2,2'-bipyridine antibiotics with or without sulfur decoration. Therefore, whether dethiolation proceeds or is skipped appears to be not specifically controlled (i.e., not the key step) in the 2,2'-bipyridine assembly line because advancing the either intermediates (dethiolated or thiolated) strictly depends on the subsequent C domain selectivity for Lleucine extension. Based on this study, we provide a unique flavin-dependent paradigm in the assembly line, where cyclization reactions are usually difficult to be approached and thus remains poorly understood compared to those occurring at the off-line stage ${ }^{3,4}$. In addition, this study advances the understanding of the versatile functions of $\mathrm{C}$ domains in NRPS catalysis ${ }^{36}$. These domains include the gatekeeping $\mathrm{C}$ domain for sulfur fate determination, the $\mathrm{Ct}$ domain for trans flavin activity recruiting and, particularly, the unprecedented $\mathrm{Cy}$ domain for $\mathrm{C}-\mathrm{C}$ bond formation and heterocyclization. The findings reported here further our appreciation of assembly line enzymology and will facilitate the design, development and utilization of enzymatic molecular machinery to address synthetic challenges arising from structurally-related complex polypeptides, polyketides and their hybrids. 


\section{REFERENCES AND NOTES:}

1. Walsh, C. T. Polyketide and nonribosomal peptide antibiotics: modularity and versatility. Science 303, 1805-1810 (2004).

2. Walsh, C. T. \& Fischbach, M. A. Natural products version 2.0: connecting genes to molecules. $J$. Am. Chem. Soc. 132, 2469-2493 (2010).

3. Sundaram, S. \& Hertweck, C. On-line enzymatic tailoring of polyketides and peptides in thiotemplate systems. Curr. Opin. Chem. Biol. 31, 82-94 (2016).

4. Brown, A. S., Calcott, M. J., Owen, J. G. \& Ackerley, D. F. Structural, functional and evolutionary perspectives on effective re-engineering of non-ribosomal peptide synthetase assembly lines. Nat. Prod. Rep. 35, 1210-1228 (2018).

5. Pang, B., Wang, M. \& Liu, W. Cyclization of polyketides and non-ribosomal peptides on and off their assembly lines. Nat. Prod. Rep. 33, 162-173 (2016).

6. Nivina, A., Yuet, K. P., Hsu, J. \& Khosla, C. Evolution and Diversity of Assembly-Line Polyketide Synthases. Chem. Rev. 119, 12524-12547 (2019).

7. Kaes, C., Katz, A. \& Hosseini, M. W. Bipyridine: The Most Widely Used Ligand. A Review of Molecules Comprising at Least Two 2,2‘-Bipyridine Units. Chem. Rev. 100, 3553-3590 (2000).

8. Hapke, M., Brandt, L. \& Lutzen, A. Versatile tools in the construction of substituted 2,2'bipyridines-cross-coupling reactions with tin, zinc and boron compounds. Chem. Soc. Rev. 37, 2782-2797 (2008).

9. Funk, A. \& Divekar, P. V. Caerulomycin, a New Antibiotic from Streptomyces Caeruleus Baldacci: I. Production, Isolation, Assay, and Biological Properties. Can. J. Microbiol. 5, 317-321 (1959).

10. Gurram, R. K., Kujur, W., Maurya, S. K. \& Agrewala, J. N. Caerulomycin A enhances transforming growth factor-beta (TGF-beta)-Smad3 protein signaling by suppressing interferongamma (IFN-gamma)-signal transducer and activator of transcription 1 (STAT1) protein signaling to expand regulatory T cells (Tregs). J. Biol. Chem. 289, 17515-17528 (2014).

11. Singla, A. K. et al. Cerulomycin A: A Potent Novel Immunosuppressive Agent. Transplantation 97, E57-E59 (2014). 
12. Kaur, S., Srivastava, G., Sharma, A. N. \& Jolly, R. S. Novel immunosuppressive agent caerulomycin A exerts its effect by depleting cellular iron content. Br. J. Pharmacol. 172, 2286$2299(2015)$.

13. Kujur, W., Gurram, R. K., Haleem, N., Maurya, S. K. \& Agrewala, J. N. Caerulomycin A inhibits Th2 cell activity: a possible role in the management of asthma. Sci. Rep. 5, 15396-15405 (2015).

14. Qu, X. et al. Caerulomycins and collismycins share a common paradigm for 2,2'-bipyridine biosynthesis via an unusual hybrid polyketide-peptide assembly Logic. J. Am. Chem. Soc. 134, 9038-9041 (2012).

15. Zhu, Y. et al. Identification of caerulomycin A gene cluster implicates a tailoring amidohydrolase. Org. Lett. 14, 2666-2669 (2012).

16. Chen, D., Zhao, Q. \& Liu, W. Discovery of caerulomycin/collismycin-type 2,2'-bipyridine natural products in the genomic era. J. Ind. Microbiol. Biotechnol. 46, 459-468 (2019).

17. McInnes, A. G., Smith, D. G., Walter, J. A., Vining, L. C. \& Wright, J. L. C. The biosynthesis of caerulomycin A in Streptomycescaeruleus. Incorporation of 14C- and 13C-labeled precursors and analyses of labeling patterns by 13C nmr. Can. J. Chem. 57, 3200-3204 (1979).

18. Vining, L. C., McInnes, A. G., McCulloch, A. W., Smith, D. G. \& Walter, J. A. The biosynthesis of caerulomycins in Streptomyces caeruleus. Isolation of a new caerulomycin and incorporation of picolinic acid and glycerol into caerulomycin A. Can. J. Chem. 66, 191-194 (1988).

19. Zhu, Y. et al. Biochemical and Structural Insights into the Aminotransferase CrmG in Caerulomycin Biosynthesis. ACS Chem. Biol. 11, 943-952 (2016).

20. Zhu, Y. et al. Insights into caerulomycin A biosynthesis: a two-component monooxygenase CrmHcatalyzed oxime formation. J. Am. Chem. Soc. 135, 18750-18753 (2013).

21. Fu, P. et al. Acyclic congeners from Actinoalloteichus cyanogriseus provide insights into cyclic bipyridine glycoside formation. Org. Lett. 16, 4264-4267 (2014).

22. Chen, M. et al. Enzymatic Competition and Cooperation Branch the Caerulomycin Biosynthetic Pathway toward Different 2,2'-Bipyridine Members. Org. Biomol. Chem. 15, 5472-5475 (2017).

23. Chen, M., Pang, B., Du, Y. N., Zhang, Y. P. \& Liu, W. Characterization of the metallo-dependent amidohydrolases responsible for "auxiliary" leucinyl removal in the biosynthesis of 2,2'bipyridine antibiotics. Synth Syst Biotechnol 2, 137-146 (2017).

24. Shindo, K., Yamagishi, Y., Okada, Y. \& Kawai, H. Collismycins A and B, novel non-steroidal 
inhibitors of denamethasone-glucocorticoid receptor binding. J. Antibiot. 47, 1072-1074 (1994).

25. Garcia, I. et al. Elucidating the biosynthetic pathway for the polyketide-nonribosomal peptide collismycin A: mechanism for formation of the 2,2'-bipyridyl ring. Chem. Biol. 19, 399-413 (2012).

26. Quadri, L. E. et al. Characterization of Sfp, a Bacillus subtilis phosphopantetheinyl transferase for peptidyl carrier protein domains in peptide synthetases. Biochemistry 37, 1585-1595 (1998).

27. Pfeifer, B. A., Admiraal, S. J., Gramajo, H., Cane, D. E. \& Khosla, C. Biosynthesis of complex polyketides in a metabolically engineered strain of E. coli. Science 291, 1790-1792 (2001).

28. Wei, C. et al. Dual-Reactable Fluorescent Probes for Highly Selective and Sensitive Detection of Biological $\mathrm{H}_{2}$ S. Chem. Asian J. 11, 1376-1381 (2016).

29. Ghisla, S. \& Thorpe, C. Acyl-CoA dehydrogenases - A mechanistic overview. Eur. J. Biochem. 271, 494-508 (2004).

30. Watanabe, K., Khosla, C., Stroud, R. M. \& Tsai, S.-C. Crystal Structure of an Acyl-ACP Dehydrogenase from the FK520 Polyketide Biosynthetic Pathway: Insights into Extender Unit Biosynthesis. J. Mol. Biol. 334, 435-444 (2003).

31. Bumpus, S. B. \& Kelleher, N. L. Accessing natural product biosynthetic processes by mass spectrometry. Curr. Opin. Chem. Biol. 12, 475-482 (2008).

32. Gu, L. et al. GNAT-like strategy for polyketide chain initiation. Science 318, 970-974 (2007).

33. Haslinger, K., Peschke, M., Brieke, C., Maximowitsch, E. \& Cryle, M. J. X-domain of peptide synthetases recruits oxygenases crucial for glycopeptide biosynthesis. Nature 521, 105-109 (2015).

34. Rausch, C., Hoof, I., Weber, T., Wohlleben, W. \& Huson, D. H. Phylogenetic analysis of condensation domains in NRPS sheds light on their functional evolution. BMC Evol. Biol. 7, 7892 (2007).

35. Gaudelli, N. M., Long, D. H. \& Townsend, C. A. beta-Lactam formation by a non-ribosomal peptide synthetase during antibiotic biosynthesis. Nature 520, 383-387 (2015).

36. Bloudoff, K. \& Schmeing, T. M. Structural and functional aspects of the nonribosomal peptide synthetase condensation domain superfamily: discovery, dissection and diversity. BBA-Proteins and Proteomics 1865, 1587-1604 (2017). 
Supplementary Information is available in the online version of the paper.

Acknowledgements We thank Dr. L. Yi (Beijing University of Chemical Technology) and Prof. Z. Xi (Nankai University) for providing the fluorescent probe in $\mathrm{H}_{2} \mathrm{~S}$ examination. This work was supported in part by grants from NSFC (21750004, 21520102004, and 21621002), CAS (QYZDJ-SSW-SLH037 and XDB20020200), STCSM (17JC1405100), MST (2018ZX091711001-006-010) and K. C. Wang Education Foundation. 


\section{FIGURE LEGENDS}

Figure 1. Biogenesis of the polyketide-peptide hybrid, 2,2'-bipyridine antibiotics CAE-A and COLA. (a) Organization of the genes conserved in the CAE and COL biosynthetic gene clusters that codes for the hybrid NRPS/PKS assembly line and its associated trans FAD-dependent partner (gray) and other proteins (white). The didomain encoding gene caeAl in the cae cluster corresponds to the two discrete genes, colAla and colAlb, in the col cluster. (b) Hybrid NRPS/PKS assembly line for 2,2'bipyridine formation. The functional domains (AT and A domains) for substrate specificity are indicated in different colors with their associated building blocks: blue for picolinyl, yellow for malonyl, red for L-cysteinyl, and light gray for L-leucinyl. The sulfur atoms in the molecules are highlighted in red.

Figure 2. In vitro reconstitution of the 2,2'-bipyridine assembly line. (a) Analyses of 2,2'-bipyridine production by HPLC $(\lambda 315 \mathrm{~nm})$. The control reaction was conducted with the combination of the proteins CaeA1, CaeA2 and CaeA3 with the substrates picolinic acid, malonyl-S-CoA, L-cysteine, Lleucine, and ATP under aerobic conditions (i). To determine the trans partner, the reactions were conducted with $\mathrm{CaeB} 1$ (ii), $\mathrm{CaeA} 4$ (iii), or $\mathrm{CaeB} 1$ and CaeA4 (iv). To validate the necessity of $\mathrm{Ct}$, CaeA2 was replaced with CaeA2 $\triangle \mathrm{Ct}(\mathrm{v})$. To determine the catalytic role of the Cy domain of the atypical NRPS module, CaeA2 was replaced with CaeA2D1165A. (vi).To evaluate the component generality, the reactions were conducted with ColB1 (vii), CaeB1 and ColA3 (replacing CaeA3) (viii), CaeB1, ColA3 (replacing CaeA3) and ColG2 (ix), CaeB1 and ColG2 (x), or CaeB1 and CaeA3 ColA3 ${ }_{\text {A-PCP }}$ (replacing CaeA3) (x). Synthetic 1 (xii) and 16 (xiii) were used as standards. (b) Examination of $\mathrm{H}_{2} \mathrm{~S}$ production in TCEP-containing reaction mixtures by HPLC-MS. Electrospray 
ionization (ESI) $\mathrm{m} / z[\mathrm{M}+\mathrm{H}]^{+}$at 283.0405 (calcd.) for $\mathbf{2}$, which is generated by a reaction of $\mathrm{H}_{2} \mathrm{~S}$ with TCEP. The initial reaction was conducted by combining CaeA1, CaeA2, CaeA3 and CaeB1 with picolinic acid, malonyl-S-CoA, L-cysteine, L-leucine, and ATP (i), and reactions in the absence of CaeA3 (ii), picolinic acid and CaeA3 (iii) or CaeA1 and CaeA3 (iv) were also conducted. (C) Examination of $\mathrm{H}_{2} \mathrm{~S}$ production in TCEP-free reaction mixtures by HPLC with excitation at $370 \mathrm{~nm}$ and monitoring emission at $450 \mathrm{~nm}$. The reaction of the fluorescent probe $\mathbf{1 9}$ with $\mathrm{Na}_{2} \mathrm{~S}$ to yield $\mathbf{2 0}$ was used as the positive control (i). $\mathrm{H}_{2} \mathrm{~S}$ production was examined in the reactions where CaeA1, CaeA2, CaeA3 and CaeB1 were combined with picolinic acid, malonyl-S-CoA, L-cysteine and Lleucine in the presence (ii) and absence (iii) of ATP.

Figure 3. Examination of PCP-tethered intermediates during 2,2 '-bipyridine formation (top) by nanoLC-MS/MS (below). The sequence SLGGDSIMGIQL2042VSR (in the rectangle) arises from the complete digestion of CaeA2 $2^{\mathrm{F} 2042 \mathrm{~L}}$ using trypsin and chymotrypsin. For details of the HR-MS/MS analyses, see Figs. S4 S6, S9 and S11. The two IAM-treated sequences (ESI $m / z[\mathrm{M}+3 \mathrm{H}]^{3+}$ for the left, calcd. 678.3143; for the middle, calcd. 677.9704) come from L-cysteinyl-S-CaeA2 $2^{\mathrm{F} 2042 \mathrm{~L}}(\mathbf{3})$ and dehydrocysteinyl-S-CaeA2 $2^{\mathrm{F} 2042 \mathrm{~L}}$ (7), respectively, which were examined by incubating $\mathrm{CaeA} 2^{\mathrm{F} 2042 \mathrm{~L}}$ and L-cysteine in the absence (i, negative control) and presence (ii) of ATP, with CaeB1 (iii), CaeB1 and $\mathrm{CaeA} 2^{\mathrm{F} 2042 \mathrm{~L}} \triangle \mathrm{Ct}$ (replacing $\mathrm{CaeA} 2^{\mathrm{F} 2042 \mathrm{~L}}$ ) (iv), or CaeB1, CaeA1, picolinic acid and malonyl-SCoA (v). The right sequence (ESI $m / z[\mathrm{M}+\mathrm{H}]^{+}$, calcd. 690.9854) comes from 2,2'-bipyridinyl-S$\mathrm{CaeA} 2^{\mathrm{F} 2042 \mathrm{~L}}$ (9). The positive control was prepared by incubating CaeA2 ${ }^{\mathrm{F} 2042 \mathrm{~L}}$ (with PCP in apo form) and 2,2'-bipyridinyl-S-CoA (i). 9 was examined by incubating CaeA2 $2^{\mathrm{F} 2042 \mathrm{~L}}$ and L-cysteine (ii), with CaeB1 (iii), CaeB1, CaeA1, picolinic acid and malonyl-S-CoA (iv), or CaeB1, CaeA1, CaeA3, 
picolinic acid, malonyl-S-CoA and L-leucine (v).

Figure 4. Measurement of the interactions of the $\mathrm{Ct}$ domain of CaeA2 with related flavoproteins by ITC. Raw dates were shown on top, and the integrated curves containing experimental points and the best fitting line obtained from the single binding site model were shown on bottom. This measurement was conducted in triplicate. For negative controls, see Supplementary Fig. 10. (a) Titrating MBPfused CaeB1 to Trx-tagged Ct. (b) Titrating ColB1 to Trx-tagged Ct.

Figure 5. Proposed mechanisms for 2,2'-bipyridine formation. The PCP domain of CaeA2 is labeled in red. The $\mathrm{C}$ domains of CaeA3 and ColA3 are highlighted in green and blue, respectively. The favored pathways for CAEs and COLs are highlighted with light yellow and light purple backgrounds, respectively.

Figure 6. Domain organization of the NRPSs CaeA2, ColA2 and ColA2 ${ }_{\mathrm{PKS}-\mathrm{Cae}} 2_{\mathrm{NRPS}}$ (Top) and HPLC analysis of COL-related products in S. roseosporus strains (below). i, the wild-type COLproducing strain (positive control); ii, the $\triangle$ colA2 mutant in which a chimeric gene colA $_{P K S-c a e A 2_{N R P S}}$ is expressed in trans; and iii, the $\triangle$ colA2 mutant (negative control). 
Figure 1.

A

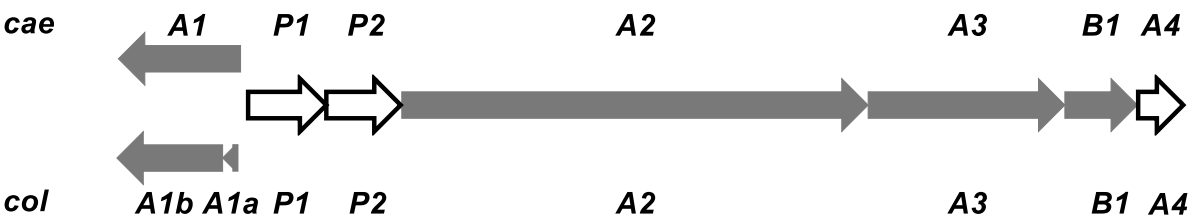

B

\section{CaeA1}<smiles>O=C(Sc1ccccc1)c1ccccc1</smiles><smiles>O=C(Sc1ccccc1)c1ccccn1</smiles>

(KS)

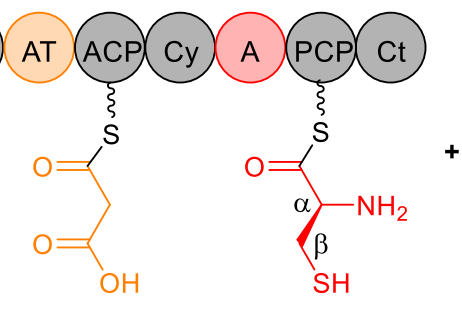

ColA2

\section{CaeA3}

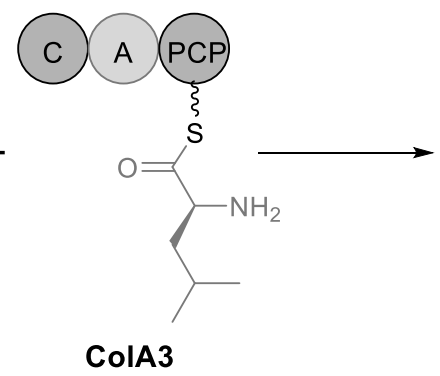

ColA1b ColA1a

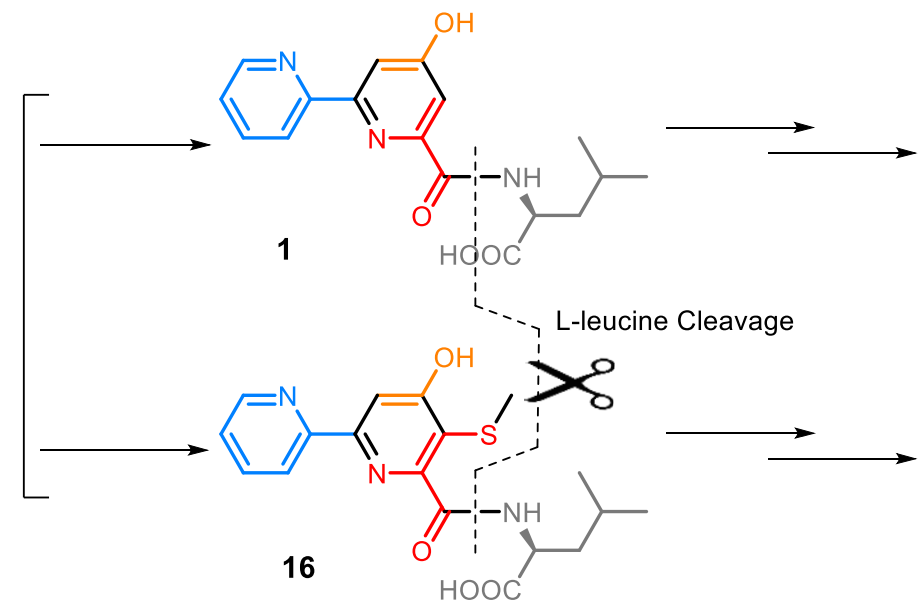

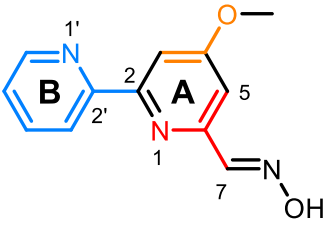

CAE-A

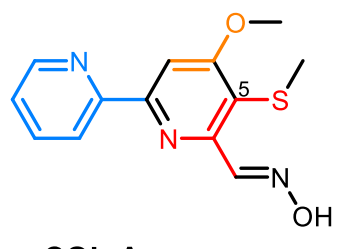


Figure 2.

a
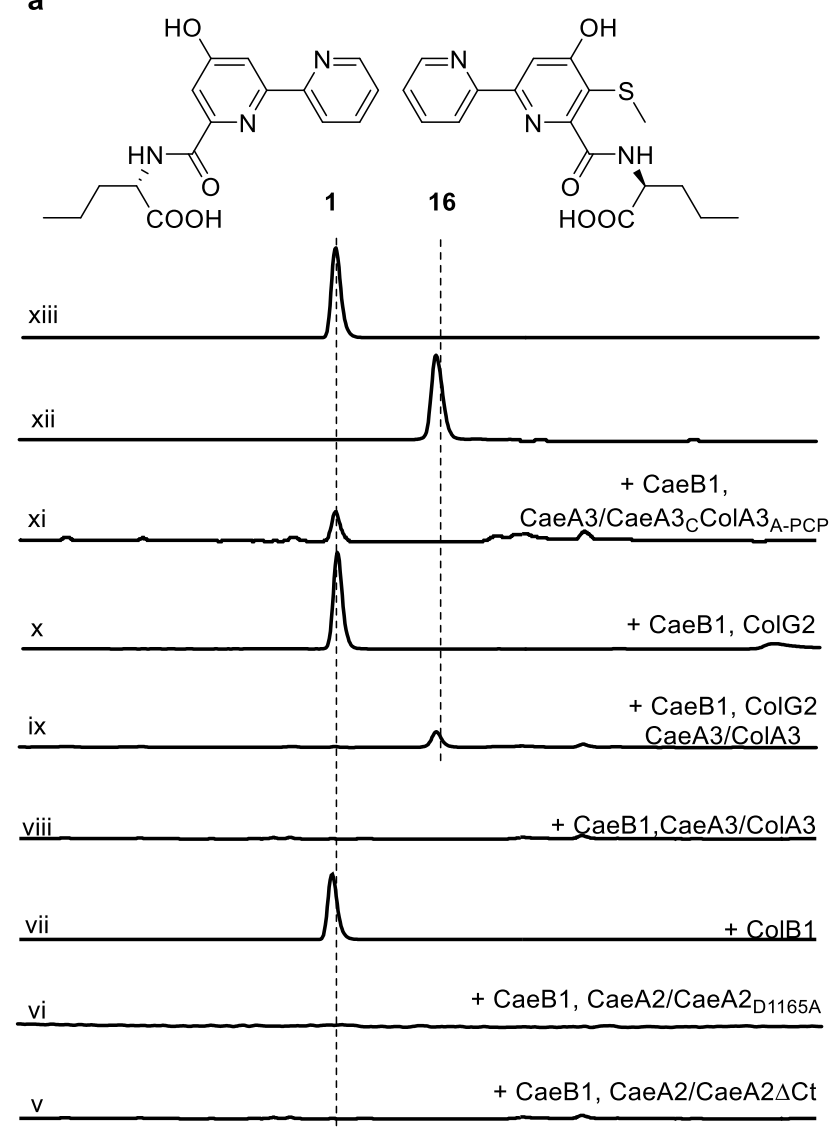

iv

+ CaeB1, CaeA4

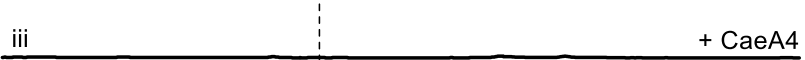

$\underline{\text { ii }}$

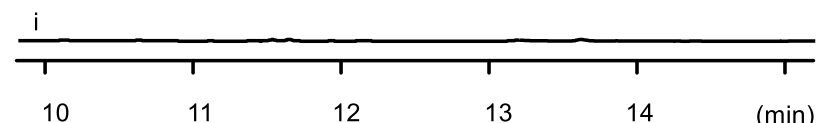

b<smiles>O=C(O)CCP(CCC(=O)O)CCC(=O)O</smiles>

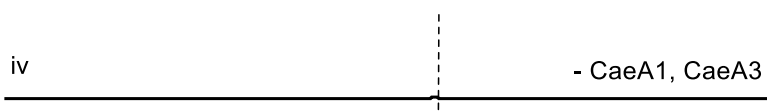

\begin{tabular}{l|l} 
iii & - CaeA3, pycolinic acid \\
\hline
\end{tabular}
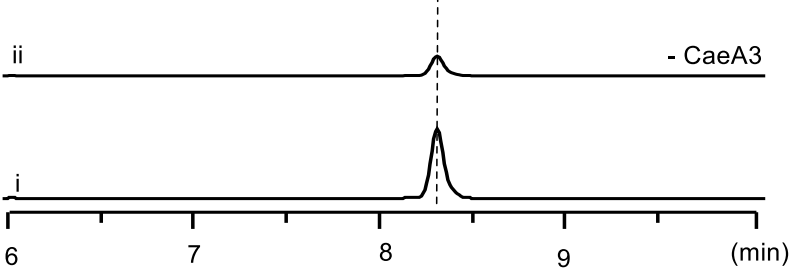

(1)

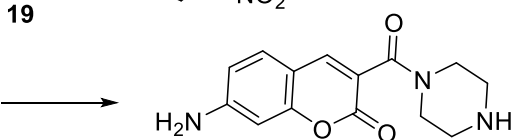

20
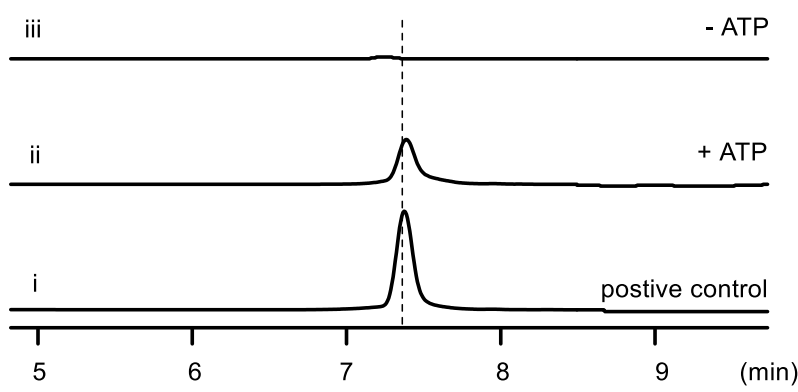
Figure 3.
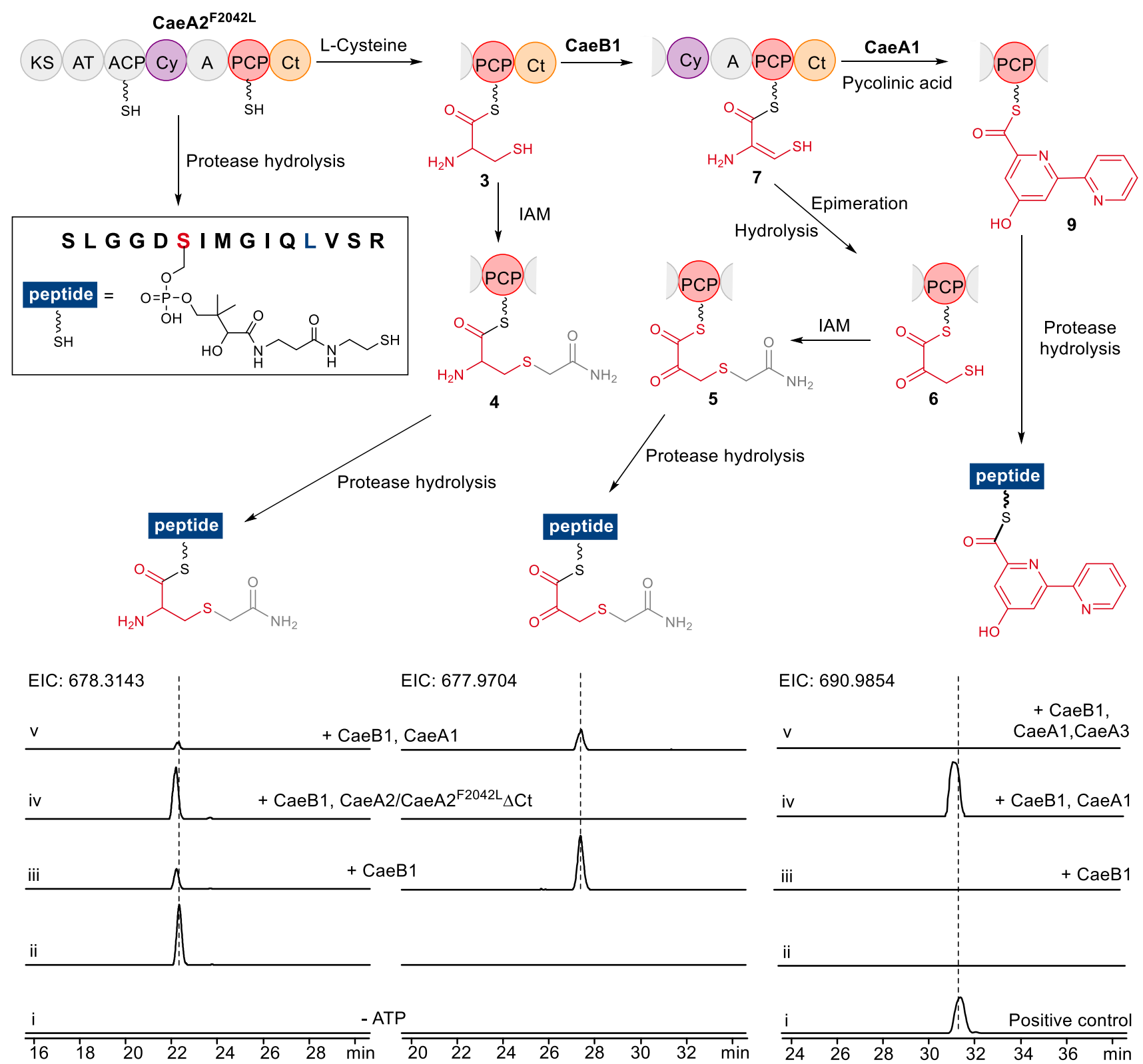
Figure 4.
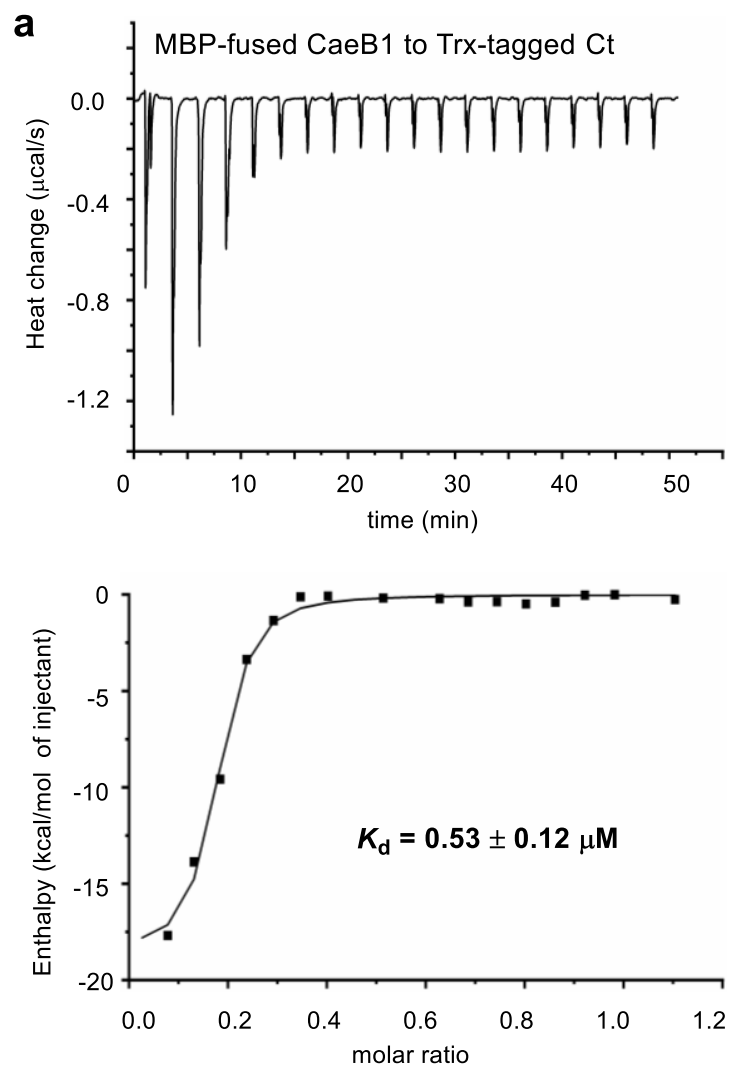
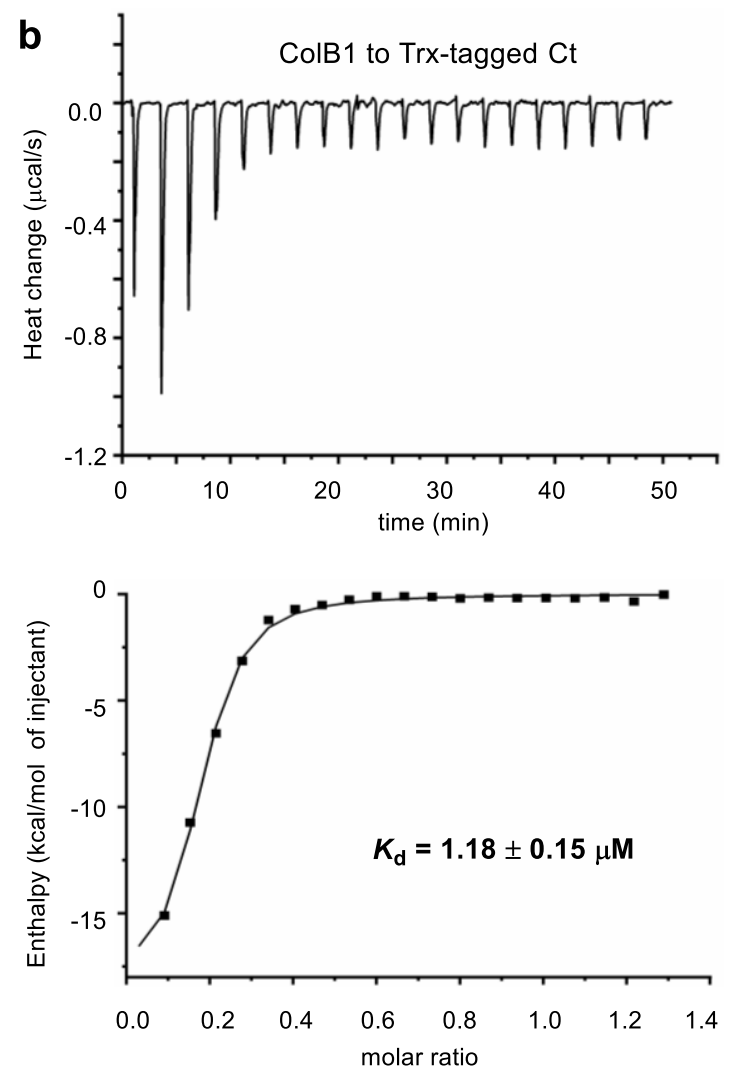


\section{Figure 5.}

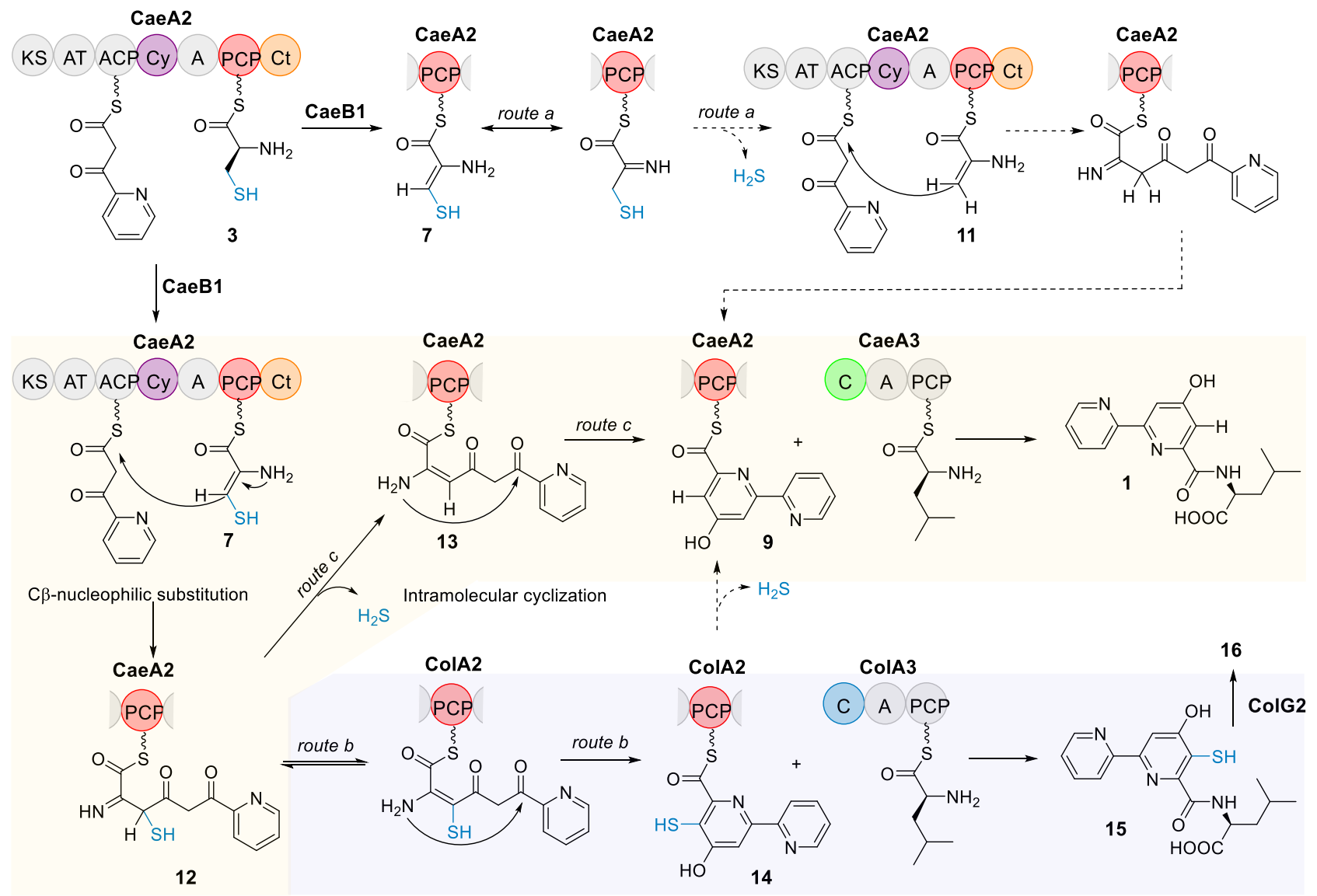


Figure 6.

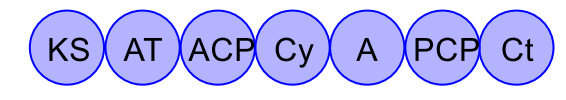

CaeA2

(KS) AT ACP Cy A PCP Ct

ColA2

(KS) AT ACP Cy A PCP Ct

CoIA2 $_{\text {PKS }}-$ CaeA2 $_{\text {NRPS }}$
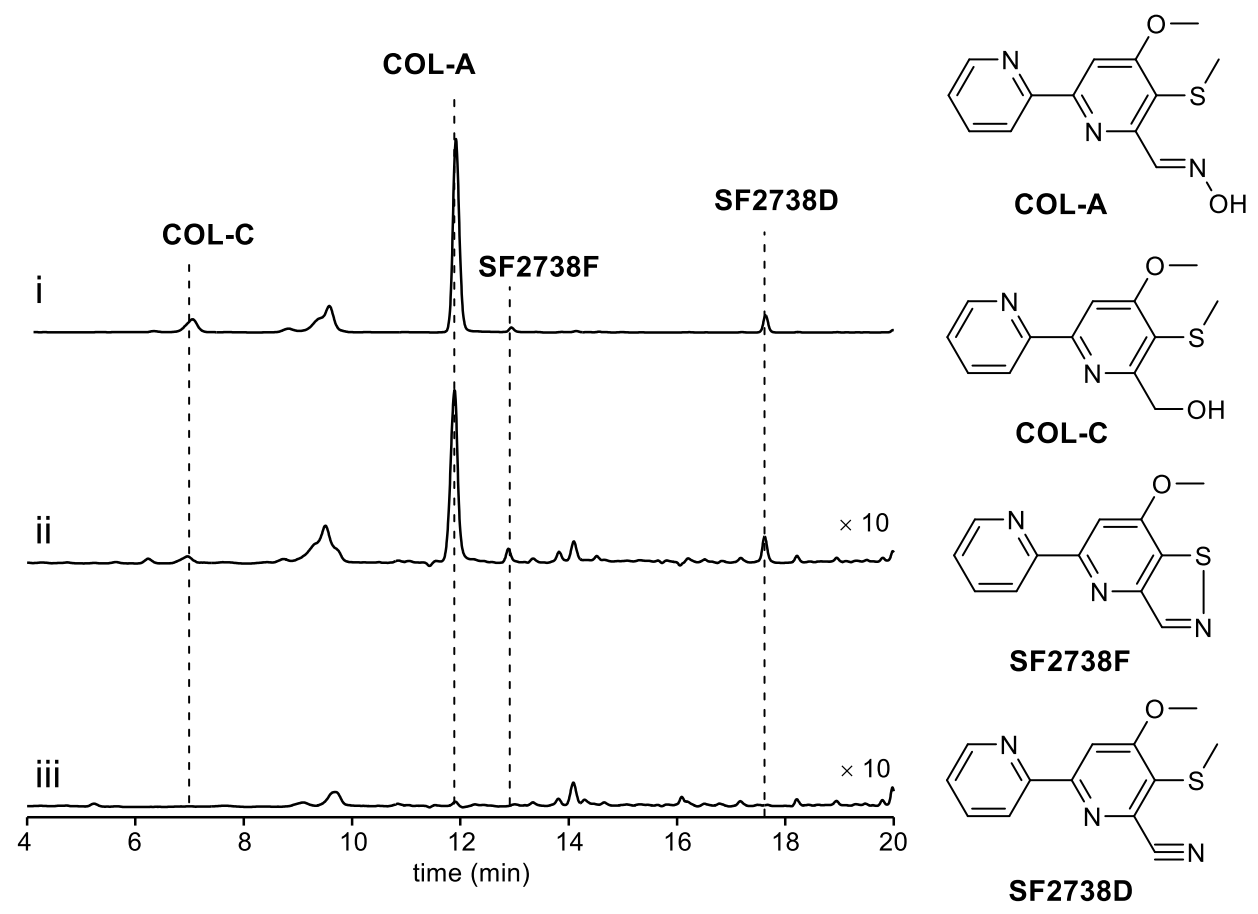


\section{Caerulomycin and Collismycin Antibiotics Share a trans Flavin-Dependent Assembly Line for}

\section{2,2'-Bipyridine Formation and Sulfur Fate Differentiation}

Bo Pang, ${ }^{1, \#}$ Rijing Liao, ${ }^{2, \#}$ Zhijun Tang, ${ }^{1 \#}$ Shengjie Guo, ${ }^{1}$ Zhuhua Wu, ${ }^{1}$ and Wen Liu ${ }^{1,3, *}$

${ }^{1}$ State Key Laboratory of Bioorganic and Natural Products Chemistry, Center for Excellence in

Molecular Synthesis, Shanghai Institute of Organic Chemistry, University of Chinese Academy of Sciences, 345 Lingling Road, Shanghai 200032, China;

${ }^{2}$ Shanghai Institute of Precision Medicine, Ninth People's Hospital, Shanghai Jiao Tong University School of Medicine, Shanghai 200125, China;

${ }^{3}$ Huzhou Center of Bio-Synthetic Innovation, 1366 Hongfeng Road, Huzhou 313000, China;

* To whom correspondence should be addressed: Shanghai Institute of Organic Chemistry, Chinese Academy of Sciences, 345 Lingling Rd., Shanghai 200032, China. Wen Liu, Email: wliu@sioc.ac.cn, Tel: 86-21-54925111, Fax: 86-21-64166128

\# These authors equally contribute to this work. 


\section{SUPPLIMENTARY METHODS}

General materials and methods. Biochemicals and media were purchased from Sinopharm Chemical Reagent Co., Ltd. (China), Oxoid Ltd. (U.K.) or Sigma-Aldrich Co. LLC. (USA) unless otherwise stated. Restriction endonucleases were purchased from Thermo Fisher Scientific Co. Ltd. (USA). Chemical reagents were purchased from standard commercial sources. The bacterial strains, plasmids and primers used in this study are summarized in Supplementary Tables 1, 2, and 3, respectively.

DNA isolation and manipulation in E. coli or actinobacteria were carried out according to standard methods ${ }^{1,2}$. PCR amplifications were carried out on an Applied Biosystems Veriti Thermal Cycler (Thermo Fisher Scientific Inc., USA) using either Taq DNA polymerase (Vazyme Biotech Co. Ltd, China) for routine verification or PrimeSTAR HS DNA polymerase (Takara Biotechnology Co., Ltd. Japan) for high fidelity amplification. Primer synthesis was performed at Shanghai Sangon Biotech Co. Ltd. (China). DNA sequencing was performed at Shanghai Majorbio Biotech Co. Ltd. (China).

High performance liquid chromatography (HPLC) analysis was carried out on an Agilent 1260 HPLC system (Agilent Technologies Inc., USA) equipped with a DAD detector. Semi-preparative HPLC was performed on an Agilent 1100 system equipped with a DAD detector (Agilent Technologies Inc., USA). HPLC Electrospray ionization MS (HPLC-ESI-MS) and tandem MS (MS/MS) for small molecules were performed on a Thermo Fisher LTQ Fleet ESI-MS spectrometer (Thermo Fisher Scientific Inc., USA), and the data were analyzed using Thermo Xcalibur software. NanoLC-MS/MS and MS/MS for peptides were performed on an EASY-nLC 1200 (Thermo Fisher Scientific Inc., USA) coupled with a 
Q Exactive HF mass spectrometer (Thermo Fisher Scientific Inc., USA), and the data were analyzed using pymzML, pFind ${ }^{3}$ and Thermo Xcalibur. High resolution ESI-MS (HR-ESI-MS) analysis for small molecules was carried out on an Agilent 6230B Accurate Mass TOF LC/MS System (Agilent Technologies Inc., USA), and the data were analyzed using Agilent MassHunter Qualitative Analysis software. NMR data were recorded on an Agilent $500 \mathrm{MHz}$ PremiumCompact+ NMR spectrometer (Agilent Technologies Inc., USA).

Protein expression and purification. The recombinant proteins $\mathrm{CaeA} 1, \mathrm{PCP}_{\mathrm{CaeA} 2}, \mathrm{CaeB} 1, \mathrm{ColB} 1$, ColG2, CaeA3, ColA3, and the chimeric NRPS protein CaeA3 ColA3 ${ }_{\mathrm{A}-\mathrm{PCP}}$, were produced in $\mathrm{N}$ terminal $6 \times$ His-tagged forms, while CaeA2 and its variants (e.g., CaeA2 ${ }^{\mathrm{F} 2042 \mathrm{~L}}$, CaeA2 ${ }^{\mathrm{F} 2042 \mathrm{I}}$, $\mathrm{CaeA} 2^{\mathrm{F} 2042 \mathrm{~V}}, \mathrm{Ct}_{\mathrm{CaeA} 2}, \mathrm{CaeA} 2-\triangle \mathrm{Ct}$ and $\mathrm{CaeA} 2^{\mathrm{F} 2042 \mathrm{~L}} \triangle \mathrm{Ct}$ ) were expressed in $\mathrm{C}$-terminal $8 \times$ His-tagged forms or N-terminal $6 \times$ His and Trx-tagged forms.

In general, the genes coding for the above proteins were amplified by PCR individually from the CAEproducing strain Actinoalloteichus cyanogriseus or the COL-producing strain Streptomyces roseosporus, both of which are listed in Supplementary Table 1, using the corresponding primers listed in Supplementary Table 3. The PCR products were first cloned into the vector pMD19-T for sequencing to confirm the fidelity and then into the expression vector $\mathrm{pET}-28 \mathrm{a}(+)$ (for N-terminal $6 \times$ His-tagged proteins) or pET-37b(+) (for C-terminal $8 \times$ His-tagged proteins). For expression of the chimeric NRPS protein $\mathrm{CaeA} 3{ }_{\mathrm{C}} \mathrm{ColA} 3{ }_{\mathrm{A}-\mathrm{PCP}}$ (containing the $\mathrm{C}$ domain of $\mathrm{CaeA} 3$ and the $\mathrm{A}$ and $\mathrm{PCP}$ domains of ColA3), a 1.4 kb DNA fragment amplified by PCR from A. cyanogriseus using the primers CaeA3-C-For and CaeA3-C-Rev was cloned into pMD19-T, yielding pQL1046. Similarly, a 1.8 kb 
DNA fragment amplified by PCR from S. roseosporus using the primers ColA3-APCP-For and ColA3APCP-Rev was cloned into pMD19-T, yielding pQL1047. The $1.4 \mathrm{~kb}$ NdeI-NheI fragment and the 1.8 $\mathrm{kb}$ NheI-HindIII fragment were recovered and co-ligated into the NdeI-HindIII site of pET-28a(+) to give pQL1048.

The resulting recombinant plasmids, which are listed in Supplementary Table 2, were transferred into E. coli BL21(DE3) for expression of CaeA2 ${ }^{\mathrm{F} 2042 \mathrm{~L}}, \mathrm{PCP}_{\mathrm{CaeA} 2}, \mathrm{Trx}-\mathrm{Ct}{ }_{\mathrm{CaeA}}$, CaeB1, ColG2 and ColB1 or into E. coli BAP1, an engineered strain capable of co-expressing the PPTase $\mathrm{Sfp}^{4}$, for expression of CaeA1, CaeA3, ColA3, and CaeA2 and its variants CaeA2 ${ }^{\mathrm{F} 2042 \mathrm{~L}}, \mathrm{CaeA} 2^{\mathrm{F} 2042 \mathrm{I}}, \mathrm{CaeA} 2^{\mathrm{F} 2042 \mathrm{~V}}, \mathrm{CaeA} 2-$ $\triangle \mathrm{Ct}, \mathrm{CaeA} 2^{\mathrm{F} 2042 \mathrm{~L}} \triangle \mathrm{Ct}$ and $\mathrm{CaeA} 3{ }_{\mathrm{C}} \mathrm{ColA} 3_{\mathrm{A}-\mathrm{PCP}}$.

The culture of each E. coli transformant was incubated in Luria-Bertani (LB) medium containing 50 $\mu \mathrm{g} / \mathrm{mL}$ kanamycin at $37^{\circ} \mathrm{C}$ and $220 \mathrm{rpm}$ until the cell density reached $0.6-0.8$ at $\mathrm{OD}_{600}$. Protein expression was induced by the addition of isopropyl- $\beta$-D-thiogalactopyranoside (IPTG) to the final concentration of $0.1 \mathrm{mM}$, followed by further incubation for $20 \mathrm{~h}$ at $25^{\circ} \mathrm{C}$. The cells were harvested by centrifuging at $5000 \mathrm{rpm}$ for $20 \mathrm{~min}$ at $4^{\circ} \mathrm{C}$ and were re-suspended in $30 \mathrm{~mL}$ of lysis buffer $(50 \mathrm{mM}$ Tris-HCl, 300 mM NaCl, 10\% glycerol, pH 7.8). After disruption by FB-110X Low Temperature Ultrapressure Continuous Flow Cell Disrupter (Shanghai Litu Mechanical Equipment Engineering Co., Ltd, China), the soluble fraction was collected and subjected to purification of each target protein using a HisTrap FF column (GE Healthcare, USA). The desired protein fractions, as determined by SDSPAGE, were concentrated and desalted using a PD-10 Desalting Column (GE Healthcare, USA) according to the manufacturer's protocols. The concentration of protein was determined by Bradford 
assay using bovine serum albumin (BSA) as the standard.

Sequence analysis. Open reading frames (ORFs) were identified using the FramePlot 4.0beta program (http://nocardia.nih.go.jp/fp4/). The deduced proteins were compared with other known proteins in the databases using available BLAST methods (http://blast.ncbi.nlm.nih.gov/Blast.cgi). Amino acid sequence alignments were performed using Vector NT1 and ESPript 3.0 (http://espript.ibcp.fr/ESPript/ESPript/).

\section{Chemical synthesis of 4-hydroxy-2,2'-bipyridine-6-carboxyloyl-S-CoA (2,2'-bipyidinyl-S-CoA).}

The precursor 4-hydroxy-2,2'-bipyridinyl-6-carboxylic acid hydrobromide (17) was synthesized according to the method described previously ${ }^{4}$.

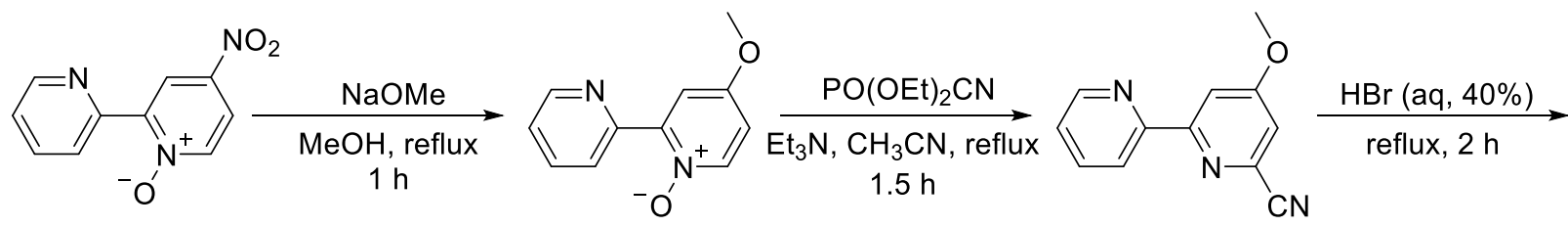

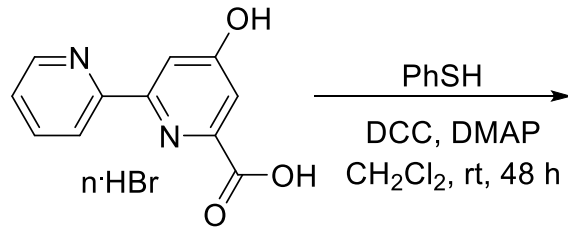

17<smiles>O=C(Sc1ccccc1)c1cc(O)cc(-c2ccccn2)n1</smiles>

18

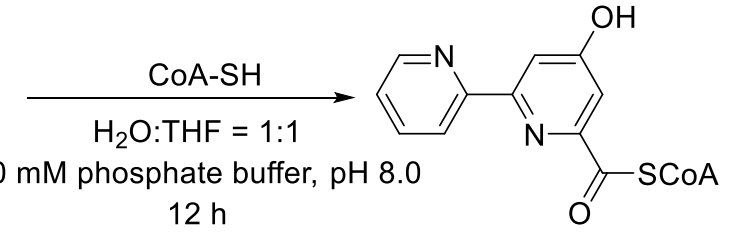

2,2'-bipyridinyl-S-CoA

4-Hydroxy-2,2'-bipyridinyl-6-carboxylic acid hydrobromide (415 mg) was suspended in $15 \mathrm{~mL}$ of anhydrous $\mathrm{CH}_{2} \mathrm{Cl}_{2}$. Then, $721 \mathrm{mg}$ of dicyclohexylcarbodiimide (DCC, $3.5 \mathrm{mmol}$ ), $0.58 \mathrm{~mL}$ of thiophenol (5.6 mmol) and $427 \mathrm{mg}$ of 4-dimethylaminopyridine (DMAP, $3.5 \mathrm{mmol}$ ) were added into 
this $\mathrm{CH}_{2} \mathrm{Cl}_{2}$ suspension stirred at $0^{\circ} \mathrm{C}$ under argon atmosphere. The mixture was stirred at room temperature for $48 \mathrm{~h}$ before adding $0.3 \mathrm{~mL}$ of $\mathrm{AcOH}$. After filtering, the organic phase of the reaction mixture was treated with saturated aqueous solution of $\mathrm{NaHCO}_{3}$ and brine, and subsequent concentrated to dryness under reduced pressure. The resulting residue was re-dissolved in EtOAc, washed with $5 \%(\mathrm{w} / \mathrm{v})$ citric acid solution, and filtered over diatomite. The organic phase was then dried over anhydrous $\mathrm{Na}_{2} \mathrm{SO}_{4}$ overnight, filtered, and concentrated under reduced pressure. Further purification was carried out by chromatography on silica gel using 2:1 $n$-hexane/EtOAc as the eluent, followed by concentration to yield $S$-phenyl 4-hydroxy-2,2'-bipyridinyl-6-carbothioate (18, $50.2 \mathrm{mg})$. for the purified compound, ${ }^{1} \mathrm{H}$ NMR $\left(500 \mathrm{MHz}, \mathrm{DMSO}-d_{6},\right) \delta 11.48(\mathrm{~s}, 1 \mathrm{H}), 8.72$ (ddd, $J=4.3,1.5$, $0.8 \mathrm{~Hz}, 1 \mathrm{H}), 8.49(\mathrm{~d}, J=7.9 \mathrm{~Hz}, 1 \mathrm{H}), 8.07(\mathrm{~d}, J=2.3 \mathrm{~Hz}, 1 \mathrm{H}), 8.04(\mathrm{td}, J=7.7,1.8 \mathrm{~Hz}, 1 \mathrm{H}), 7.57-$ $7.48(\mathrm{~m}, 6 \mathrm{H}), 7.30(\mathrm{~d}, J=2.3 \mathrm{~Hz}, 1 \mathrm{H})$; and ${ }^{13} \mathrm{C} \mathrm{NMR}\left(125 \mathrm{MHz}, \mathrm{DMSO}-d_{6}\right) \delta 191.7,166.9,157.1$, 154.2, 152.4, 149.9, 138.1, 135.3, 129.9, 129.7, 128.6, 125.3, 121.2, 112.1, 108.5. ESI-HRMS Calcd. for $\mathrm{C}_{17} \mathrm{H}_{13} \mathrm{~N}_{2} \mathrm{O}_{2} \mathrm{~S}^{+} 309.0698[\mathrm{M}+\mathrm{H}]^{+}$, found 309.0696.

Next, $30 \mathrm{mg}$ of CoA-SH (0.039 mmol, Sangon Biotech, China) was dissolved in $1.8 \mathrm{~mL}$ phosphate buffer $(40 \mathrm{mM}, \mathrm{pH}=8.0)$, and the $\mathrm{pH}$ was adjusted to 8.0 with $1 \mathrm{M} \mathrm{NaOH}$, followed by the addition of the solution of $18 \mathrm{mg}$ of the above obtained $S$-phenyl 4-hydroxy-2,2'-bipyridinyl-6-carbothioate in $1.8 \mathrm{~mL} \mathrm{THF}$, stirring at room temperature under argon atmosphere for $12 \mathrm{~h}$. After removal of THF under reduced pressure, the mixture was washed with ether (to remove the unreacted thioester), and then subjected to semi-preparation by HPLC to give 2,2'-bipyridinyl-S-CoA as a white powder (8 mg). This semi-preparation was carried out on an Aglient Zorbax column (SB-C18, $5 \mu \mathrm{m}, 9.4$ x $250 \mathrm{~mm}$, Agilent Technologies Inc., USA) by gradient elution of solvent $\mathrm{A}\left(\mathrm{H}_{2} \mathrm{O}\right.$ containing $\left.5 \mathrm{mM} \mathrm{NH} \mathrm{N}_{4} \mathrm{Ac}\right)$ and 
solvent $\mathrm{B}\left(\mathrm{CH}_{3} \mathrm{CN}\right)$ at a flow rate of $3 \mathrm{~mL} / \mathrm{min}$ over a 30 -min period as follows: $\mathrm{T}=0 \mathrm{~min}, 5 \% \mathrm{~B} ; \mathrm{T}=$ $2 \min , 5 \% \mathrm{~B} ; \mathrm{T}=20 \mathrm{~min}, 90 \% \mathrm{~B} ; \mathrm{T}=25 \mathrm{~min}, 90 \% \mathrm{~B} ; \mathrm{T}=26 \mathrm{~min}, 5 \% \mathrm{~B}$ and $\mathrm{T}=30 \mathrm{~min}, 5 \% \mathrm{~B}(\mathrm{mAU}$ at $254 \mathrm{~nm}$ ). For purified 2,2'-bipyridinyl-S-CoA, ESI-HRMS Calcd. for $\mathrm{C}_{17} \mathrm{H}_{13} \mathrm{~N}_{2} \mathrm{O}_{2} \mathrm{~S}^{+} 966.1682$ $[\mathrm{M}+\mathrm{H}]^{+}$, found 966.1670 .

The CAE 2,2'-bipyridine intermediate 1 and the COL 2,2'-bipyridine intermediate 16 were synthesized using the methods previously reported ${ }^{4}$.

Site-specific mutation of CaeA2 and its truncated proteins. Rolling cycle PCR amplification (using the primers listed in Supplementary Table 3) followed by subsequent DpnI digestion was conducted according to the standard procedure of the Mut Express II Fast Mutagenesis Kit (Vazyme Biotech Co. Ltd, China). The yielded recombinant plasmids were listed in Supplementary Table 2. Each mutation was confirmed by Sanger sequencing. CaeA2 $2^{\mathrm{F} 2042 \mathrm{~L}}, \mathrm{CaeA} 2^{\mathrm{F} 2042 \mathrm{~L}} \Delta \mathrm{Ct}$, CaeA2 $2^{\mathrm{F} 2042 \mathrm{I}}$, and $\mathrm{CaeA} 2^{\mathrm{F} 2042 \mathrm{~V}}$ were expressed in E.coli BAP1. All the mutant proteins were purified to homogeneity, and then concentrated according to the procedures for the native proteins described above.

Determination of the flavin cofactor. Each protein (CaeB1 or ColB1) solution at the concentration of $1 \mathrm{mg} / \mathrm{ml}$ was incubated at $100^{\circ} \mathrm{C}$ for $5 \mathrm{~min}$ for denaturation and then subjected to HPLC-DAD

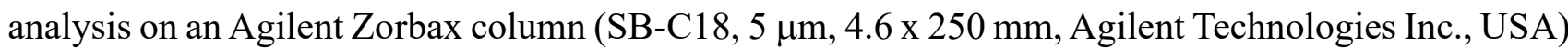
by gradient elution of solvent $\mathrm{A}\left(\mathrm{H}_{2} \mathrm{O}\right.$ containing $20 \mathrm{mM}$ ammonium acetate $)$ and solvent $\mathrm{B}\left(\mathrm{CH}_{3} \mathrm{CN}\right)$ at a flow rate of $1 \mathrm{~mL} / \mathrm{min}$ over a 35 -min period as follows: $\mathrm{T}=0 \mathrm{~min}, 5 \% \mathrm{~B} ; \mathrm{T}=2 \mathrm{~min}, 5 \% \mathrm{~B} ; \mathrm{T}=$ $20 \min , 90 \% \mathrm{~B} ; \mathrm{T}=25 \mathrm{~min}, 90 \% \mathrm{~B} ; \mathrm{T}=30 \mathrm{~min}, 5 \% \mathrm{~B}$ and $\mathrm{T}=35,5 \% \mathrm{~B}(\lambda$ at $448 \mathrm{~nm})$, using 
standard FAD as control. The supernatant of CaeB1 or ColB1 was subjected to ESI-HRMS analysis to confirmed the identity of FAD (ESI $m / z[\mathrm{M}+\mathrm{H}]^{+}$, calcd. 786.1644; found 786.1593).

Reconstitution of the 2,2'-bipyridine assembly line in vitro. The initial reaction was conducted at $30^{\circ} \mathrm{C}$ for $1 \mathrm{~h}$ in a $100 \mu \mathrm{L}$ reaction mixture containing $50 \mathrm{mM}$ Tris- $\mathrm{HCl}(\mathrm{pH} 7.5), 1 \mathrm{mM}$ TCEP, $10 \mathrm{mM}$ $\mathrm{MgCl}_{2}, 1 \mathrm{mM}$ picolinic acid, $1 \mathrm{mM}$ malonyl-CoA, $100 \mu \mathrm{M}$ L-cysteine, $1 \mathrm{mM}$ L-leucine, $10 \mu \mathrm{M} \mathrm{CaeA}$, $1 \mu \mathrm{M}$ CaeA2, $1 \mu \mathrm{M}$ CaeA3, and 4 mM ATP. To determine the necessary trans partner, CaeB1, CaeA4 or both of them were added into the above mixture, respectively, with the final concentration of $1 \mu \mathrm{M}$ for each protein. Each reaction was quenched with $100 \mu \mathrm{L}$ of $\mathrm{CH}_{3} \mathrm{CN}$ after incubation. To examine the production of the dethiolated 2,2'-bipyridine intermediate $\mathbf{1}$, reaction mixtures were subjected to HPLC analysis on an Agilent Zorbax column (SB-C18, $5 \mu \mathrm{m}, 4.6$ x 250 mm, Agilent Technologies Inc., USA) using a DAD detector, by gradient elution of solvent $\mathrm{A}\left(\mathrm{H}_{2} \mathrm{O}\right.$ containing $\left.0.1 \% \mathrm{TFA}\right)$ and solvent $\mathrm{B}\left(\mathrm{CH}_{3} \mathrm{CN}\right.$ containing $0.1 \%$ TFA $)$ at a flow rate of $1 \mathrm{~mL} / \mathrm{min}$ over a $35-\mathrm{min}$ period as follows: $\mathrm{T}=0 \min , 5 \% \mathrm{~B} ; \mathrm{T}=2 \min , 5 \% \mathrm{~B} ; \mathrm{T}=20 \mathrm{~min}, 90 \% \mathrm{~B} ; \mathrm{T}=25 \mathrm{~min}, 90 \% \mathrm{~B} ; \mathrm{T}=30 \mathrm{~min}, 5 \% \mathrm{~B}$ and $\mathrm{T}$ $=35,5 \% \mathrm{~B}$ ( $\lambda$ at $315 \mathrm{~nm}$ ). For HPLC-ESI-MS analysis, TFA was replaced by $0.1 \%$ formic acid.

To evaluate the participation of the enzymes and substrates in the production of $\mathbf{1}$, each component was omitted from the $100 \mu \mathrm{L}$ reaction mixture that contains $50 \mathrm{mM}$ Tris- $\mathrm{HCl}(\mathrm{pH} 7.5), 1 \mathrm{mM}$ TCEP, $10 \mathrm{mM} \mathrm{MgCl}$, $1 \mathrm{mM}$ picolinic acid, $1 \mathrm{mM}$ malonyl-CoA, $100 \mu \mathrm{M}$ L-cysteine, $1 \mathrm{mM}$ L-leucine, 10 $\mu \mathrm{M}$ CaeA1, $1 \mu \mathrm{M}$ CaeA2, $1 \mu \mathrm{M}$ CaeA3, $1 \mu \mathrm{M}$ CaeB1, and 4 mM ATP. To examine $\mathrm{O}_{2}$ dependence, gas exchange for $\mathrm{O}_{2}$ elimination was conducted in an anaerobic glovebox overnight before incubation under anaerobic conditions. To evaluate the mutation effects of CaeA2 on 1 production, CaeA2 was 
replaced with its variants $\mathrm{CaeA} 2^{\mathrm{F} 2042 \mathrm{~L}}, \mathrm{CaeA} 2^{\mathrm{F} 2042 \mathrm{I}}, \mathrm{CaeA} 2^{\mathrm{F} 2042 \mathrm{~V}}, \mathrm{CaeA} 2-\triangle \mathrm{Ct}$ or $\mathrm{CaeA} 2^{\mathrm{F} 2042 \mathrm{~L}} \triangle \mathrm{Ct}$. To mechanistically trace the transformation process, $\mathrm{L}-\left[1,2,3-{ }^{13} \mathrm{C}_{3},{ }^{15} \mathrm{~N}\right]$ cysteine and $\mathrm{L}-\left[2,3,3-\mathrm{D}_{3}\right]$ cysteine were used to replace unlabeled L-cysteine, respectively. To evaluate the changeability of the enzymes in the reaction mixture, 1) CaeB1 was replaced with ColB1 (with the final concentration of $10 \mu \mathrm{M}$ ); and 2) CaeA3 was replaced with ColA3 and CaeA3 3 ColA3 3 -PCP, respectively. Reactions are conducted at $30^{\circ} \mathrm{C}$ for $1 \mathrm{~h}$ and then quenched with $100 \mu \mathrm{L}$ of $\mathrm{CH}_{3} \mathrm{CN}$. The production of 1 was monitored by HPLC or HPLC-ESI-MS under conditions as described above.

For $\mathrm{H}_{2} \mathrm{~S}$ examination during 1 production, the reaction was conducted at $30^{\circ} \mathrm{C}$ for $1 \mathrm{~h}$ in the $100 \mu \mathrm{L}$, TCEP-involving reaction mixture that contains $50 \mathrm{mM}$ Tris- $\mathrm{HCl}\left(\mathrm{pH}\right.$ 7.5), $1 \mathrm{mM} \mathrm{TCEP,} 10 \mathrm{mM} \mathrm{MgCl}_{2}$, $1 \mathrm{mM}$ picolinic acid, $1 \mathrm{mM}$ malonyl-CoA, $100 \mu \mathrm{M}$ L-cysteine, $1 \mathrm{mM}$ L-leucine, $10 \mu \mathrm{M}$ CaeA1, $1 \mu \mathrm{M}$ CaeA2, $1 \mu \mathrm{M}$ CaeA3, $1 \mu \mathrm{M}$ CaeB1, and 4 mM ATP. Then, the TCEP derivative 2 was analyzed by HPLC-ESI-MS under conditions as described above. Alternatively, $\mathrm{H}_{2} \mathrm{~S}$ examination was conducted in the reaction mixture where TCEP was omitted, in the presence of $19(1 \mu \mathrm{M})$, a dual-reactable fluorescent probe used for highly selective and sensitive detection of biological $\mathrm{H}_{2} \mathrm{~S}^{5}$. The reaction of 19 with $\mathrm{Na}_{2} \mathrm{~S}$ to yield 20 serves as the control reaction. 20 was examined by HPLC-FLD under conditions as described above with excitation at $370 \mathrm{~nm}$ and relative emission at $450 \mathrm{~nm}$.

To examine whether the CAE assembly line provides the thiolated intermediate in COL biosynthesis, ColG2 (with the final concentration of $1 \mu \mathrm{M}$ ) was added into the $100 \mu \mathrm{L}$ reaction mixture that contains $50 \mathrm{mM}$ Tris-HCl (pH 7.5), $1 \mathrm{mM}$ TCEP, $10 \mathrm{mM} \mathrm{MgCl} 2,1 \mathrm{mM}$ picolinic acid, $1 \mathrm{mM}$ malonyl-CoA, $100 \mu \mathrm{M}$ L-cysteine, 1 mM L-leucine, $10 \mu \mathrm{M}$ CaeA1, $1 \mu \mathrm{M}$ CaeA2, $1 \mu \mathrm{M}$ CaeB1, $1 \mu \mathrm{M}$ CaeA3 (ColA3 
or $\mathrm{CaeA} 3 \mathrm{CColA} 3_{\mathrm{A}-\mathrm{PCP}}$ ), and $4 \mathrm{mM} \mathrm{ATP}$. The production of the thiolated 2,2'-bipyridine intermediate 16 was examined by HPLC or HPLC-ESI-MS under conditions as described above.

For sulfhydryl-2,2'-bipyridinyl-L-leucine (15) examination, the reaction was conducted at $30^{\circ} \mathrm{C}$ for 1 $\mathrm{h}$ in the $50 \mu \mathrm{L}$ reaction mixture contains $50 \mathrm{mM}$ Tris- $\mathrm{HCl}(\mathrm{pH} 7.5), 1 \mathrm{mM}$ TCEP, $10 \mathrm{mM} \mathrm{MgCl} 2,1$ $\mathrm{mM}$ picolinic acid, $1 \mathrm{mM}$ malonyl-CoA, $100 \mu \mathrm{M}$ L-cysteine, $1 \mathrm{mM}$ L-leucine, $10 \mu \mathrm{M}$ CaeA1, $1 \mu \mathrm{M}$ CaeA2, $1 \mu \mathrm{M}$ ColA3, $1 \mu \mathrm{M}$ CaeB1, and $4 \mathrm{mM}$ ATP. The reaction mixture was treated with 1\% SDS and $5 \mathrm{mM}$ TCEP at $55^{\circ} \mathrm{C}$ for $30 \mathrm{~min}$ to release the free thiol of 15 , and then was incubated with $25 \mathrm{mM}$ iodoacetamide (IAM) at $30^{\circ} \mathrm{C}$ for $30 \mathrm{~min}$. The production of 17 was examined by HPLC or HPLCESI-MS under conditions as described above.

In vitro assays of PCP $S$-aminoacylation on CaeA2 by nanoLC-MS/MS. The CaeA2 recombinant protein that was purified from E.coli BAP1 was subjected to complete or partial protease hydrolysis with trypsin, Glu-C or chymotrypsin as well as a variety of their combinations to map SLGGDSIMGIQF 2042VSR of CaeA2, the MS-detectable sequence that contains the Ppant-modified active-site L-serine residue (underlined). The digestion mixtures were filtrated using Microcon YM-10 (MilliporeSigma, USA) by centrifugation and stored at $-80^{\circ} \mathrm{C}$ before analysis. For nanoLC-MS/MS analysis, each sample was loaded on a trap column $(75 \mu \mathrm{m}$ i.d., $2 \mathrm{~cm}, \mathrm{C} 18,5 \mu \mathrm{m}, 100 \AA$, Thermo Fisher Scientific Inc., USA) for online desalting, and then was separated using a reversed phase column (75 $\mu \mathrm{m}$ i.d., $10.2 \mathrm{~cm}, \mathrm{C} 18,3 \mu \mathrm{m}, 120 \AA$, New Objective Inc., USA) by gradient elution of solvent A ( $\mathrm{H}_{2} \mathrm{O}$ containing $0.1 \%$ formic acid) and solvent $\mathrm{B}\left(80 \% \mathrm{CH}_{3} \mathrm{CN}\right.$ containing $0.1 \%$ formic acid) at a flow rate of $300 \mathrm{~nL} / \mathrm{min}$ over a $1.5 \mathrm{~h}$ period as follows: $\mathrm{T}=0 \mathrm{~min}, 18 \% \mathrm{~B} ; \mathrm{T}=45 \mathrm{~min}, 45 \% \mathrm{~B} ; \mathrm{T}=50 \mathrm{~min}$, 
$100 \% \mathrm{~B}$; and $\mathrm{T}=90 \mathrm{~min}, 100 \% \mathrm{~B}$. For MS analysis, the nano-ESI voltage and capillary temperature were set at $2.2 \mathrm{kV}$ and $275^{\circ} \mathrm{C}$, respectively. The MS data were acquired in data-dependent mode. Each full-scan MS ( $m / z$ 350-2000, resolution of $60 \mathrm{k})$ was followed with $10 \mathrm{HCD}$ MS/MS scans (normalized collision energy of 33, resolution of $15 \mathrm{k}$ ) for the most intense precursor ions. The maximum ion injection time for MS and MS/MS were 50 and $45 \mathrm{~ms}$, and the auto gain control target for MS and MS/MS were $3 \times 10^{6}$ and $5 \times 10^{4}$, respectively. The dynamic-exclusion time was set as 40 S.

To obtain the sequence SLGGDSIMGIQF ${ }_{2042} \mathbf{V S R}$, the $50 \mu \mathrm{L}$ solution that contains $50 \mathrm{mM}$ Tris- $\mathrm{HCl}$ (pH 7.5) and $5 \mu \mathrm{M}$ CaeA2 was treated with $4 \mu \mathrm{g}$ of trypsin (sequencing grade, Promega Corp., USA) at $30^{\circ} \mathrm{C}$ for $20 \mathrm{~min}$ (leading to complete digestion) and then with $0.6 \mu \mathrm{g}$ of chymotrypsin (sequencing grade, Promega Corp., USA) at $30^{\circ} \mathrm{C}$ for $10 \mathrm{~min}$ (leading to partial digestion that retains the $\mathrm{C}$-terminal sequence $\mathbf{F}_{2042}$ VSR). To obtain the engineered sequence SLGGDSIMGIQL2042VSR, the CaeA2 $2^{\mathrm{F} 2042 \mathrm{~L}}$ recombinant protein that was purified from E.coli BAP1 and its variants different in $S$ (amino)acylation underwent complete digestion with trypsin $(4 \mu \mathrm{g})$ and chymotrypsin $(0.6 \mu \mathrm{g})$ at $30^{\circ} \mathrm{C}$ for $20 \mathrm{~min}$.

To identify the target sequence that contains the Ppant-modified active-site L-serine residue, the raw MS data was processed using the pFind software by setting Ppant as a variable posttranslational modification. The selected sequence was then validated by HR-MS/MS analysis of the parent ion, its associated fragmented peptide ions and particularly the characteristic Ppant ejection ion ${ }^{6}$. 
To prepare L-cysteinyl-S-CaeA2 $2^{\mathrm{F} 2042 \mathrm{~L}}(\mathbf{3})$, the reaction was conducted at $30^{\circ} \mathrm{C}$ for $10 \mathrm{~min}$ in a $50 \mu \mathrm{L}$ reaction mixture containing $50 \mathrm{mM}$ Tris- $\mathrm{HCl}(\mathrm{pH} 7.5), 1 \mathrm{mM}$ TCEP, $10 \mathrm{mM} \mathrm{MgCl} 2,100 \mu \mathrm{M}$ Lcysteine, $5 \mu \mathrm{M} \mathrm{CaeA} 2^{\mathrm{F} 2042 \mathrm{~L}}$ (or CaeA2 ${ }^{\mathrm{F} 2042 \mathrm{~L}} \triangle \mathrm{Ct}$ ) and $4 \mathrm{mM}$ ATP. To prepare (3-sulfhydryl)-pyruvoyl$S$-CaeA2 $2^{\mathrm{F} 2042 \mathrm{~L}}(6)$, the reaction was conducted at $30^{\circ} \mathrm{C}$ for $10 \mathrm{~min}$ in a $50 \mu \mathrm{L}$ reaction mixture containing $50 \mathrm{mM}$ Tris- $\mathrm{HCl}(\mathrm{pH}$ 7.5), $1 \mathrm{mM}$ TCEP, $10 \mathrm{mM} \mathrm{MgCl} 2,100 \mu \mathrm{M}$ L-cysteine, $5 \mu \mathrm{M}$ $\mathrm{CaeA} 2^{\mathrm{F} 2042 \mathrm{~L}}$ (or $\mathrm{CaeA} 2^{\mathrm{F} 2042 \mathrm{~L}} \triangle \mathrm{Ct}$ ), $1 \mu \mathrm{M}$ CaeB1 and $4 \mathrm{mM}$ ATP. To prepare 2,2'-bipyridinyl-S$\mathrm{CaeA} 2^{\mathrm{F} 2042 \mathrm{~L}}(9)$, the reaction was conducted at $30^{\circ} \mathrm{C}$ for $10 \mathrm{~min}$ in a $50 \mu \mathrm{L}$ reaction mixture containing $50 \mathrm{mM}$ Tris- $\mathrm{HCl}(\mathrm{pH}$ 7.5), $1 \mathrm{mM}$ TCEP, $10 \mathrm{mM} \mathrm{MgCl} 2,1 \mathrm{mM}$ picolinic acid, $1 \mathrm{mM}$ malonyl-CoA, $100 \mu \mathrm{M}$ L-cysteine, $1 \mu \mathrm{M}$ CaeA1, $1 \mu \mathrm{M}$ CaeB1, $5 \mu \mathrm{M}$ CaeA2 $2^{\mathrm{F} 2042 \mathrm{~L}}$ (or CaeA2 $2^{\mathrm{F} 2042 \mathrm{~L}} \triangle \mathrm{Ct}$ ) and $4 \mathrm{mM}$ ATP. For isotope labeling, $\mathrm{L}-\left[1,2,3-{ }^{13} \mathrm{C}_{3},{ }^{15} \mathrm{~N}\right]$ cysteine and $\mathrm{L}-\left[2,3,3-\mathrm{D}_{3}\right]$ cysteine were used to replace unlabeled L-cysteine, respectively. For thiol derivatization, each reaction mixture was treated with 10 $\mathrm{mM}$ iodoacetamide (IAM) at $30^{\circ} \mathrm{C}$ for $2 \mathrm{~min}$. Protease digestion and subsequent nanoLC-MS/MS analyses were conducted using approaches as described above.

To probe potential intermediates, all MS/MS data of the derivatives from the sequence (SLGGDSIMGIQL2042VSR) were extracted from raw MS data using script written by Python (https://github.com/billpb610/AlFinder/blob/master/AlFinderS2.py). The script also recorded information about precursor ions, fragment iond and Ppant ejection ions for further analysis.

In vitro preparation of 2,2'-bipyridinyl-S-CaeA2 $2^{\mathrm{F2042L}}$ (9). To prepare 9 as a positive control in $S$ aminoacylation assays, the reaction was conducted at $30^{\circ} \mathrm{C}$ for $30 \mathrm{~min}$ in a $50 \mathrm{uL}$ reaction mixture containing 50 mM Tris-HCl (pH 7.5), 1 mM TCEP, 10 mM MgCl $2,100 \mu \mathrm{M}$ 2,2'-bipyridinyl-S-CoA, $5 \mu \mathrm{M}$ Sfp, and $10 \mu \mathrm{M}$ CaeA2 $2^{\mathrm{F} 2042 \mathrm{~L}}$ in apo-form. Protease digestion and subsequent nanoLC-MS/MS 
analyses were conducted using approaches as described above.

In vitro preparation of 2,2'-bipyridinyl-S-PCPCaeA2 (10). The reaction was conducted at $30^{\circ} \mathrm{C}$ for 1 $\mathrm{h}$ in an $80 \mu \mathrm{L}$ reaction mixture containing $62.5 \mathrm{mM}$ Tris- $\mathrm{HCl}(\mathrm{pH}=7.5), 1.25 \mathrm{mM}$ TCEP, $12.5 \mathrm{mM}$ $\mathrm{MgCl}_{2}, 1 \mathrm{mM}$ 2,2'-bipyridinyl-S-CoA, $100 \mu \mathrm{M}$ PCP $\mathrm{CaeA}_{2}$ (derived from wild-type CaeA2) in apo-form, and $4 \mu \mathrm{M}$ Sfp.

To produce the dethiolated 2,2'-bipyridine intermediate 1 , the reaction mixture was combined with the following components in a new $100 \mu \mathrm{L}$ reaction mixture: $1 \mathrm{mM}$ L-leucine, $5 \mu \mathrm{M}$ CaeA3, and $4 \mathrm{mM}$ ATP. After incubation at $30^{\circ} \mathrm{C}$ for $2 \mathrm{~h}$, the reaction was quenched with $100 \mu \mathrm{L}$ of $\mathrm{CH}_{3} \mathrm{CN}$ for $\mathrm{HPLC}$ or HPLC-ESI-MS analysis under conditions as described above.

Measurement of protein-protein interactions by isothermal titration calorimetry (ITC). ITC was performed with MicroCal-ITC200 (Malvern) at $25^{\circ} \mathrm{C}$. A $400 \mu 1$ aliquot of $60 \mu \mathrm{M} \mathrm{Ct}$ or CaeA2 $\triangle \mathrm{Ct}$ was placed in the stirred cell, and $120 \mu \mathrm{l}$ aliquot of $400 \mu \mathrm{M}$ MBP-fused CaeB1 or ColB1 was prepared in the syringe. All the recombinant proteins were prepared by Ni-affinity chromatography followed by size-exclusion chromatography (SEC) for purification, and then were exchanged in the $50 \mathrm{mM}$ Tris$\mathrm{HCl}(\mathrm{PH}=7.5)$ buffer containing $100 \mathrm{mM} \mathrm{NaCl}, 0.1 \mathrm{mM}$ TCEP and $5 \%$ glycerol. The titration was performed as follows: $1 \mu \mathrm{l}$ of protein in the syringe over $0.8 \mathrm{~s}$ for the first injection, followed by 19 injections of $2 \mu 1$ protein in the stirred cell at $120 \mathrm{~s}$ intervals. The heat of reaction per injection was determined by integration of the peak areas using the MicroCal-PEAQ-ITC software, which provides the best-fit values for the heat of binding $(\Delta \mathrm{H})$, the stoichiometry of binding $(\mathrm{N})$, and the dissociation constant $\left(K_{\mathrm{d}}\right)$. The heats of dilution were determined by injecting flavoprotein alone into the buffer and 
were subtracted from the corresponding experiments before curve fitting.

\section{Analysis of the functional exchangeability between CaeA2 and ColA2 in vivo. A $4.4 \mathrm{~kb}$ DNA}

fragment amplified by PCR from the CAE-producing $A$. cyanogriseus strain using the primers caeCO-for and cae-Ct-rev was cloned into pMD19-T, yielding pQL1053. After sequencing to confirm the fidelity, this PCR product was recovered by digestion with BgIII-EcoRV and then utilized to replace the $4.4 \mathrm{~kb}$ BglII-EcoRV fragment of pQL1022 ${ }^{7}$, a pSET152 derivative previously constructed for colA2 expression under the control of PermE* (the constitutive promoter for expressing the erythromycinresistance gene in Saccharopolyspora erythraea). The resulting recombinant plasmid pQL1054, which carries the chimeric gene col/caeA2 coding for the hybrid protein that harbors the PKS module from ColA2 and the NRPS module from CaeA2, was transferred by conjugation into the $\triangle$ colA2 $S$. roseosporus mutant $\operatorname{strain}^{7}$, yielding the recombinant strain QL2006. The fermentation of QL2006 and the examination of CAE or COL production were conducted according the methods described previously ${ }^{7}$. 


\section{SUPPLIMENTARY FIGURES}

Supplementary Figure 1. Coomassie-stained SDS-PAGE analysis of purified recombinant proteins.

Lane M, protein standard. The target proteins are indicated in the dashed rectangles.

(a) Proteins produced in E. coli BL21(DE3). Lane 1, CaeA2 ${ }^{\mathrm{F} 2042 \mathrm{~L}}$ (269 kDa); Lane 2, PCP $\mathrm{CaeA}$ (derived from CaeA2, 13 kDa); Lane 3, Trx-tagged CtcaeA2 (58 kDa), Lane 4, CaeB1 (43 kDa); Lane 5, MBPfused CaeB1 (87 kDa), Lane 6, ColB1 (43 kDa); Lane 7, ColG2 (39 kDa); and Lane 8, CaeA4 (25 $\mathrm{kDa})$.

a
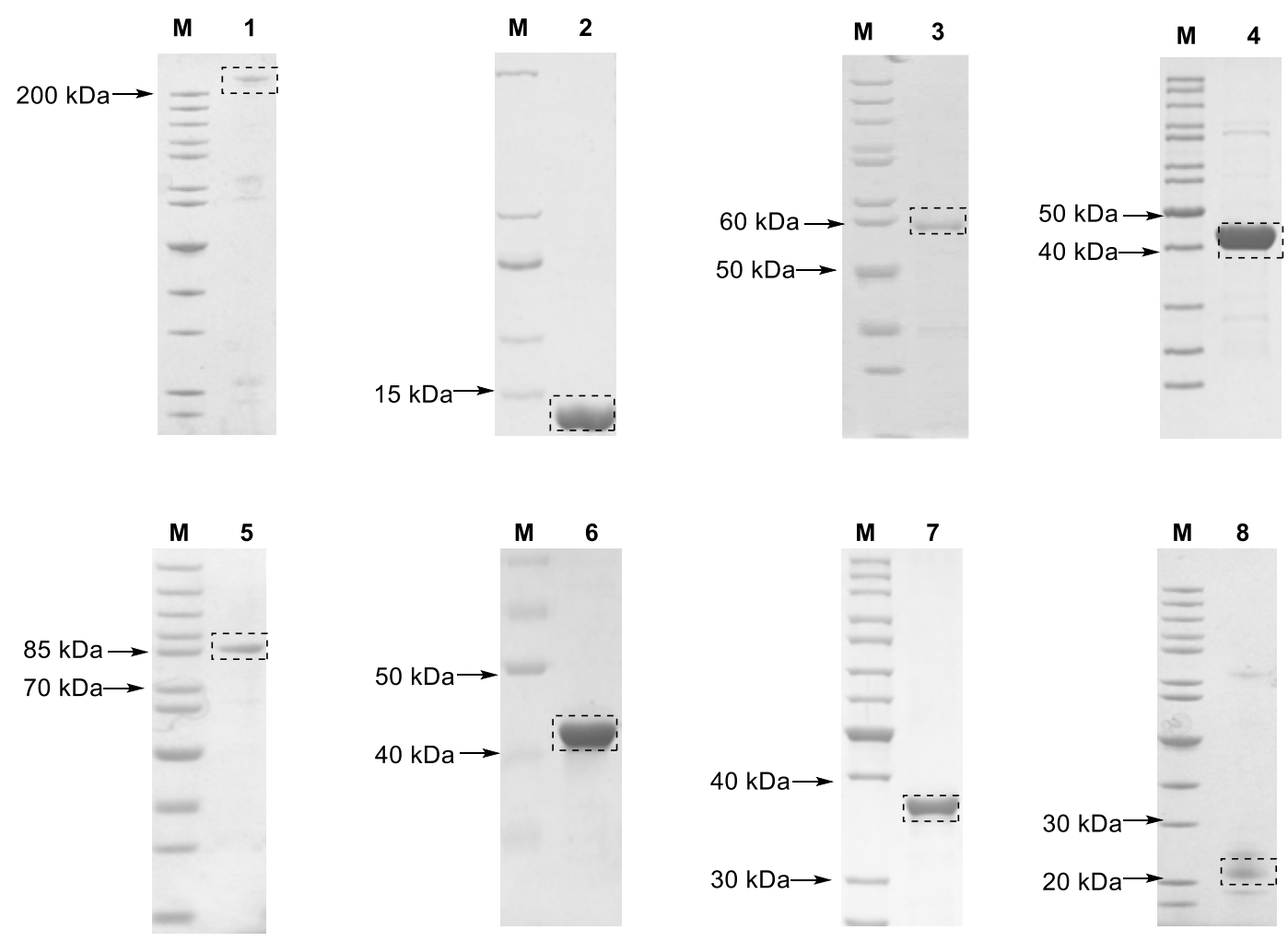
(b) Proteins produced in E. coli BAP1. Lane 1, CaeA1 (69 kDa); Lane 2, CaeA3 (118 kDa); Lane 3, ColA3 (121 kDa); Lane 4, CaeA2 (269 kDa); Lane 5, CaeA2 ${ }^{\mathrm{F} 2042 \mathrm{~L}}(269 \mathrm{kDa})$; Lane 6, CaeA2 ${ }^{\mathrm{F} 2042 \mathrm{I}}$ (269 kDa); Lane 7, CaeA2 ${ }^{\mathrm{F} 2042 \mathrm{~V}}(269 \mathrm{kDa})$; Lane 8, CaeA2- $\triangle \mathrm{Ct}(225 \mathrm{kDa})$; Lane 9, CaeA2 ${ }^{\mathrm{F} 2042 \mathrm{~L}} \triangle \mathrm{Ct}$ (225 kDa); and Lane 10, CaeA3CColA3 3 -PCP $(120 \mathrm{kDa})$.

b
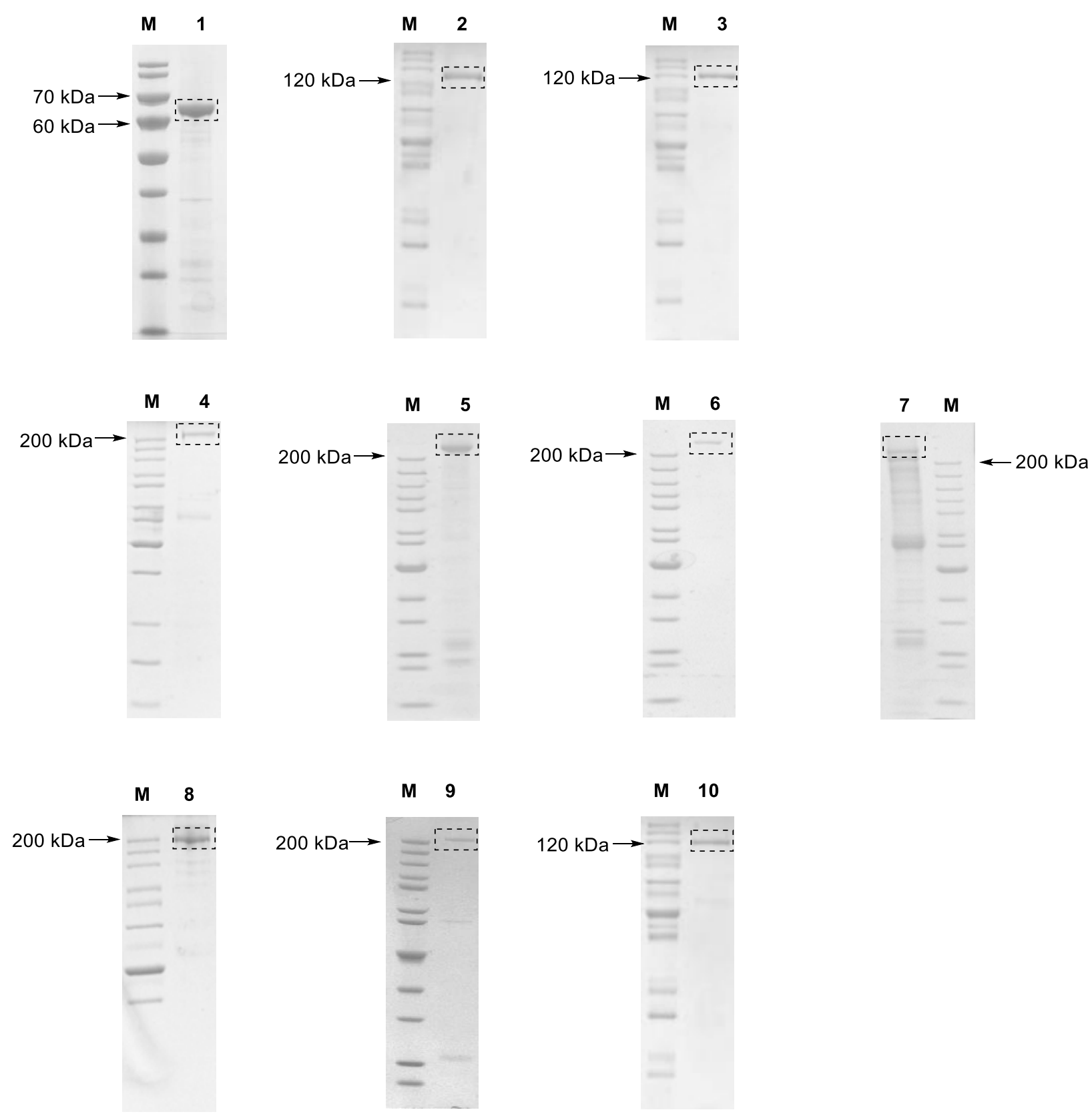
Supplementary Figure 2. Supplementary data for in vitro reconstitution of the 2,2'-bipyridine assembly line.

(a) Determination of component necessity in the production of the CAE 2,2'-bipyridine intermediate

1. Tested reactions were derived from the combination of CaeA1, CaeA2, CaeA3 and CaeB1 with picolinic acid, malonyl-S-CoA, L-cysteine, L-leucine, and ATP (i), and included those lacking the enzyme CaeA1 (ii), CaeA2 (iii), CaeA3 (iv) or CaeB1 (v) and the substrate picolinic acid (vi), malonylS-CoA (vii), L-cysteine (viii), L-leucine (ix) or ATP (x, for the activity of the A domains), respectively. Synthesized 1 was used as the stabdard (xi).

a
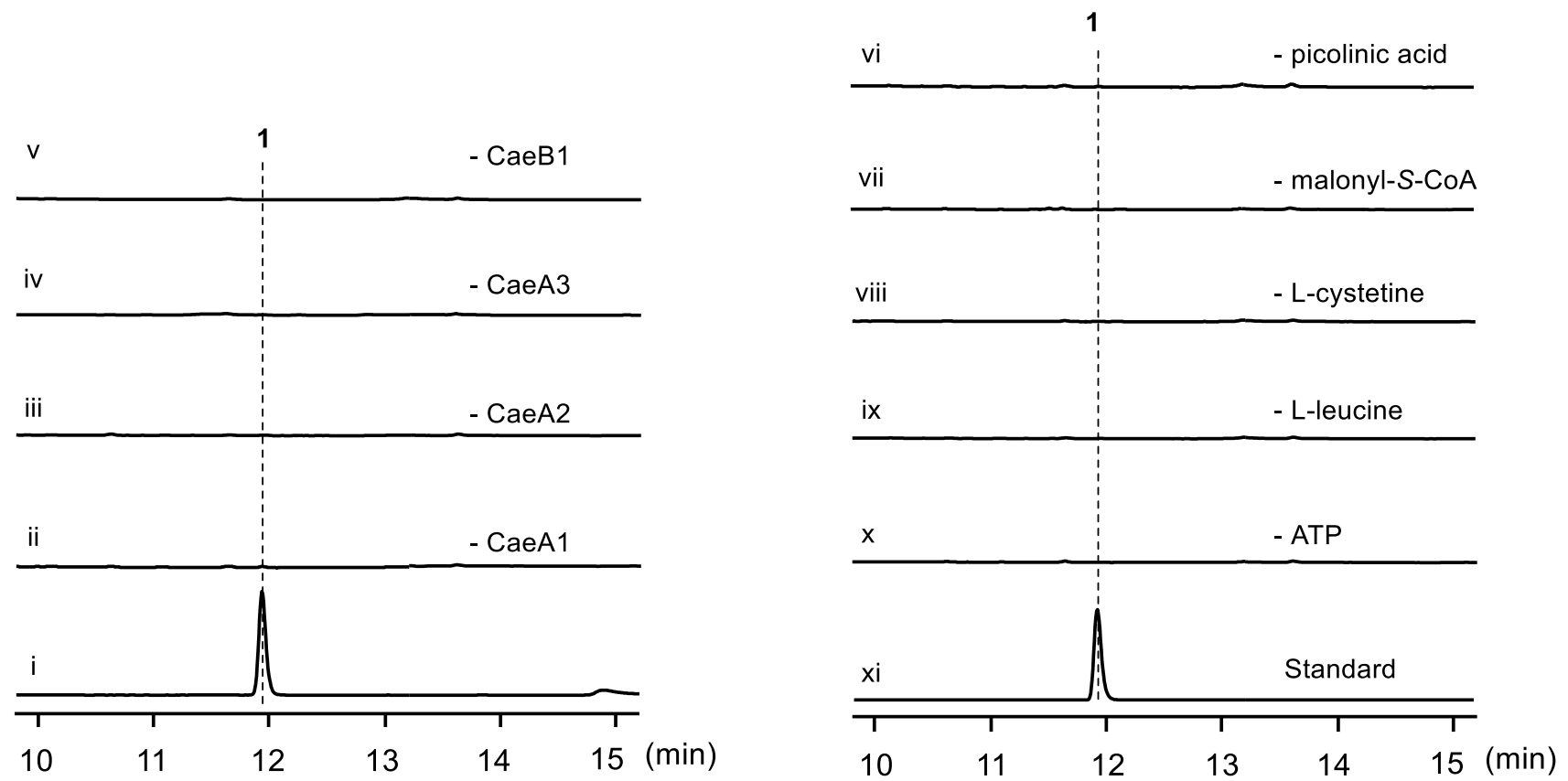
(b) Activity assays of CaeA2 variants in the production of 1 . The CaeA2 variants, i.e., CaeA2 ${ }^{\mathrm{F} 2042 \mathrm{~L}}$, $\mathrm{CaeA} 2^{\mathrm{F} 2042 \mathrm{~V}}$ and $\mathrm{CaeA} 2^{\mathrm{F} 2042 \mathrm{I}}$, were designed according to residue conservative analysis (left), which was conducted by comparing the 15-aa sequence SLGGDSIMGIQF 2042 $_{2}$ VSR that is derived from the PCP domain of CaeA2 (the Ppant-modified active-site L-serine residue and the target L-phenylalanine residue are highlighted in color and underlined) with the corresponding sequences of 500 homologous PCP domains or proteins from NCBI NR database. Tested reactions were derived from the combination of CaeA1, CaeA2, CaeA3 and CaeB1 with picolinic acid, malonyl-S-CoA, L-cysteine, L-leucine, and ATP (i), and included those in which CaeA2 was replaced with CaeA2 ${ }^{\mathrm{F} 2042 \mathrm{~L}}$ (ii), CaeA2 ${ }^{\mathrm{F} 2042 \mathrm{~V}}$ (iii) and CaeA2 ${ }^{\text {F2042I }}$ (iv), respectively.

b
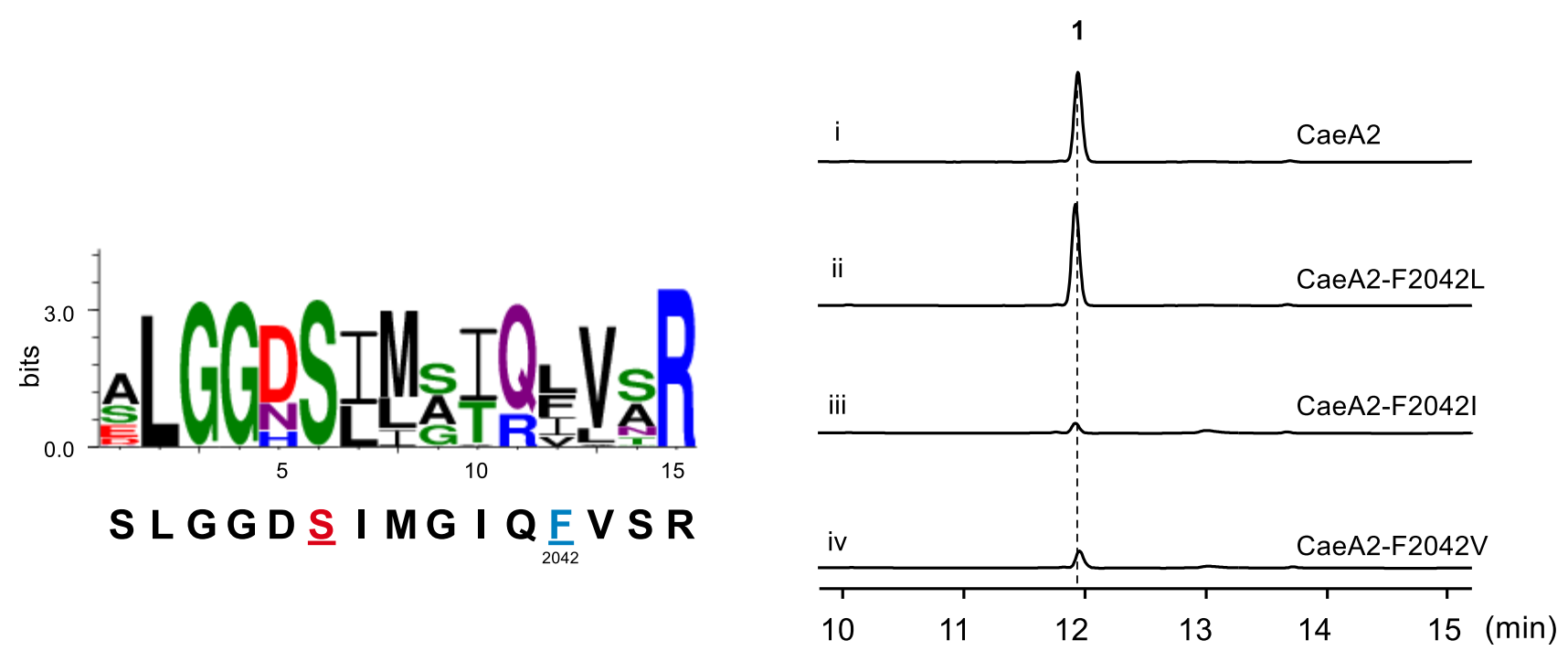
Supplementary Figure 3. Analysis of the flavoproteins CaeB1 and ColB1.

(a) UV spectra of the recombinant proteins CaeB1 and ColB1 that were purified from E. coli BL21(DE3).

(b) Determination of flavin cofactors associated with CaeB1 and ColB1. (i) authentic FMN; (ii) authentic FAD, (iii) boiled CaeB1, (iv) boiled CaeB1-S168A, (v) boiled CaeB1-E372A, and (vi) boiled ColB1. For examination by HPLC, UV absorbance at $375 \mathrm{~nm}$.

(c) Activity assays of $\mathrm{CaeB} 1$ variants in the production of $\mathbf{1}$. Tested reactions were derived from the combination of CaeA1, CaeA2, CaeA3 and CaeB1 with picolinic acid, malonyl-S-CoA, L-cysteine, Lleucine, and ATP (i), and included those in which CaeB1 was replaced with CaeB1-E372A(ii), CaeB1S168A (iii), and CaeB1-S168A with excess of FAD (iv), respectively.

(d) Sequence alignment of $\mathrm{CaeB} 1$ and ColB1 with various FAD-dependent dehydrogenases. The homologs include FkbI (1R2G) in FK520 biosynthesis $^{8}$, medium chain acyl-CoA dehydrogenase $(\mathrm{MCAD}, \text { 1T9G_A })^{9}$, short chain acyl-CoA dehydrogenase (SCAD, 1JQI_A) ${ }^{10}$ and cyclohexanecarboxyl-CoA dehydrogenase (ChCAD, ABC76100.1) ${ }^{11}$. Residues important for dehydrogenase activity, FAD-binding and phosphopentatheine binding are indicated by star, black triangle and red triangle, respectively. 
a
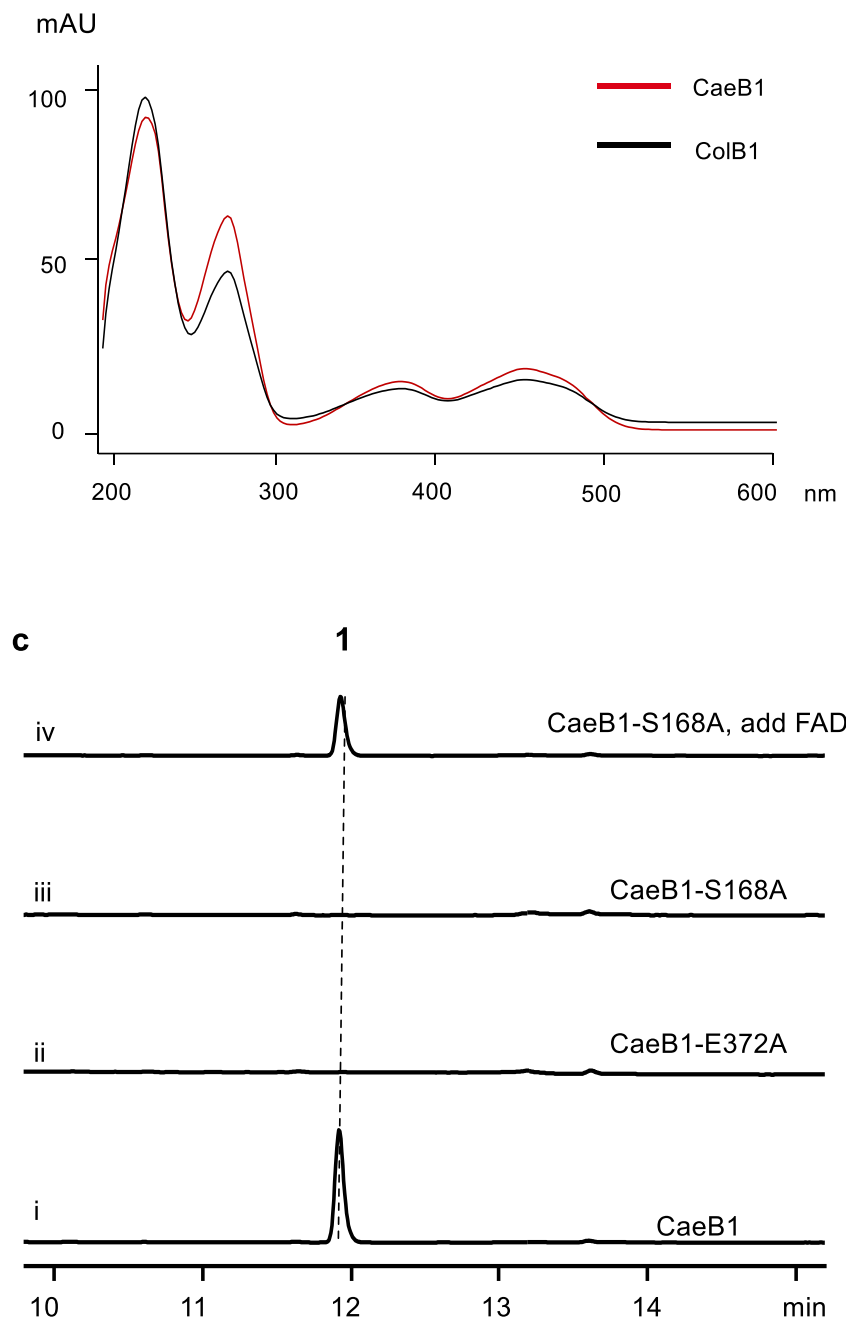

b
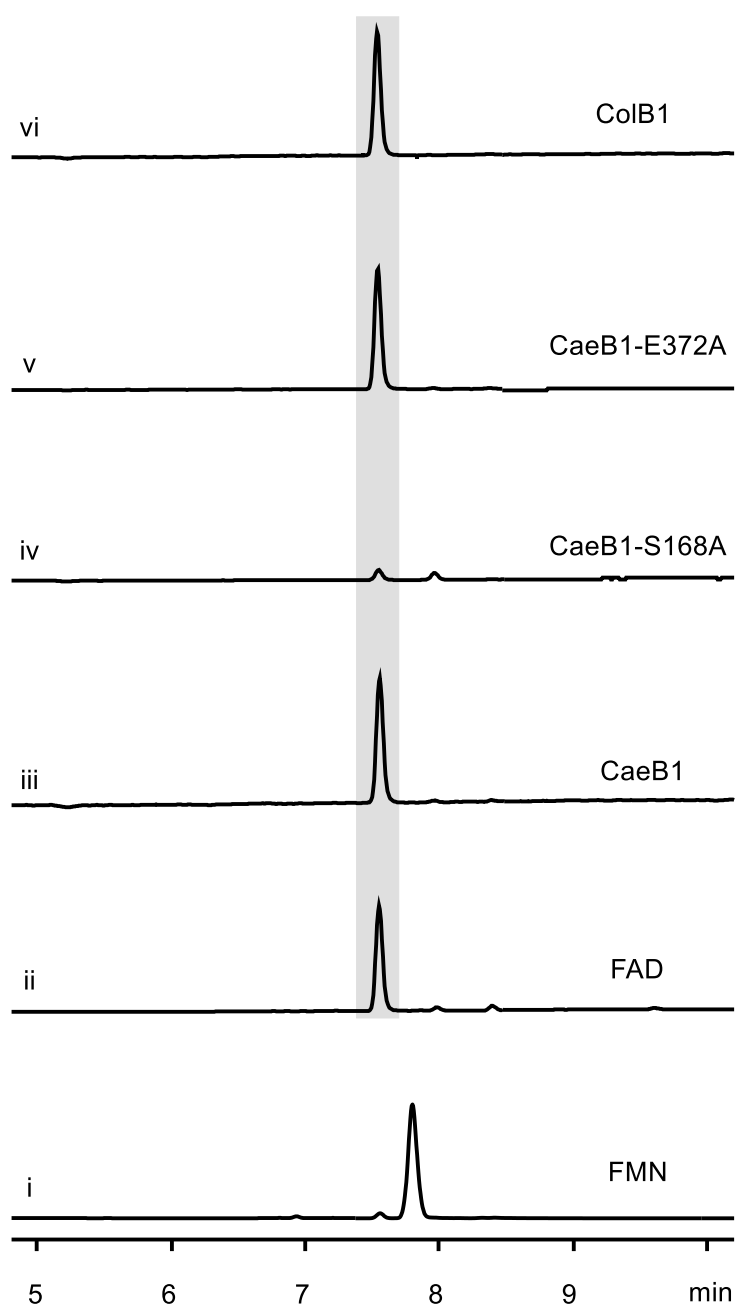
bioRxiv preprint doi: https://doi.org/10.1101/2020.12.07.415471; this version posted December 8, 2020. The copyright holder for this

preprint (which was not certified by peer review) is the author/funder. All rights reserved. No reuse allowed without permission.

d

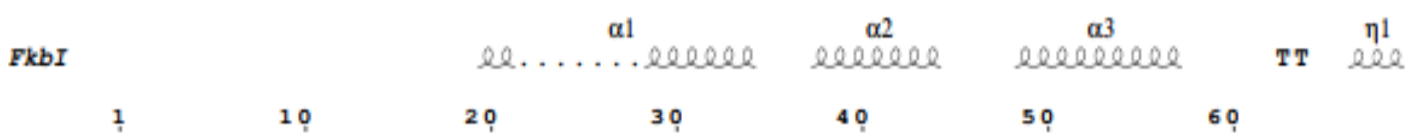

CaeB1 M.TTGNGAAPPLLTRLSEDEAWRAHVRAFÄEH APLSDRMDRDATLDPLRERLFSAGLMAVAPARY

ColB1 NTNPGQSATDATDHPDSENQRWRERIRHFAEKEIAPTSTTMDRTATTDAGTRERTFAEGTSVEIPRGY

FKbI M................. TERDAGDRAAEWDTSGELPRDLLVRLGADGLLCAEVAAEH

MCAD KANRQREPGLGFSEEFTEQOKEFOATARKFAREEI I PVAAEYDKTGEYPVPLIRRAWELGLMNTHIPENC

SCAD L....... HSVYQSVELPETHOMLRQTCRDFAEKELVP I AAQLDKEHLFP T SVKKMGELGLLAMDVPEEL

ChCAD M...........YINTETEDLKTAIDAIRKAVRRIAPLAAEVDSGVIKPEIYDLLWDLGLMUTYPEYY

$F k b I$

$\alpha 4$

80

90

25

70

100

sereeceeve

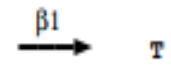

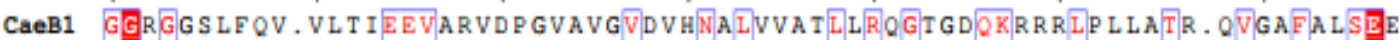

ColB1 GGTGGTCQL. ITTIEEVARVDPGVAVGVHVHNVTVAGTTTRHASGQRRQYTPQTATG.KIGAFATSEE FkbI GGLGLGSREN.GEFTAHVGSLCSSLRSVMTS. OGMAAWTVRLGDAGQRATFLKELTSG. KLAAVGFSER MCAD GGLGLTFDA.CLISEELAYGCTGVQTAIEG. NSIGQMPIIIAGNDQQKKKYLGRMTEEPLMCAYCVTEP SCAD SGAGLDYLAY.SIALEEISRGCASTGVIMSVNNSLYLGPILIKGSSQOKOQWITPETNGDKIGCFALSEP ChCAD GGSETNPGTLLCIGCEEIAKACASTALLII. QAVGSF LMHGGRELDR I A R I VNNRELAYLVSE

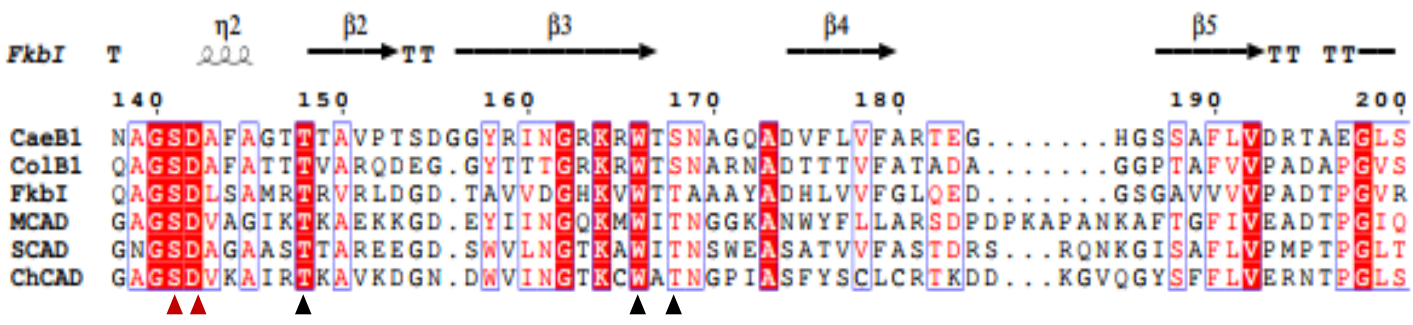

B6

FkbI

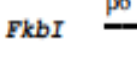

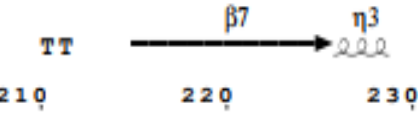

$\eta^{3}$ 230
TTT

240

$\alpha 7$

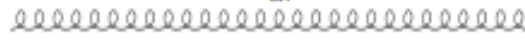

CaeB1 IDPRTEOMTVRAAATADVVILTDVRVRRODCVGGPGGGEGVAIGA. LDIGRIGIAAOMVGLĀOGALDEAVA

ColB1 TDDRVEQMGVRAAAT SDVIFDGTPVRTAQRVGPPGGGQTVASG.TGTGRTGIAAQMTGTAQGATDAATG FKbI VERVPKPSGCRAAGHADLHLDQVRVPAGAVLAGSGASLPMLVAASLAYGRKSVAWGCVGILRACRTAAVA MCAD IGRKELNMGORCSDIRGIVFEDVKVPKENVLIGDGAGFKVAMGA.FDKTRPVVAAGAGLAQRALDEATK

SCAD LGKKEDKLGIRASSTANLIFEDCR IPKENLLGEPGMGEKI AMQT. LDMGRIGIASQALGI AQASLDCAVK

ChCAD VGKIEHKMGMRGSOISEVILEDVRVPAENLLGELNMGKLAMKD. FDMSRPAIAAQALGISEGAFAQMET $\Delta \boldsymbol{\Delta}$

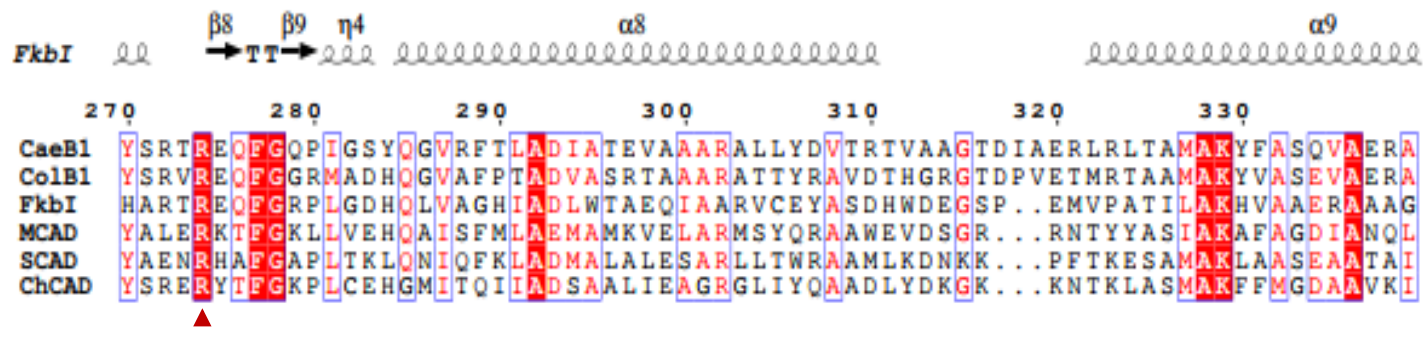

$\eta 5$

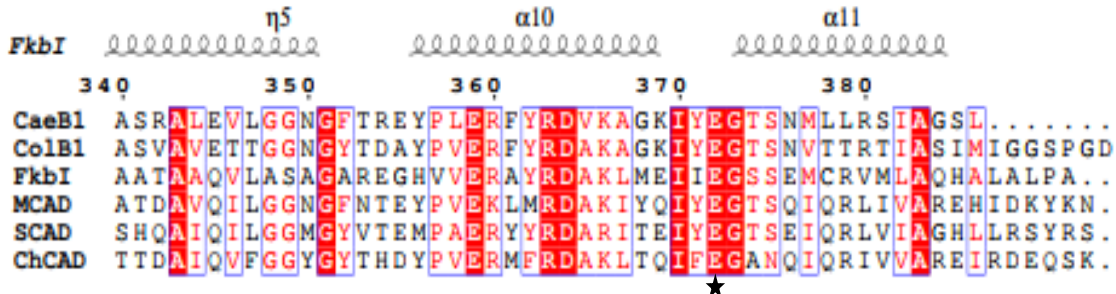


Supplementary Figure 4. Determination of the 15-aa sequence that is derived from the PCP domain of CaeA2 and contains the Ppant-modified active-site L-serine residue (red) by nanoLC-MS/MS. (a) Ppant-modified CaeA2 by treatment with trypsin (complete digestion) and chymotrypsin (partial digestion). (b) Ppant-modified CaeA2 $2^{\mathrm{F} 2042 \mathrm{~L}}$ by treatment with trypsin (complete digestion) and chymotrypsin (complete digestion).

a

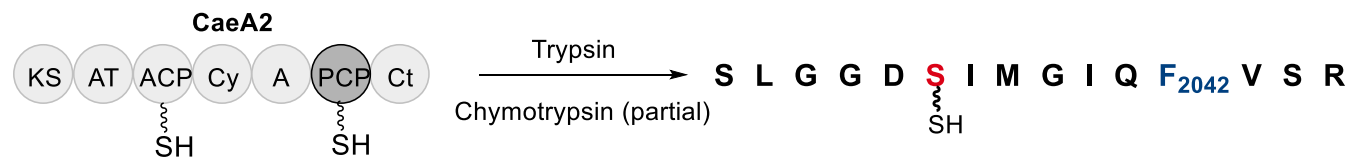

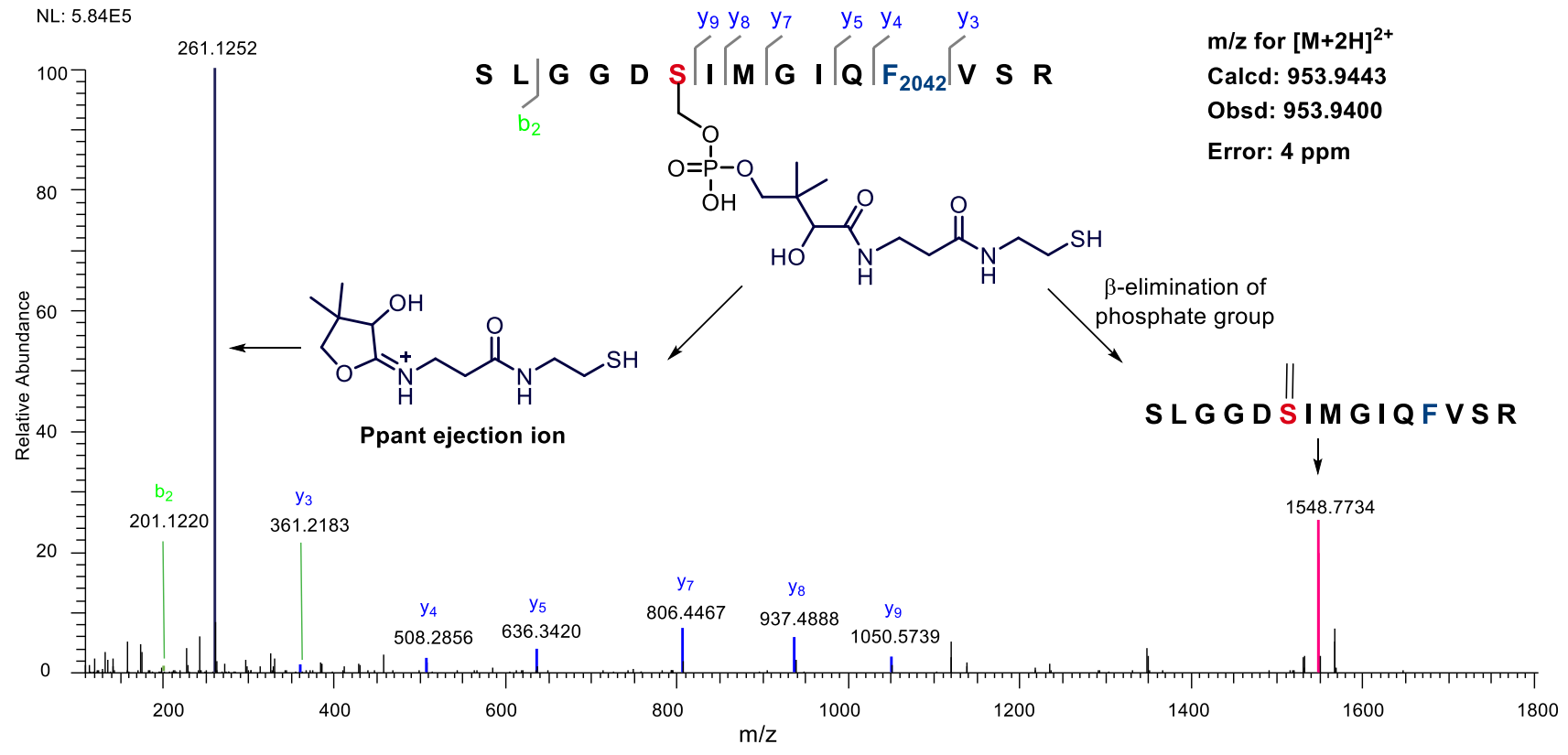


b

CaeA2 ${ }^{\mathrm{F} 2042 \mathrm{~L}}$

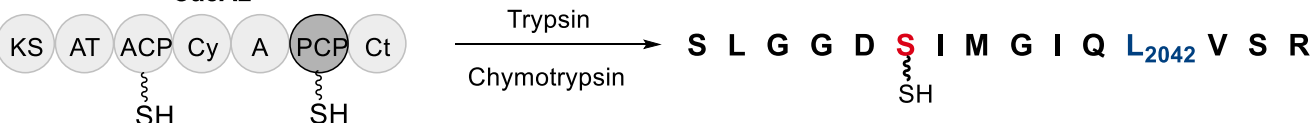

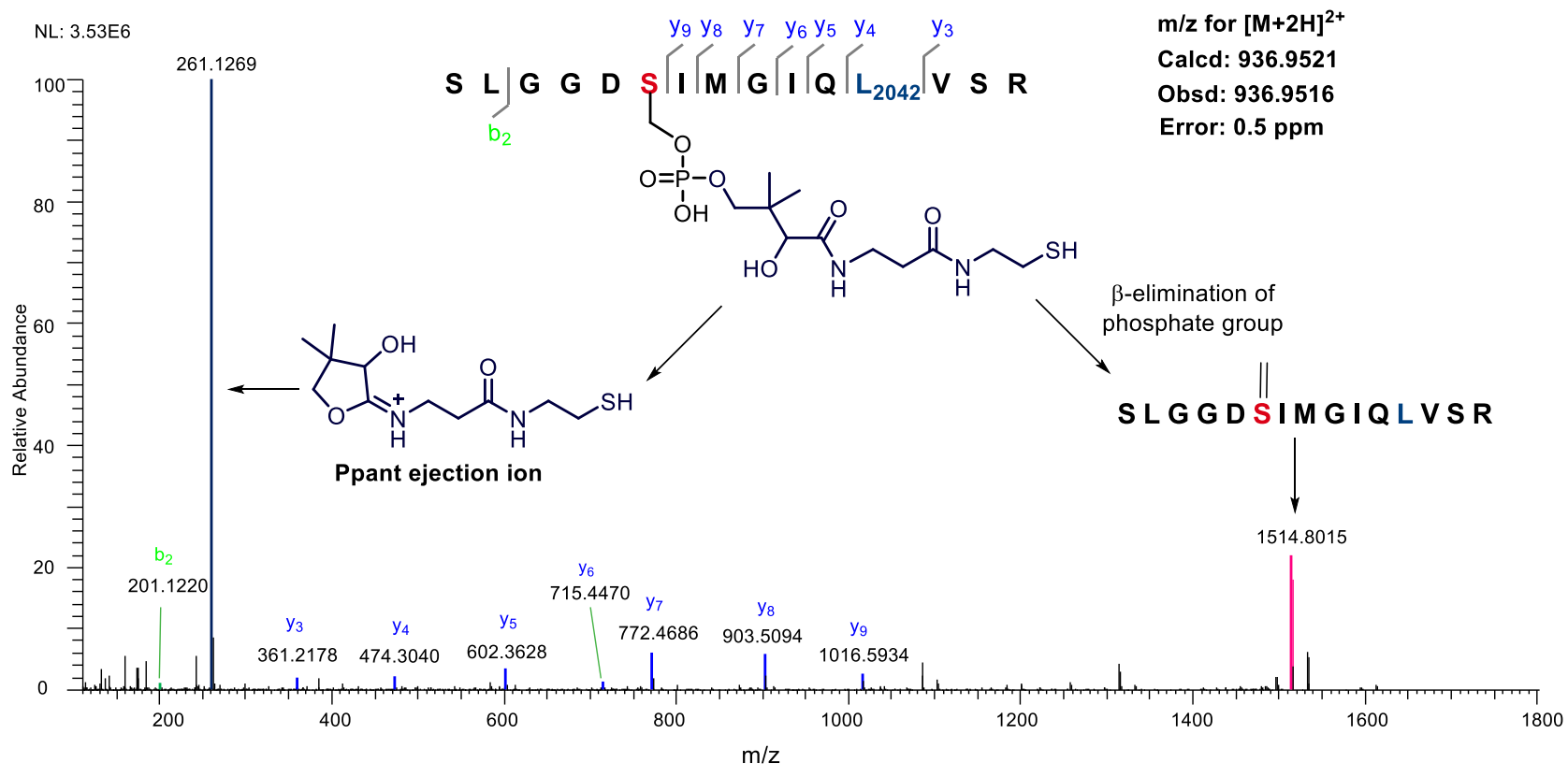


Supplementary Figure 5. Characterization of L-cysteinyl-S-CaeA2 $2^{\mathrm{F} 2042 \mathrm{~L}}$ (3) by nanoLC-MS/MS following complete digestion with trypsin and chymotrypsin. (a) Incubation of thiolated CaeA2 $2^{\mathrm{F} 2042 \mathrm{~L}}$ and L-cysteine. (b) Treatment of with IAM after the incubation of thiolated CaeA2 $2^{\mathrm{F} 2042 \mathrm{~L}}$ and L-cysteine.

a

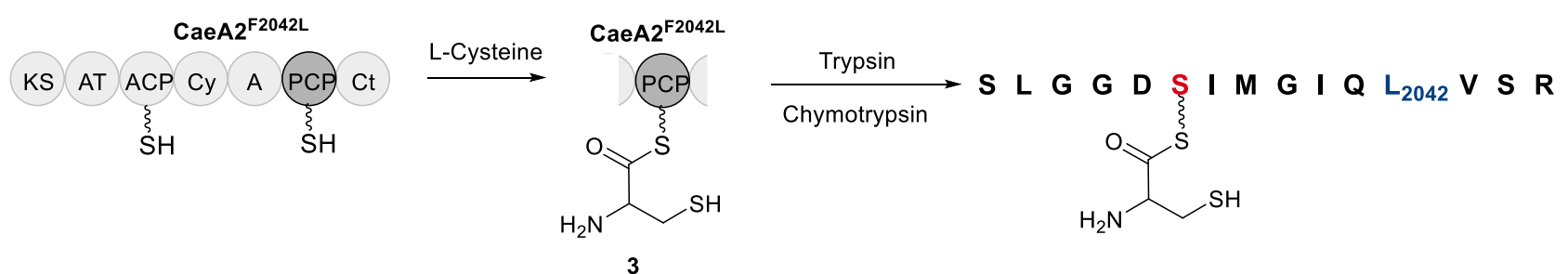

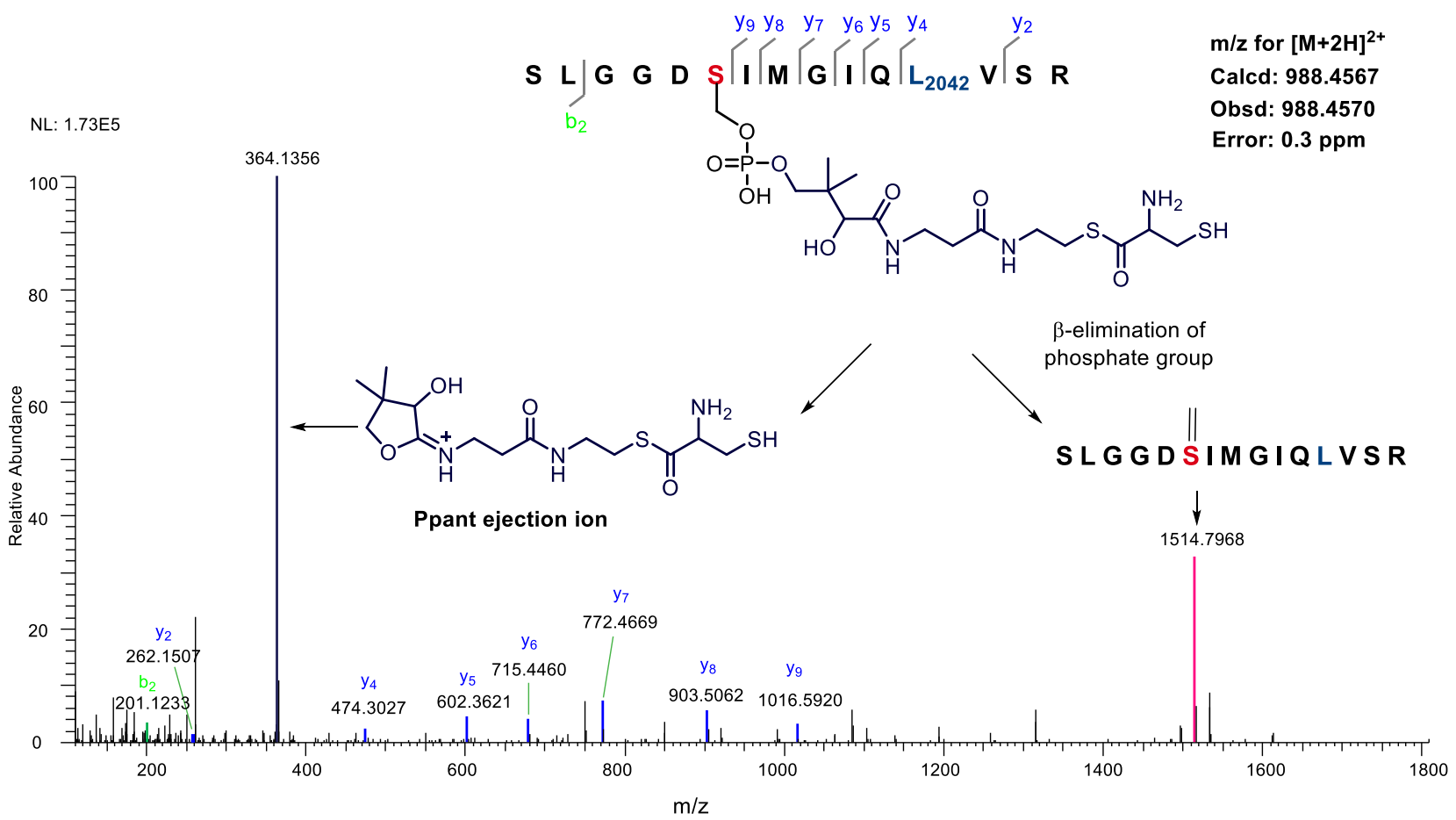


b

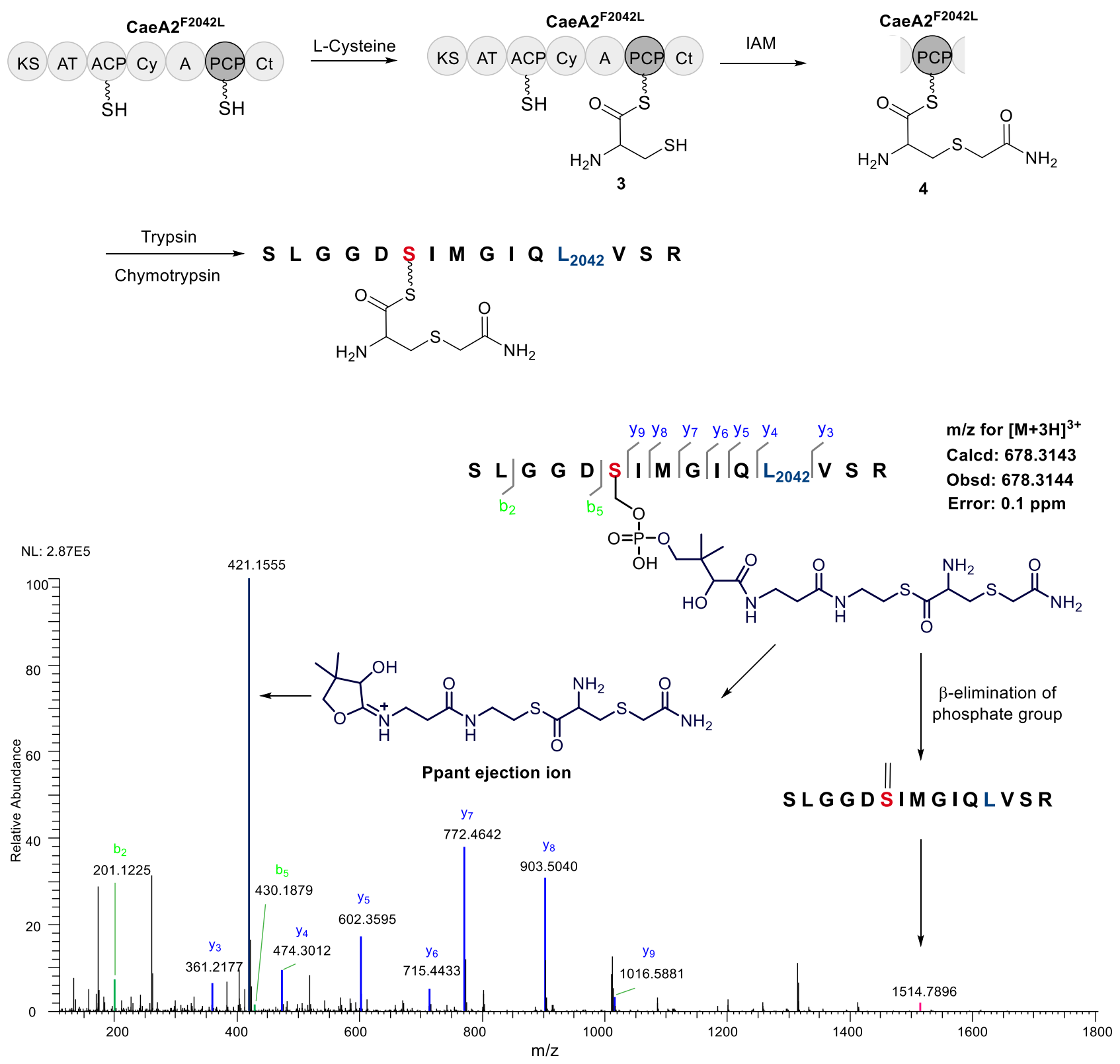


Supplementary Figure 6. Characterization of (3-sulfhydryl)-pyruvoyl-S-CaeA2 ${ }^{\mathrm{F} 2042 \mathrm{~L}}$ (6) by nanoLCMS/MS following treatment with IAM and subsequent complete digestion with trypsin and chymotrypsin.

(a) Incubation of thiolated CaeA2 ${ }^{\mathrm{F} 2042 \mathrm{~L}}, \mathrm{CaeB} 1$ and L-cysteine. MS-detectable products include 4 (i) and 5 (ii).

a
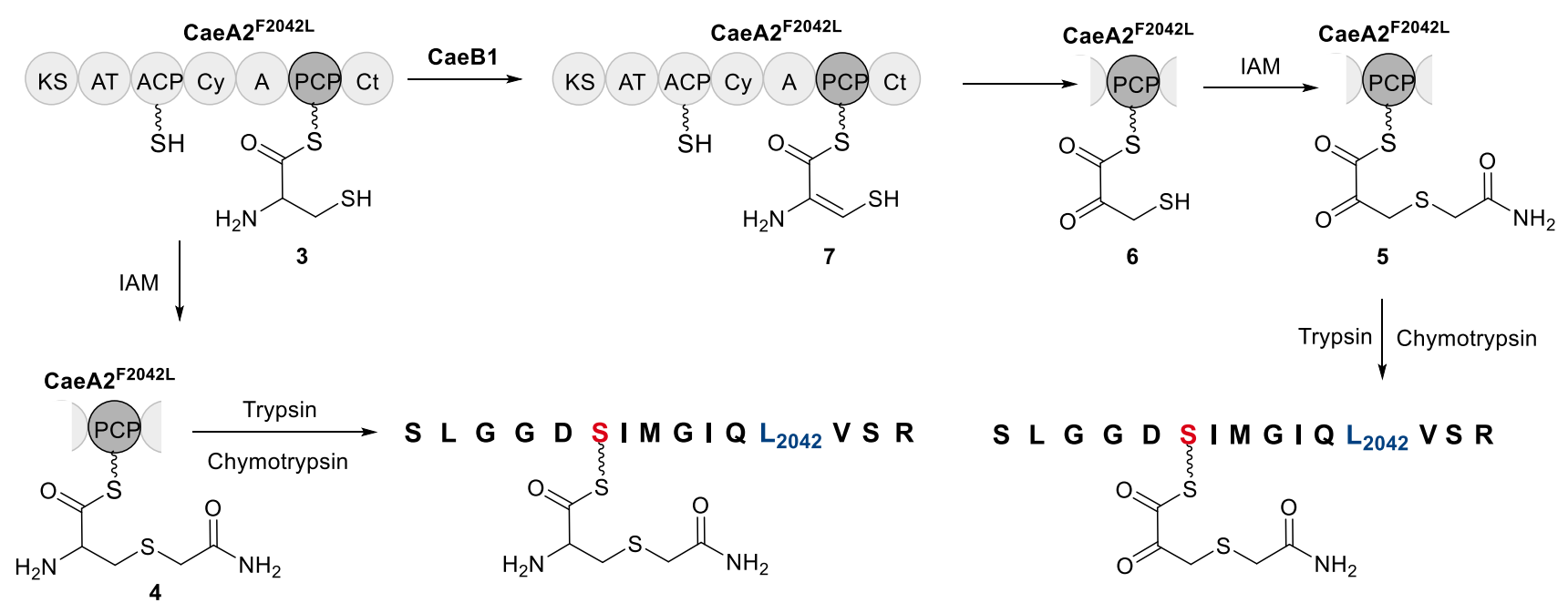

i

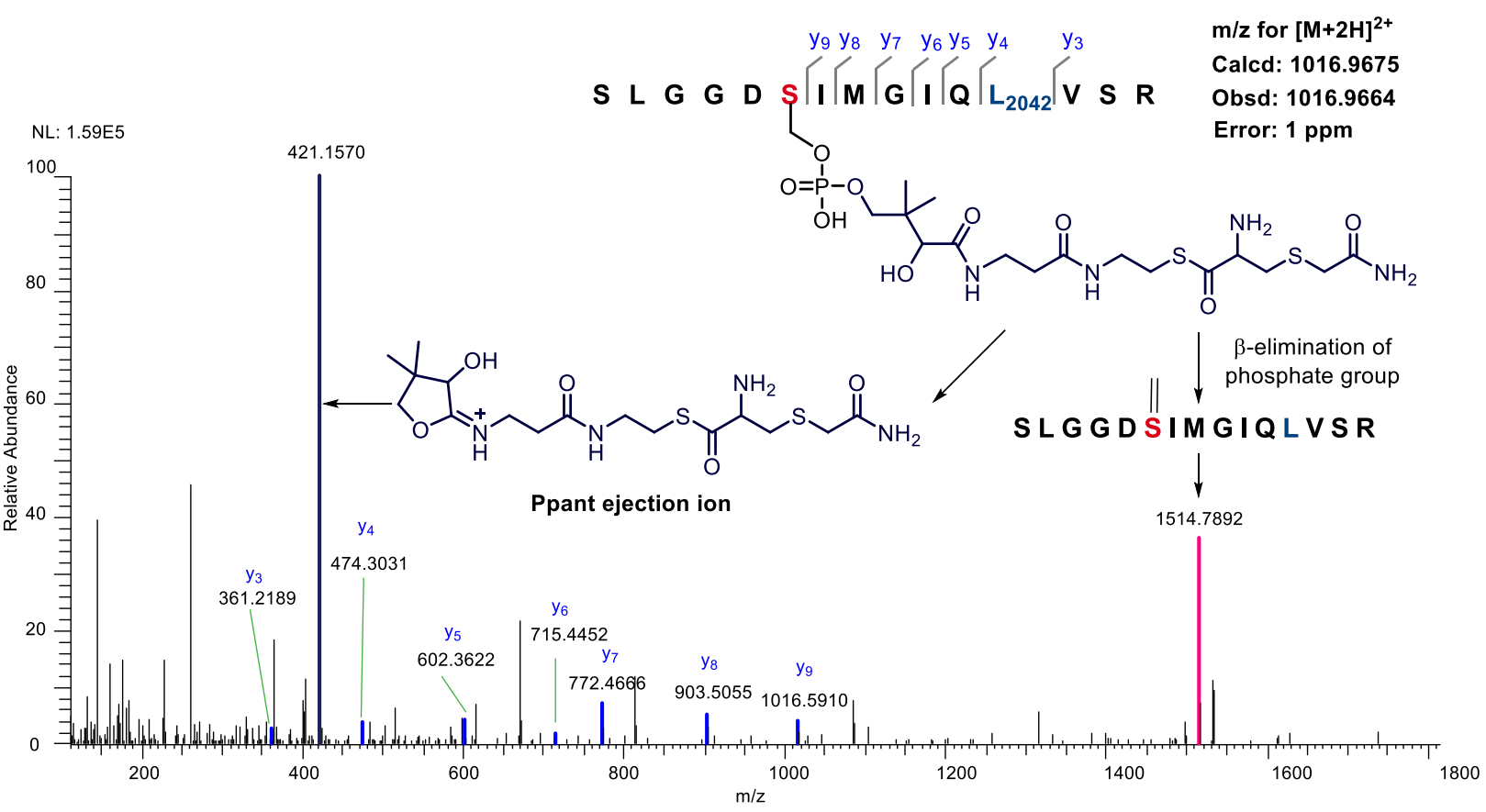


ii

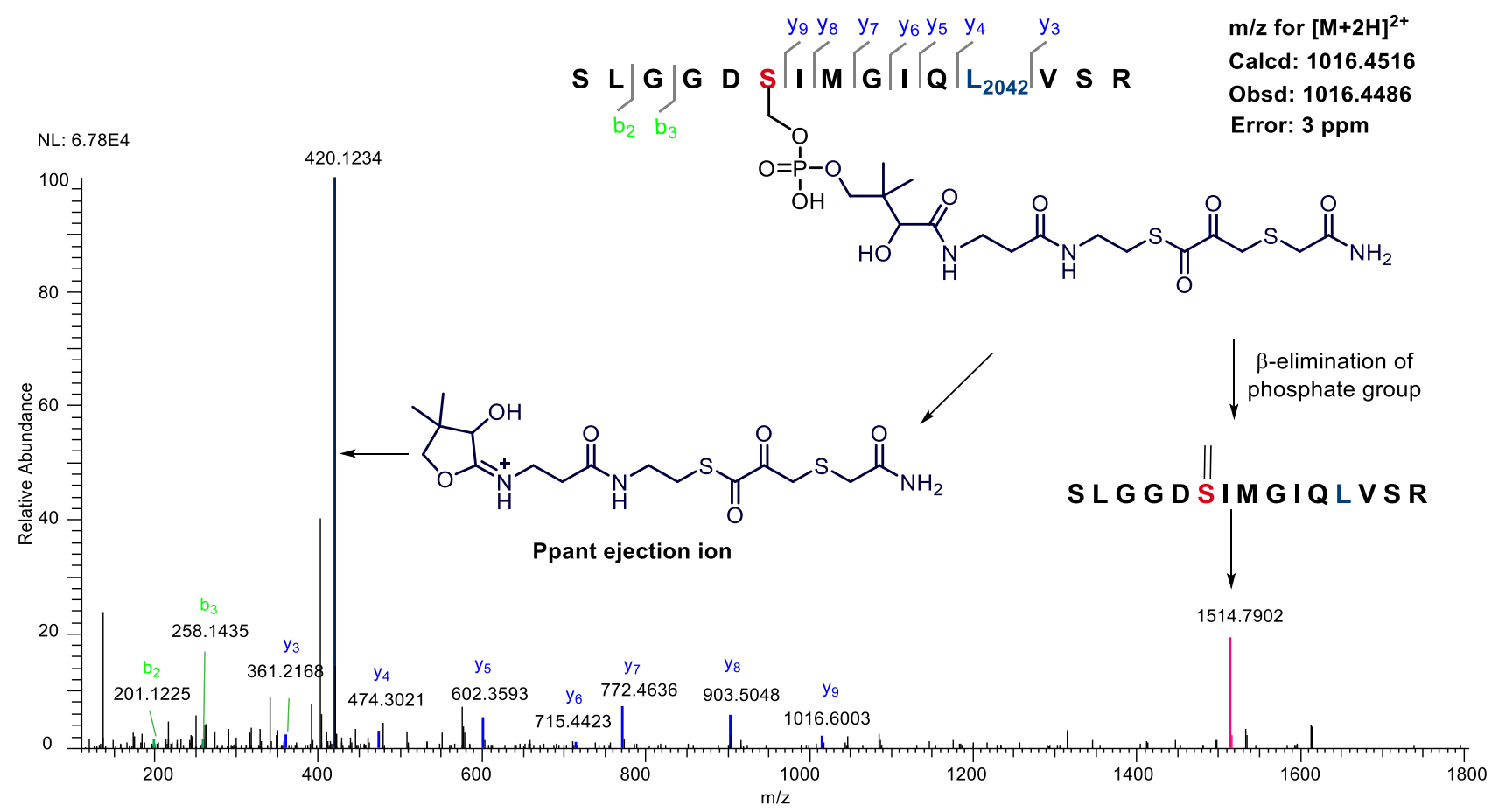

(b) Incubation of thiolated $\mathrm{CaeA} 2{ }^{\mathrm{F} 2042 \mathrm{~L}}$, CaeB1 and $\mathrm{L}-\left[1,2,3-{ }^{13} \mathrm{C}_{3},{ }^{15} \mathrm{~N}\right]$ cysteine. MS-detectable products include ${ }^{15} \mathrm{~N}$ and/or ${ }^{13} \mathrm{C}$ labelled 4 (i) and 5 (ii).

b

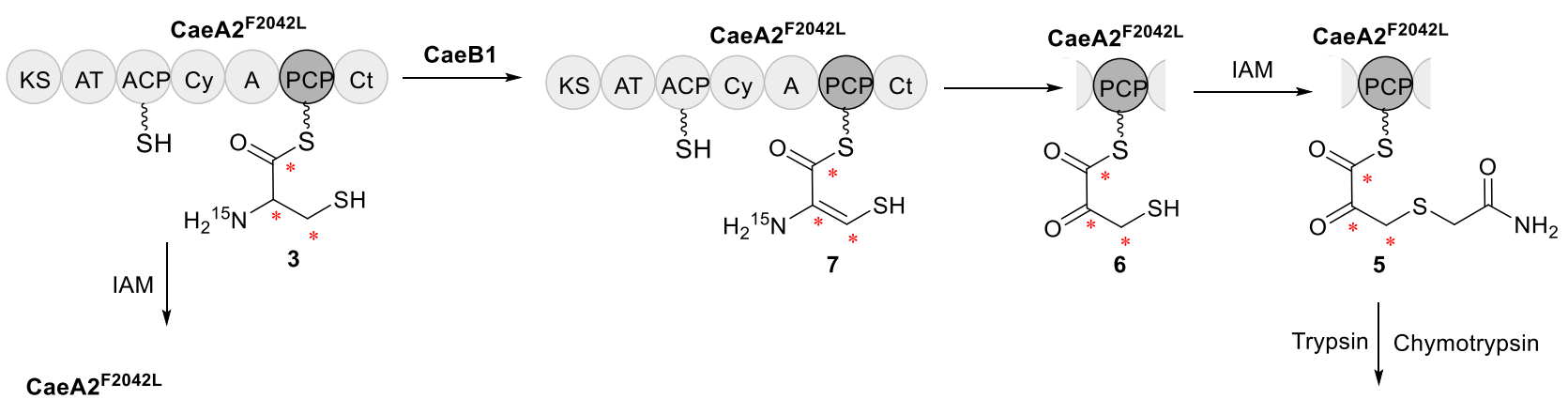

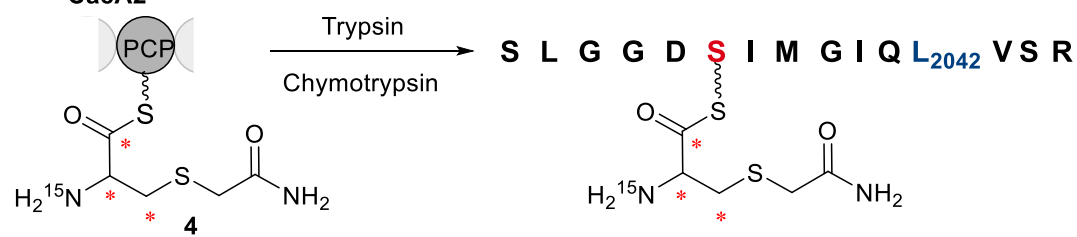

S L G G D S I M G I Q L L 2042 VS R<smiles>CSC(=O)C(=O)CSCC(N)=O</smiles> 
i

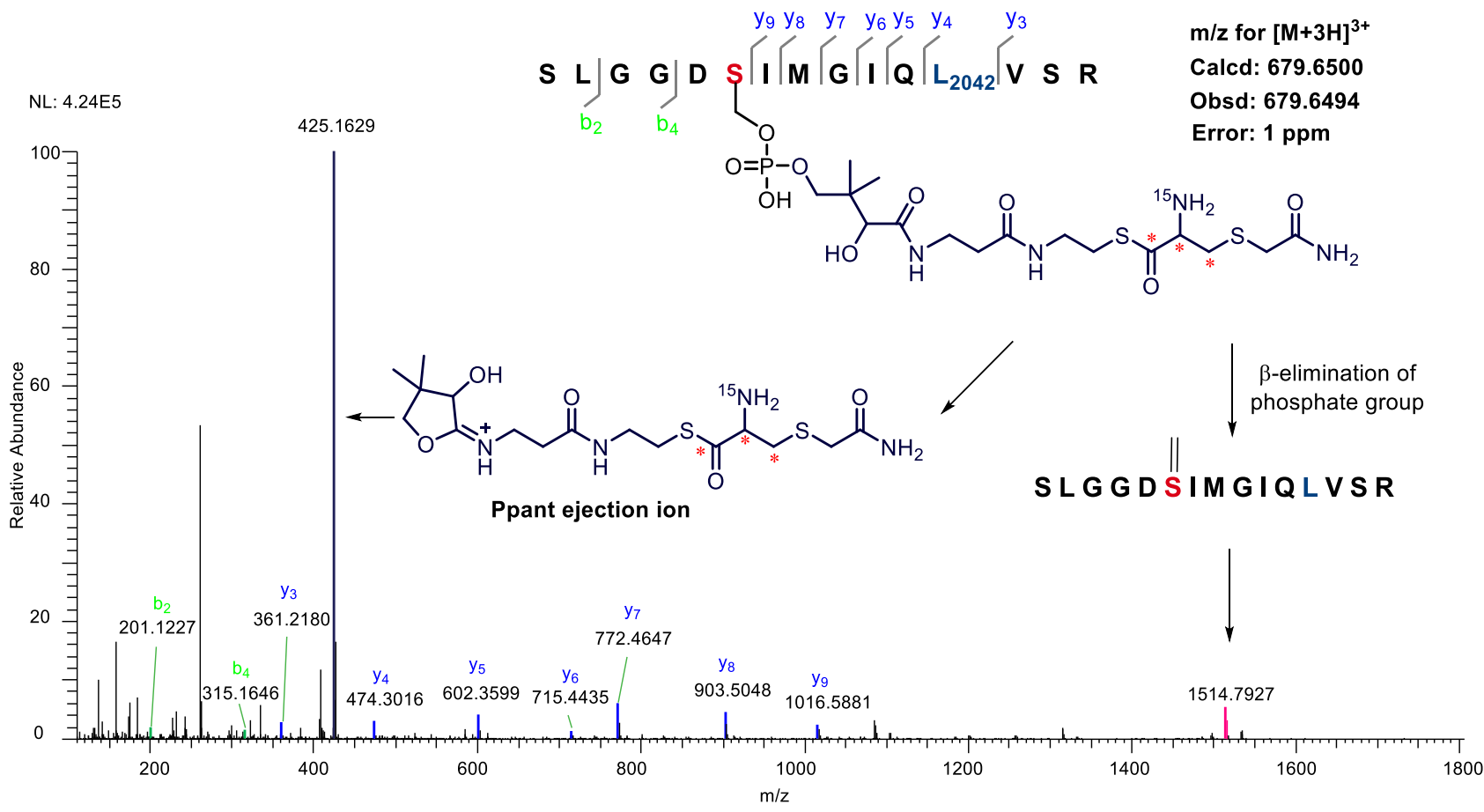

ii

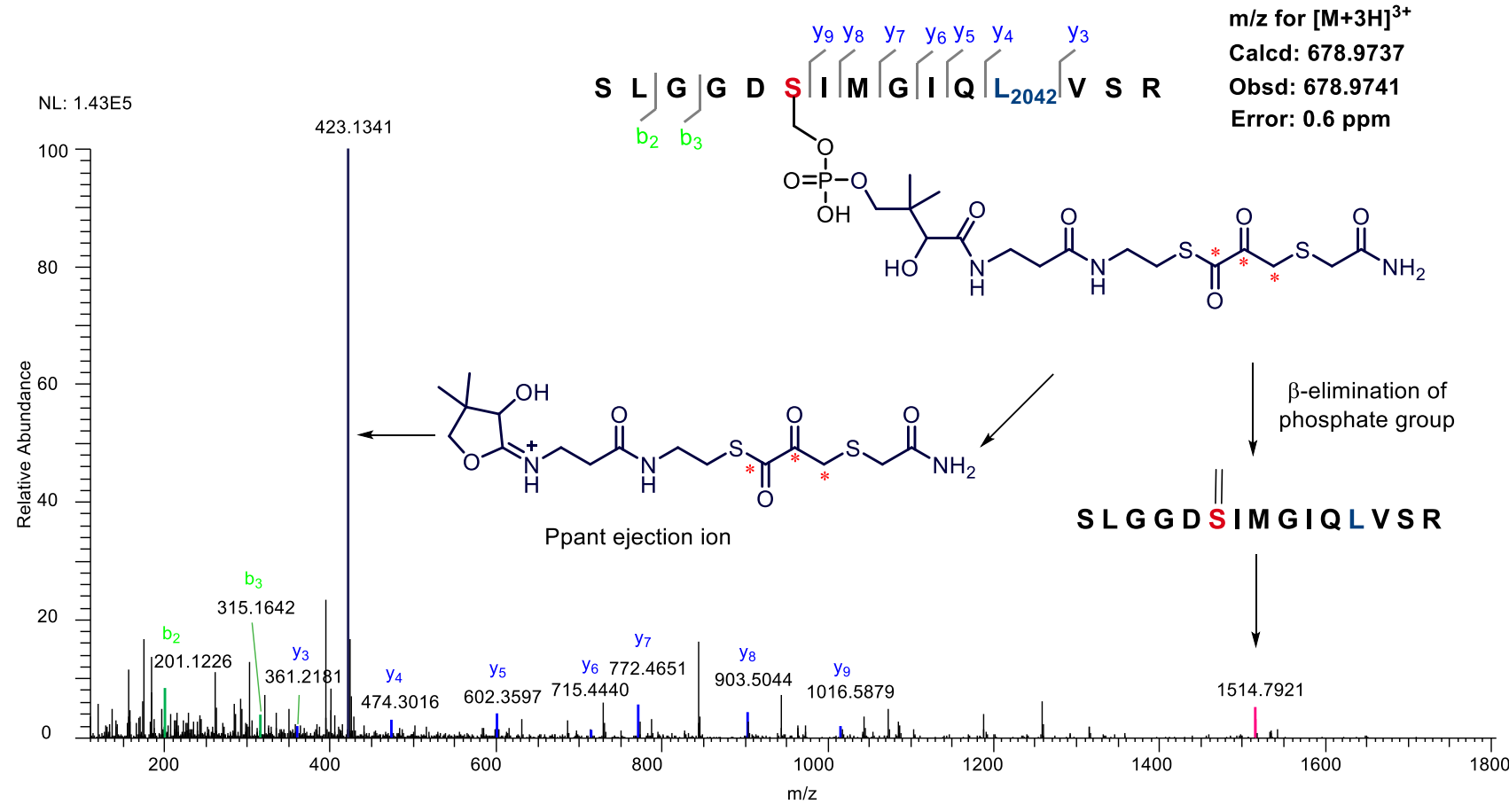

(c) Incubation of thiolated $\mathrm{CaeA} 2^{\mathrm{F} 2042 \mathrm{~L}}, \mathrm{CaeB} 1$ and $\mathrm{L}-\left[2,3,3-\mathrm{D}_{3}\right]$ cysteine. MS-detectable products include labelled 4 (i) and unlabeled 5 (ii). 
C

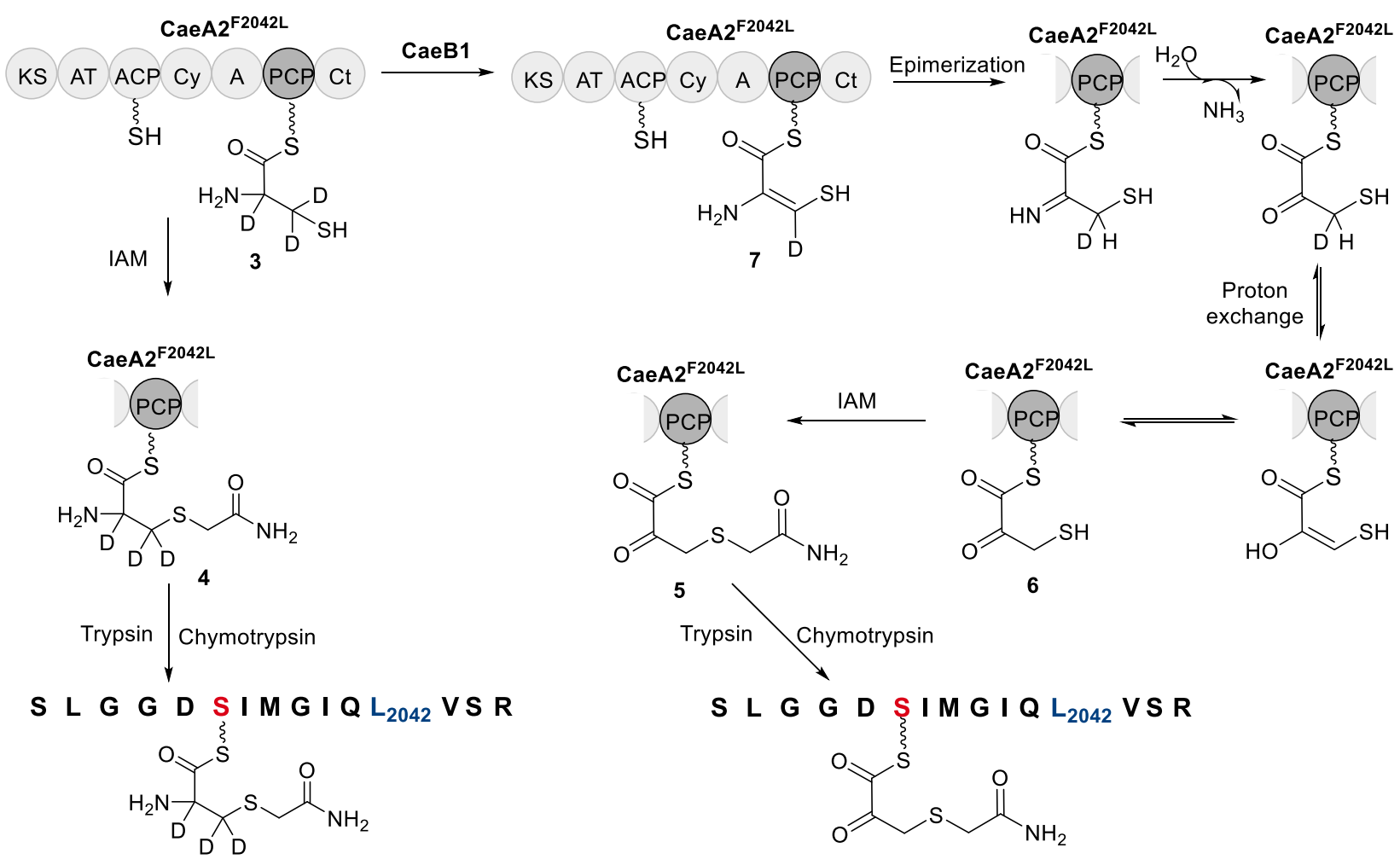

i
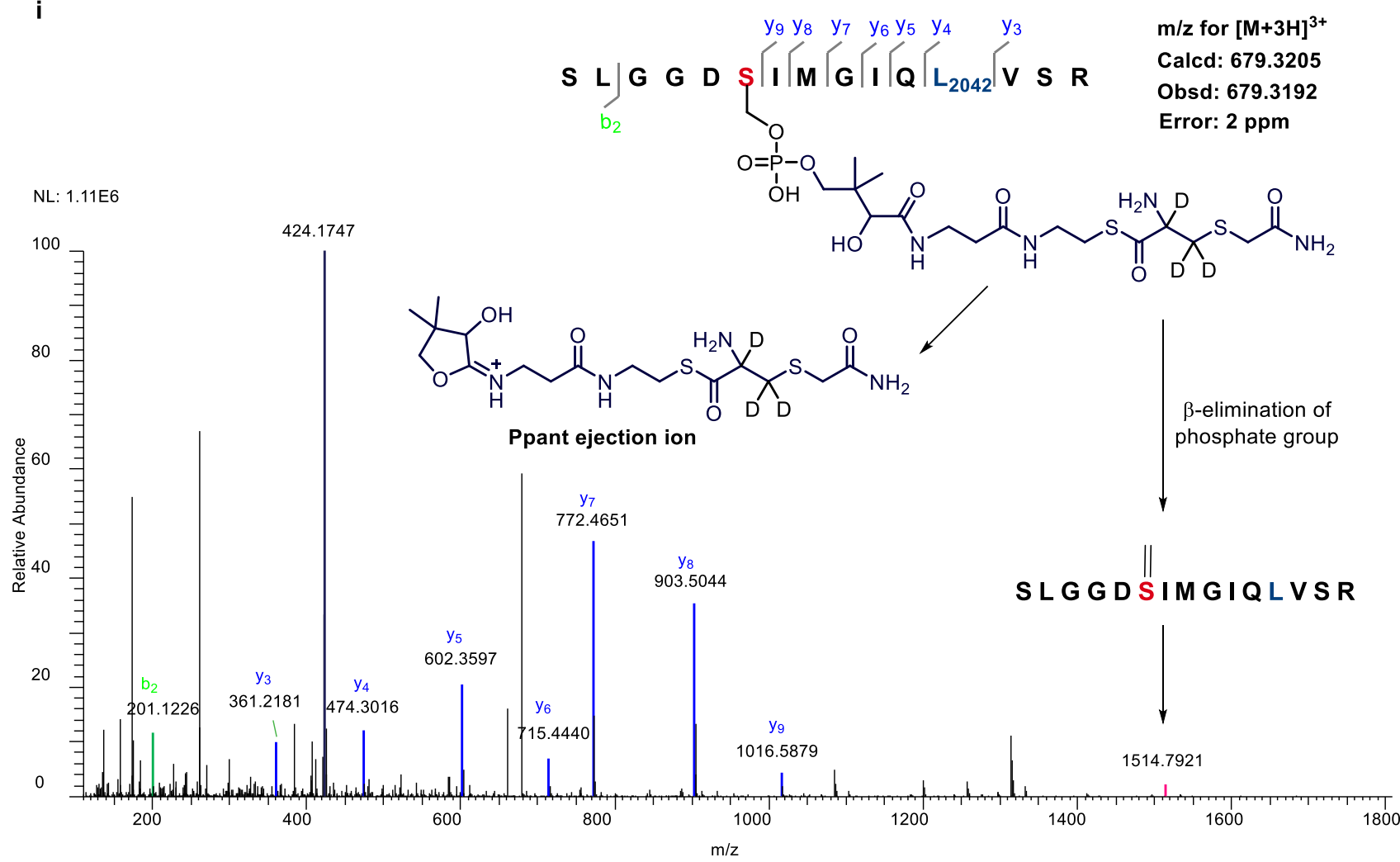
ii

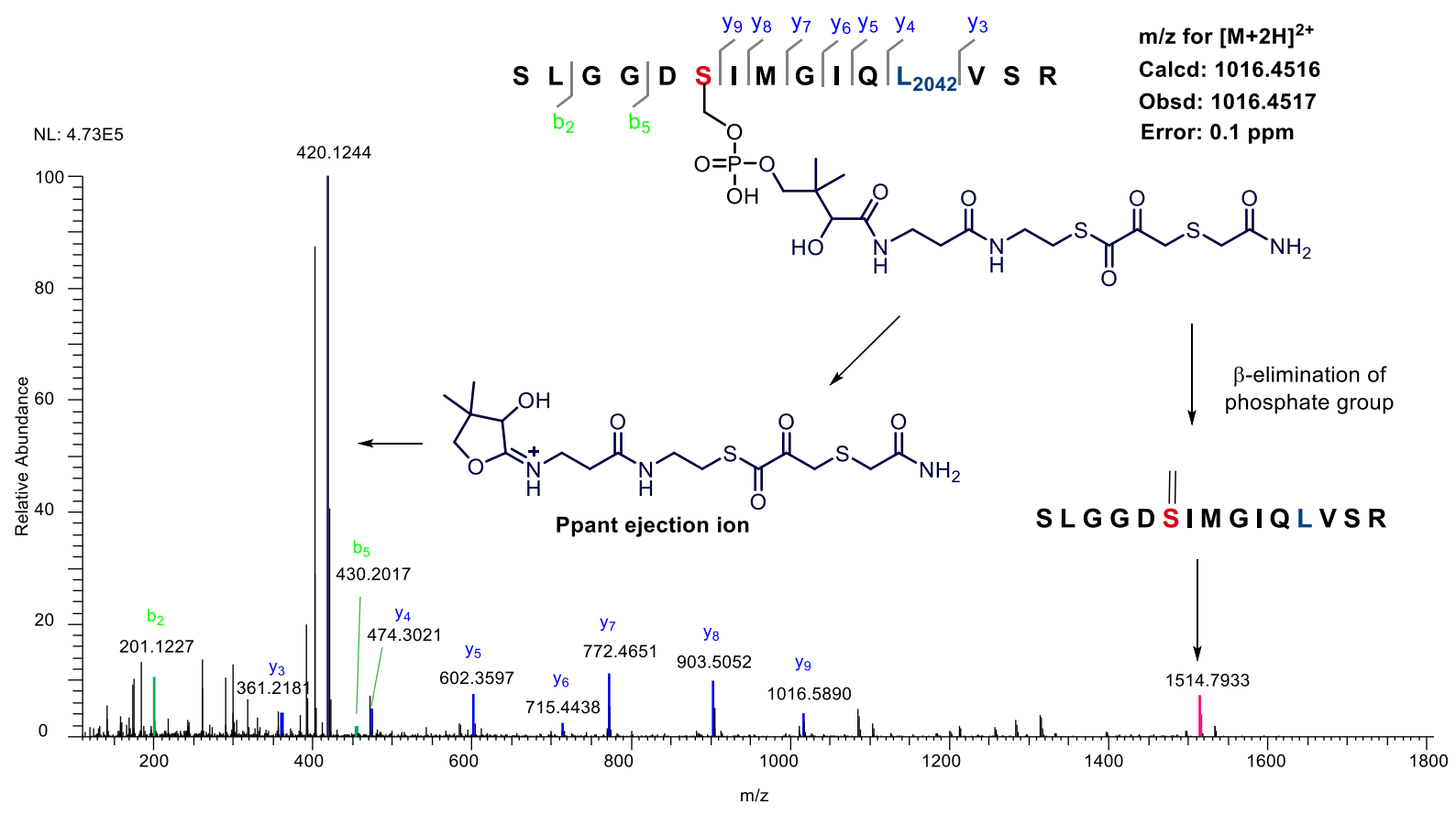

(d) MS comparison of 4 (i) and 5 (ii) in their associated Ppant ejection products, using L-cysteine (top),

$\mathrm{L}-\left[1,2,3-{ }^{13} \mathrm{C}_{3},{ }^{15} \mathrm{~N}\right]$ cysteine (middle) and L-[2,3,3-D $]$ cysteine (down) as the substrates, respectively.

d

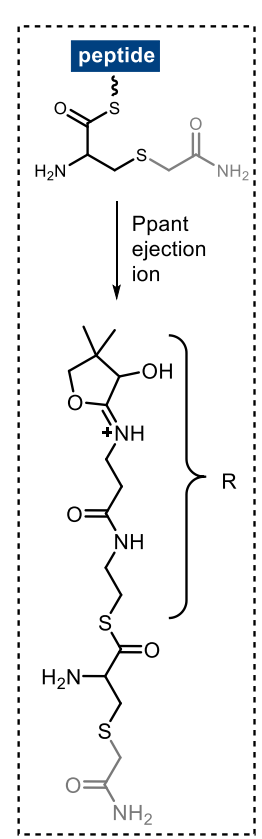

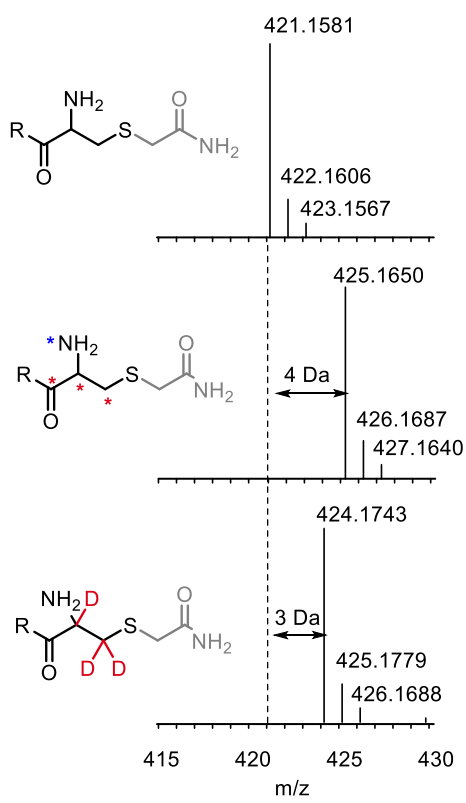

ii
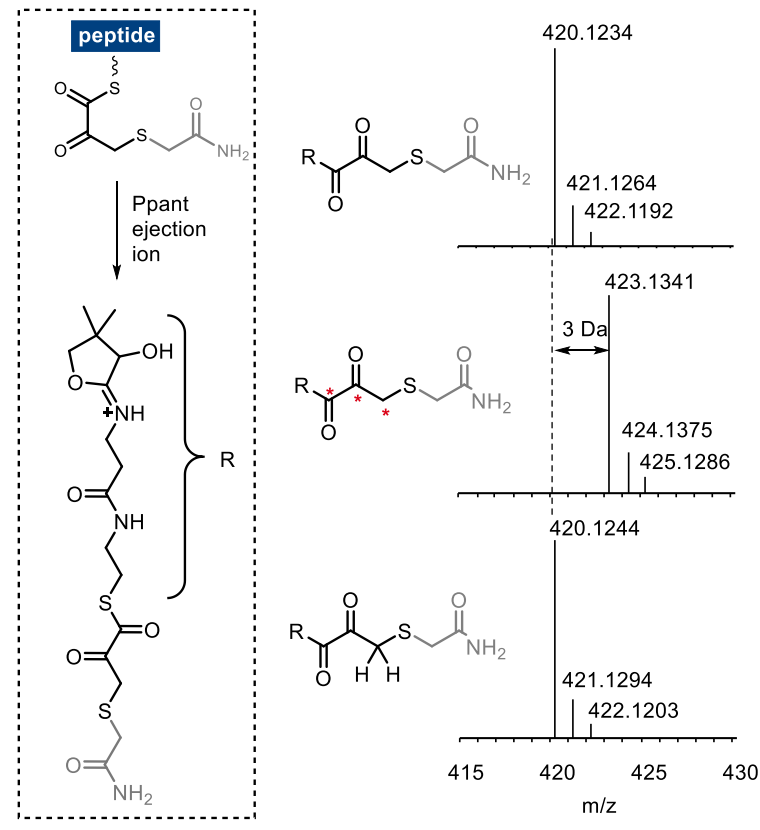
Supplementary Figure 7. Proposed mechanisms for recycling FAD in the CAEs (a) and COLs (b) biosynthetic pathways, respectively.

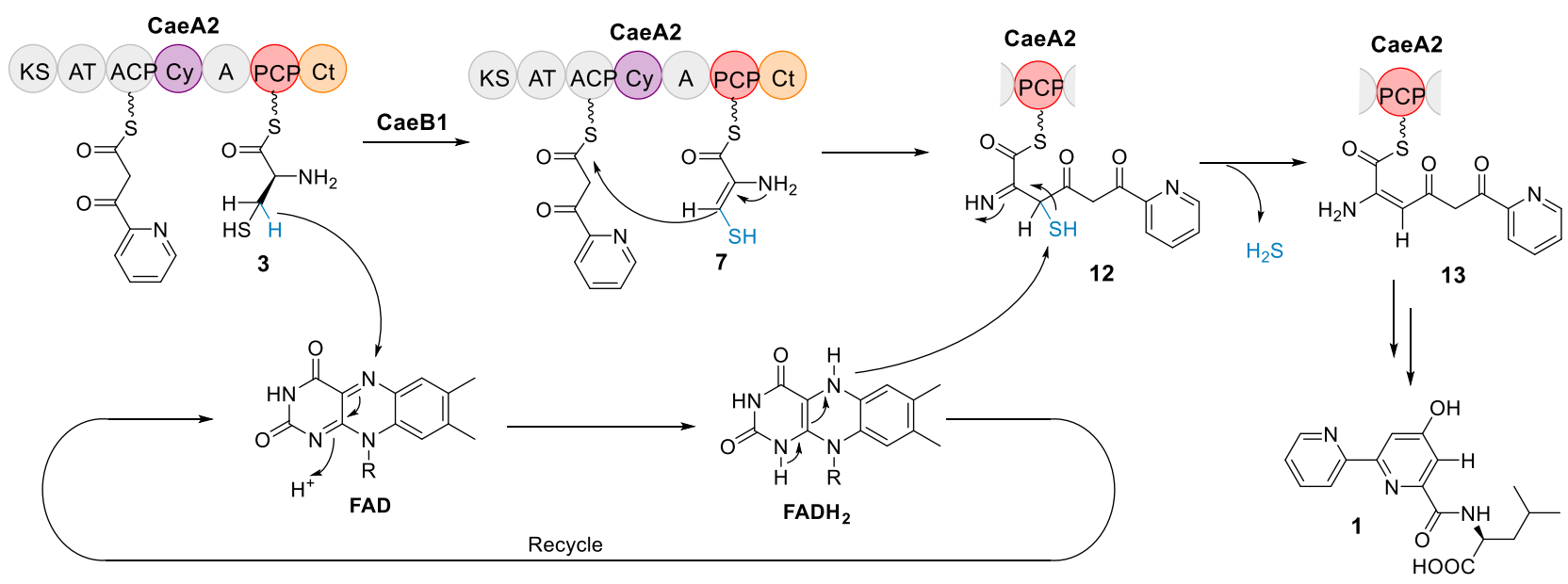

b

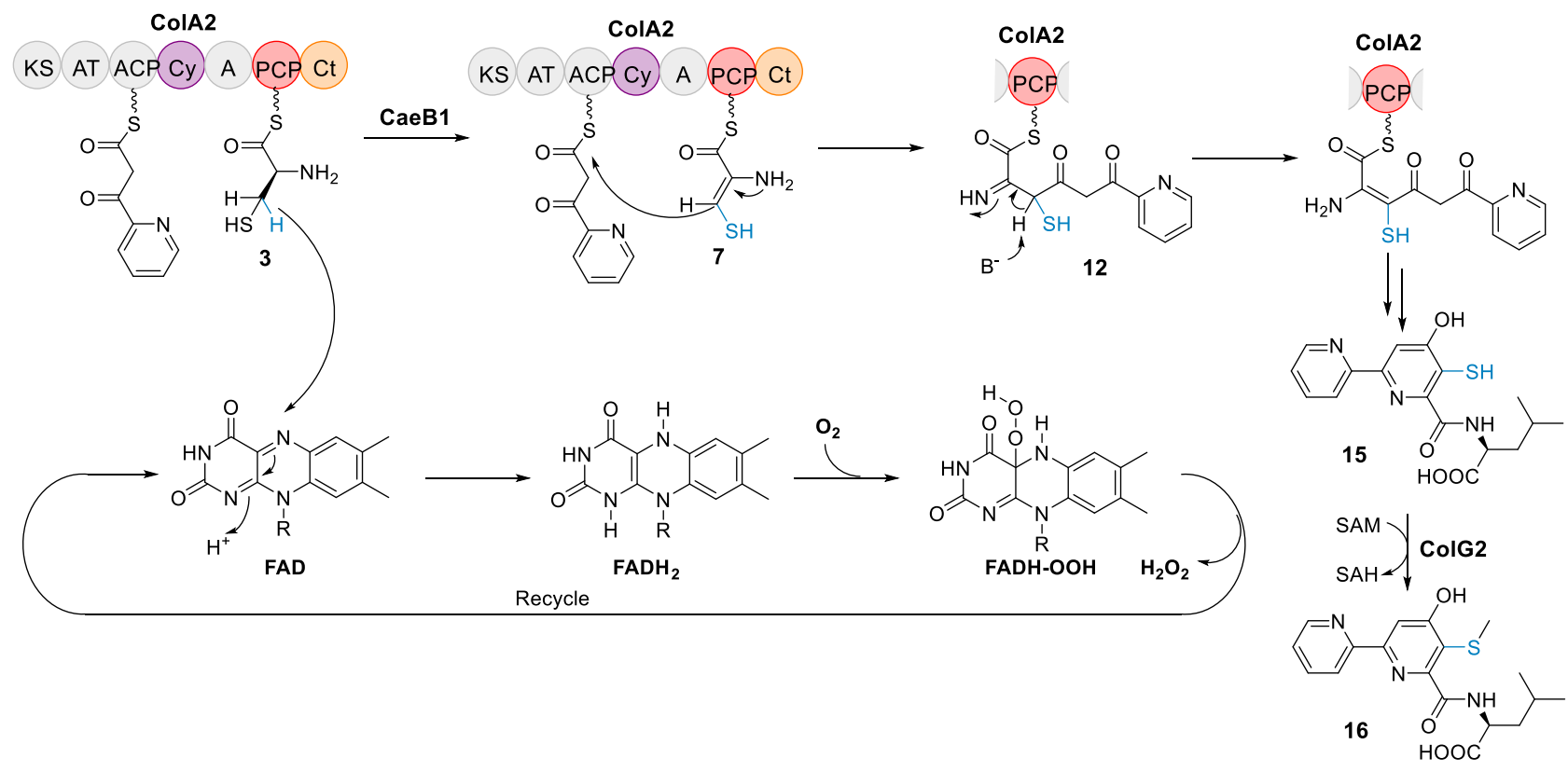


Supplementary Figure 8. Sequence analysis of the C domains of CaeA3 and ColA3, and the Cy and Ct domains of CaeA2 and ColA2 For comparison, the homologs include the epimerization (E) domain of the NRPS TycA (610-913 aa, AAC45928.1) in tyrocidine biosynthesis ${ }^{12}$, the first $\mathrm{C}$ domain of the NRPS BacB (72-361 aa, AAC06347.1) in bacitracin biosynthesis ${ }^{13}$, the C domain of the NRPS MycC (855-1156 aa, AAF08797.1) in mycosubtilin biosynthesis ${ }^{14}$, and the Cy domain of the NRPS EpoB (71-364 aa, ADB12489.1) in epothilone biosynthesis ${ }^{15}$. The boundaries of each domain were determined by Pfam. The active site residue L-histine of $\mathrm{C}$ domains is indicated by red star, and the active site residue L-glutamic acid of Cy domains is indicated by black dot. 
bioRxiv preprint doi: https://doi.org/10.1101/2020.12.07.415471; this version posted December 8, 2020. The copyright holder for this preprint (which was not certified by peer review) is the author/funder. All rights reserved. No reuse allowed without permission.

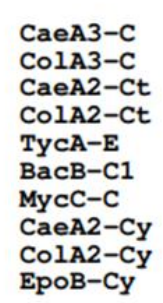

EроB-Cy

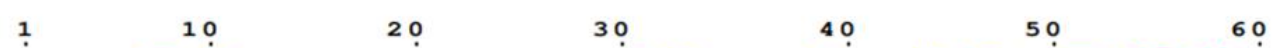

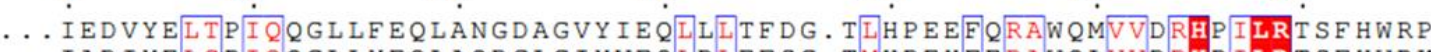

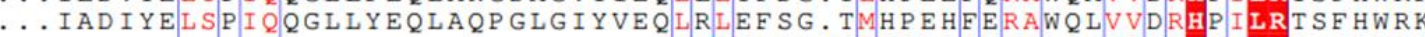

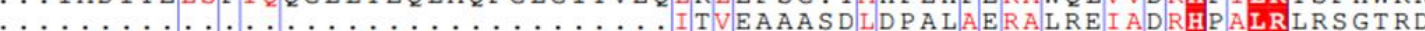

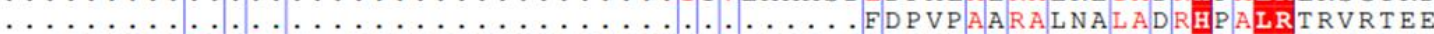
... IA GNVP L T P I K. . WFFGKNFTNTGHWNQS SVI YRPE. GE D R KVIQSVMDKI IEHHDALRMVYQHEN ....KSGTYP L REQKRMF I LNQLDDSKTAYNMP L AVK I NG. EVQ I SR L E Q WKALIKRHESLRTSFVMLD

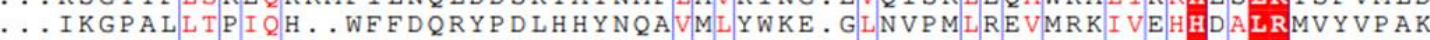
$\ldots . . Q P F P L N E M Q Q A Q W I$ GRLGSF DMGGVAPH L Y EF DSD T LAARLQRAWQQVVRRHEMLRIVVLADG EPFPITEMOQAOWIGRLSSFDMGGVAPH Y Y EFDSRTIETARIERAWORVVORHDMLRMVVLPDG $\dot{P} \dot{A} \dot{E} \dot{R} H V P F P$ L I I GSYWLGRTGAFTVPSGI. H A R EYDCADLDVARLSRAFRKVVARHDMLRAHTLPDM

70

80

90

100

110

120

130

CaeA3-C ColA3-C ColA2-Ct TYCA-E BacB-C1 MycC-C CaeA2-Cy ColA2-Cy EpoB-Cy

EGLPLQVVH. Q QHAELPLEVIDWRHIPVAEQKEKITEWLDRERVEGFDVTQAPVMRLSVIHSEPETWIFA

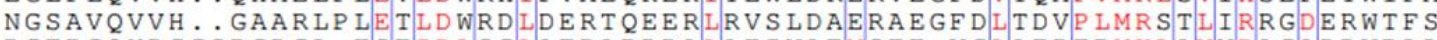
DGTPGQYDGGSPGDGL. F SEIDLSGLAEDARDDALAEIVAEMSEE.VGLAEREPVKLAVVRAGADDVRLI GQRFAVRPG..PGEDFDVPEIDLAALPDAVRAA AVEEMIGEMARE. LDIETGPAVKFAVFRLGERGSVLA GNVVQHNRGL. GGQLYDFF Y Y T AQPD. . VQQA IEAETQRLHSS. MNLQEGPLVKVALFQTLHGDHLF. GEPVQKIE... QEAEFRLEYSEL....... GDQS I QEK I SRF IKP. FEI EKAPLLRAEIVIVDEAEHMMM

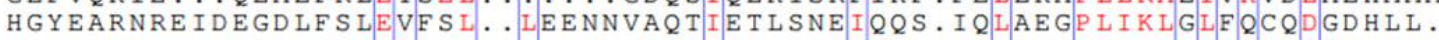
RQRILD......NVP DYEFEELD LRGVDPEEARALIAEVSDRMSTEVRPADTWP LWEVRVSRLGDGRLRTH RQQILH..... DAEPYRFEVLDLRTTDPEESERQLAGIRDRMATEVRPADVWPLWEVRVSLLPDHRVRVH MQVIE...... PKVDADIEIIDLRGLDRSTREARLVSLRDAMSHRI Y T ERPELYHVVAVRLDERQTRLV

140

150

160

170

180

190

CaeA3-C ColA3-C CaeA2-Ct ColA2-Ct TyCA-E BacB-C1 MycC-C CaeA2-Cy ColA2-Cy EpoB-Cy

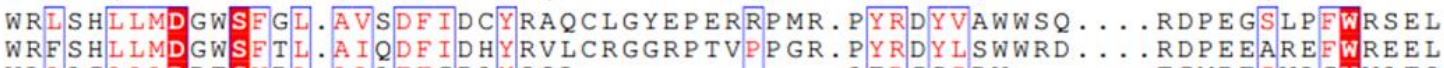

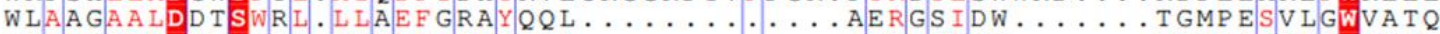

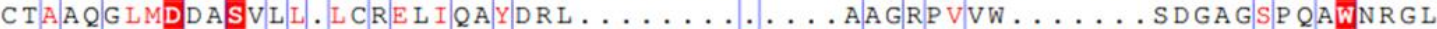
LA I H H LVVDG ISWRI. LFE D LATGYAQA LA GQA I S L E KTDSFQSWSQWLQEYANEADLLSEIPYWES LE VDMH I I SDGVSIGI. LMKEFA D CCEGK... ELS . P PAV.QYKDY SEWQRD I EQQSRLKKQEAYWLNTE IVA H H L IDGVSWR I. L I E I A A A Y ELLNGEA I Q L KKTDSYL LWAEQLKRYAESPEFEMKNQYWFQH. I SF DL L CADVASFFYQI LP SWREFHENP... ELPSEPPEL. SFRDYVLAEEALHDSPLYERSLEYWRERV I SFDLLVADVSSFFYQL LPQWREFYHHP... DRDPEPLAL, SFRDYVLAEEELRRTPRYERSLEYWRKRV LS I D L I NVDLGSLSI]. I FKDWLSFYEDP.... ETSLPVLELSYRDYVLALESRKKSEAHQRSMDYWKRRI

200 210 220 230 240 250 260

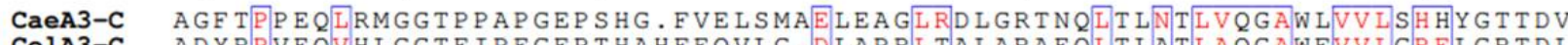

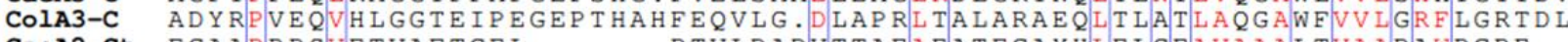
CaeA2-Ct EGAAPPPSVETVAFTGEL.........RTVLDAVTTAFAEATEGAYHLELEAVAAALTVAARAWPGRE. . ColA2-Ct RR.KPAHPAGLADTPGTPGELPRRRTVELDATRTADLFTAAAGSHHIDPTEVLVAAASTLGR. SQAKNVSLPKDYEVTDCKQKSVRNMRIRLHPEETEQLLKHANQAYQTEINDLLLAALGLAFAEWSKLAQI TYCA-E $\mathrm{BacB}-\mathrm{C} 1$ MycC-C CaeA2-Cy EpoB-Cy RGD I PVLNMP L D F RPK IRSFQGNRTVVELDQDT TKKLKT I A K NGVTMYMLLLA GYTILLSKYTGQEDI EH I P P K L KDNEQEIGLAEDRETI IVQWTAEETERLLKNAHRAYTTEMNDLLLTGLGIAIHRWTGHEDI

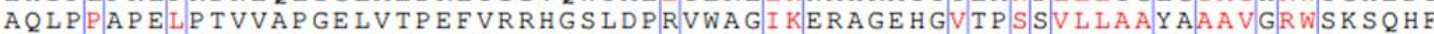
RELPAAPELPTVQGAGGGERLGFVRRHARLDAEPWGRIKAKAGEFGVTPSSAMLAAFAVTIGTSKSQRE

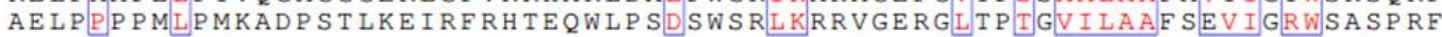


Supplementary Figure 9. Characterization of L-Cysteinyl-S-CaeA2 ${ }^{\mathrm{F} 2042 \mathrm{~L}} \triangle \mathrm{Ct}(\mathbf{8})$ by nanoLC-MS/MS following treatment with IAM and subsequent complete digestion with trypsin and chymotrypsin.

(a) Incubation of truncated $\mathrm{CaeA} 2^{\mathrm{F} 2042 \mathrm{~L}} \triangle \mathrm{Ct}$ and L-cysteine.

a

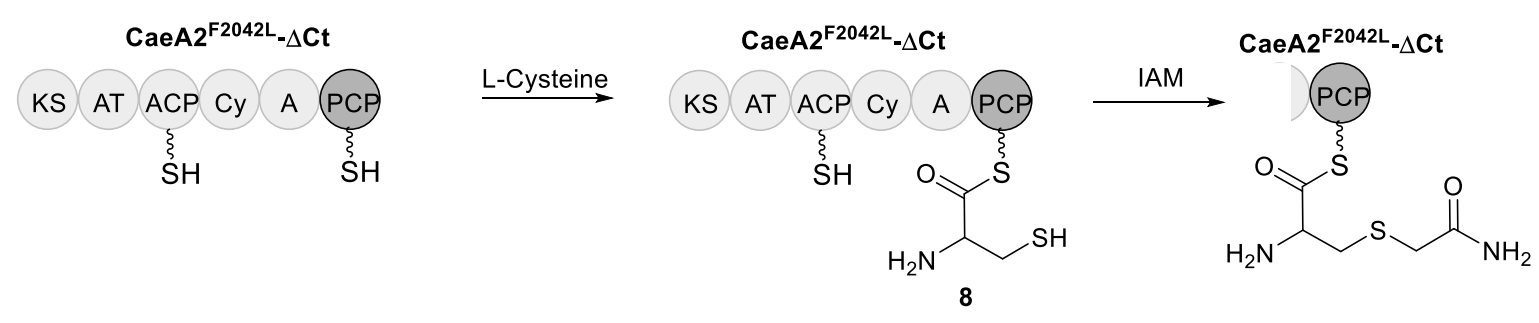<smiles>NC(=O)CSCC(N)C(=O)Sc1ccccc1</smiles>

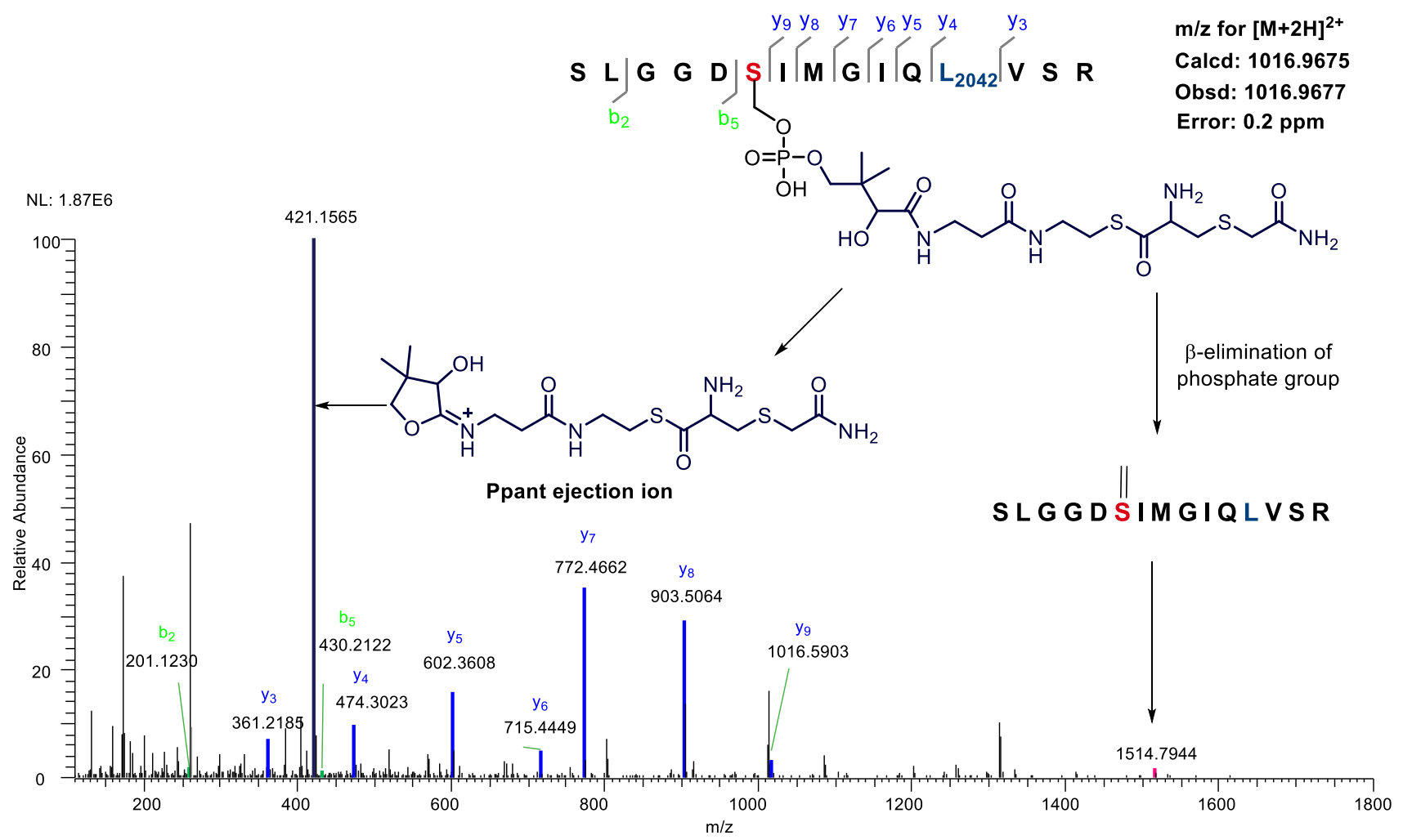

(b) Incubation of CaeB1 with truncated CaeA2 $2^{\mathrm{F} 2042 \mathrm{~L}} \triangle \mathrm{Ct}$ and L-cysteine. 
b

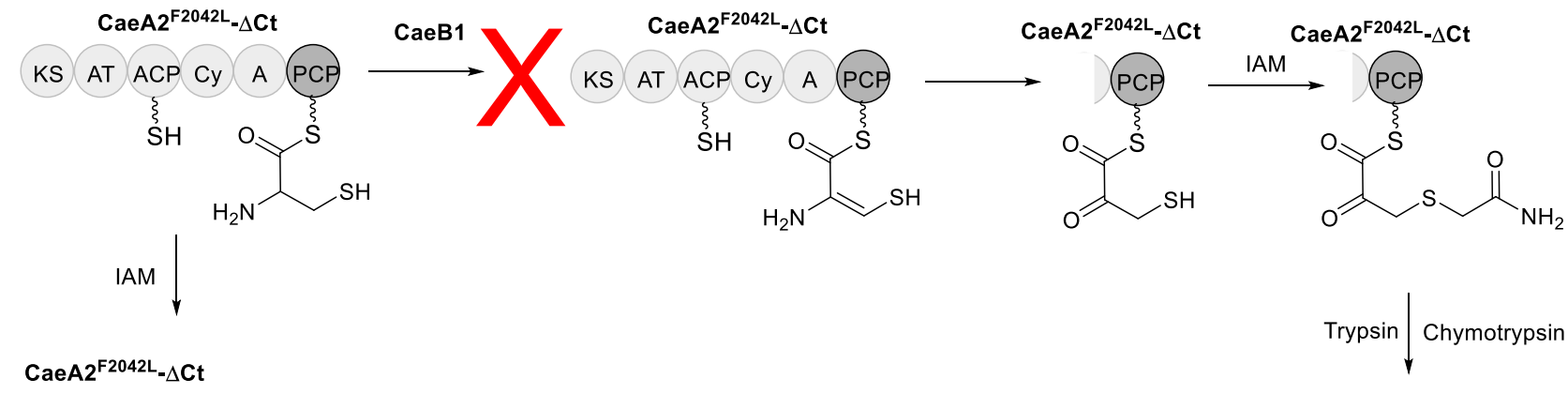

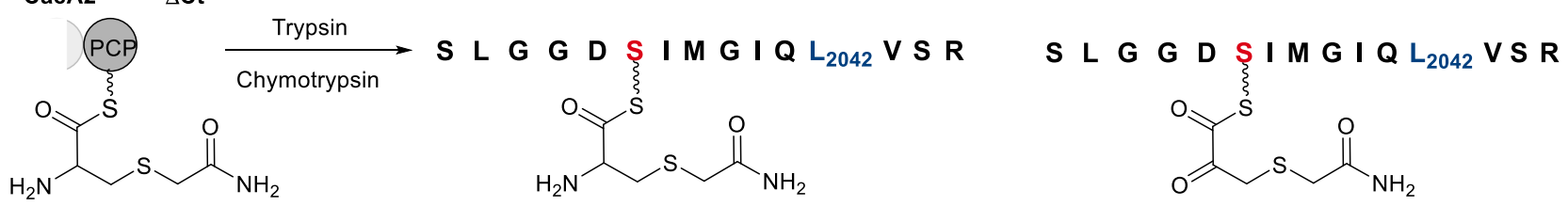

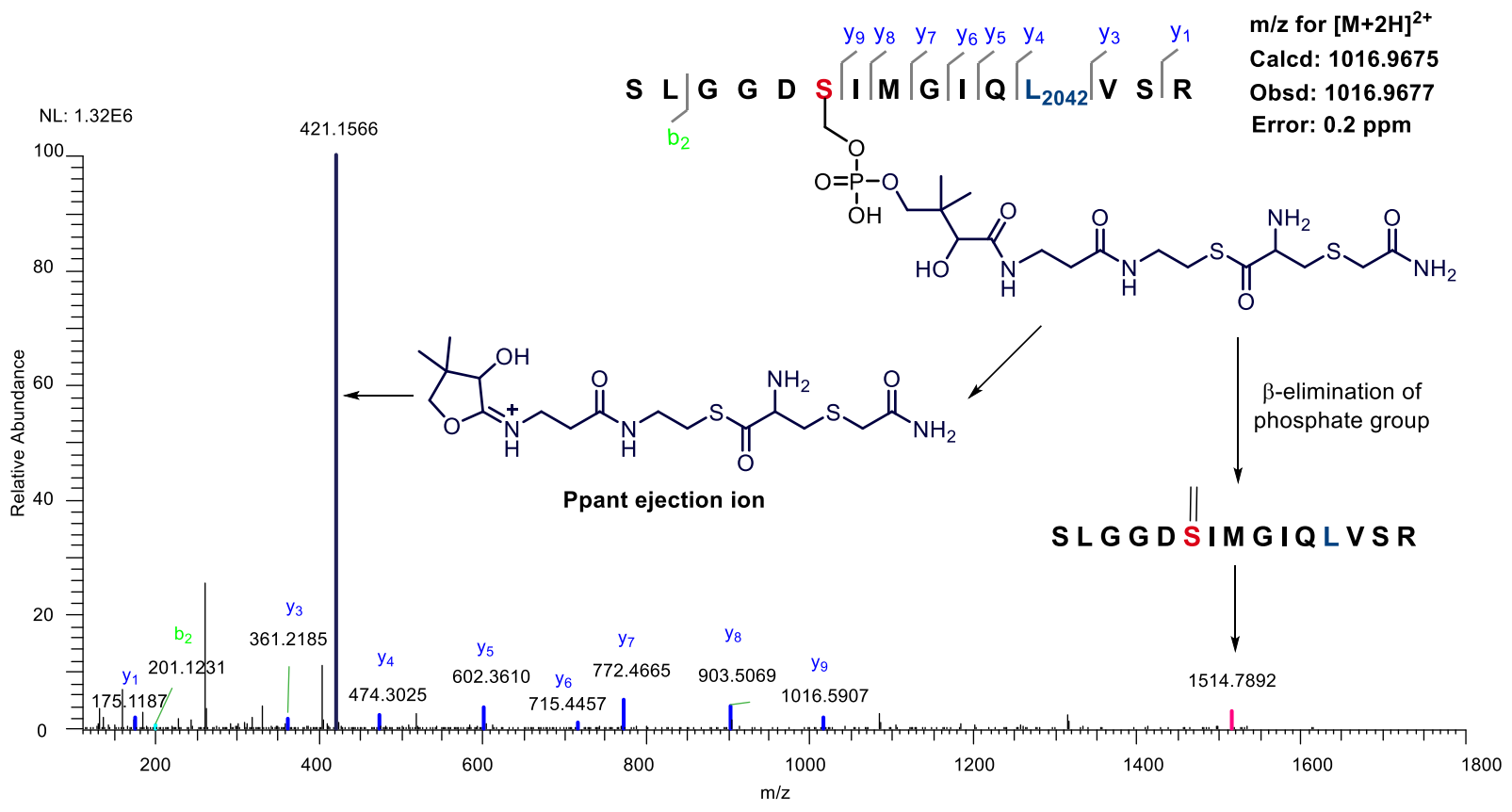


Supplementary Figure 10. Measurement of the interactions of truncated CaeA2- $\Delta \mathrm{Ct}$ with related flavoproteins by ITC. Raw dates were shown on top, and the integrated curves containing experimental points and the best fitting line obtained from the single binding site model were shown on bottom. (a) Titrating MBP-fused CaeB1 to CaeA2- $\Delta$ Ct. (b) Titrating ColB1 to CaeA2- $\Delta$ Ct.
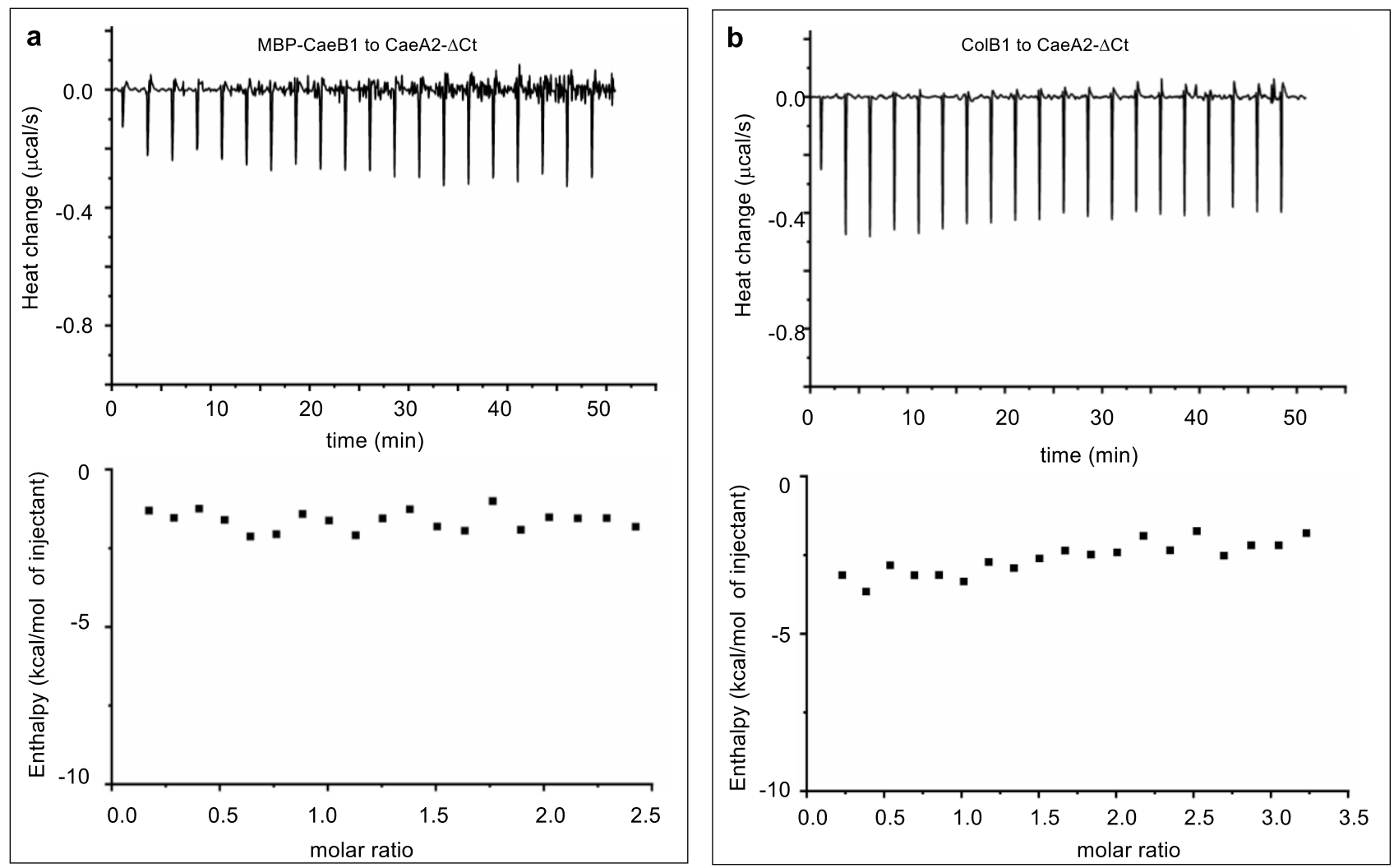
Supplementary Figure 11. Characterization of 2,2'-bipyridinyl-S-CaeA2 $2^{\mathrm{F} 2042 \mathrm{~L}}$ (9) by nanoLCMS/MS following complete digestion with trypsin and chymotrypsin.

(a) Incubation of CaeA1, CaeA2 $2^{\mathrm{F} 2042 \mathrm{~L}}$ and $\mathrm{CaeB} 1$ with picolinic acid, malonyl-S-CoA and L-cysteine.

a

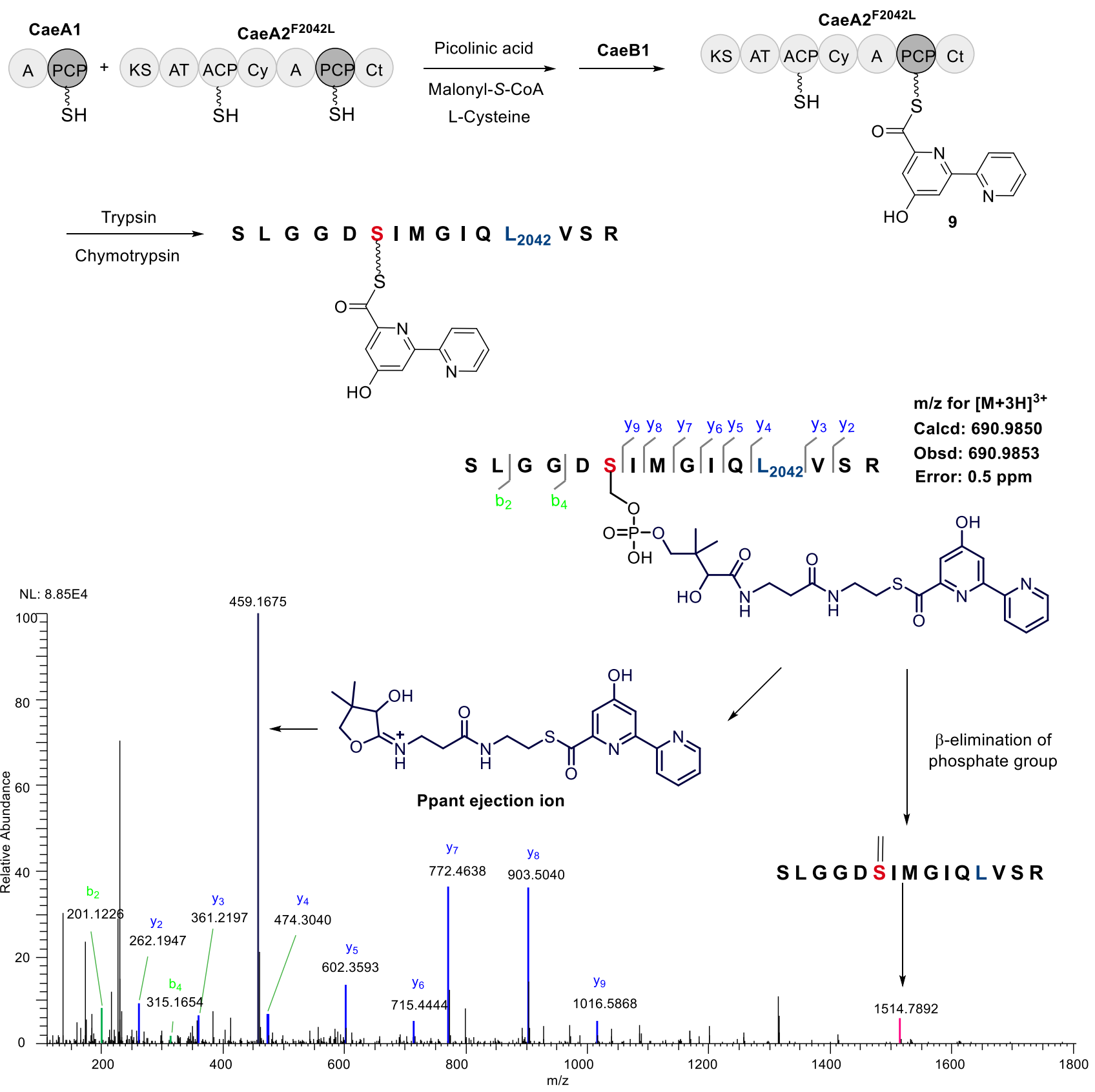


(b) Incubation of Ppant-unmodified CaeA2 ${ }^{\mathrm{F} 2042 \mathrm{~L}}$ (produced in E. coli BL21(DE3)) with synthesized 2,2'-bipyridinyl-S-CoA in the presence of Sfp.

b
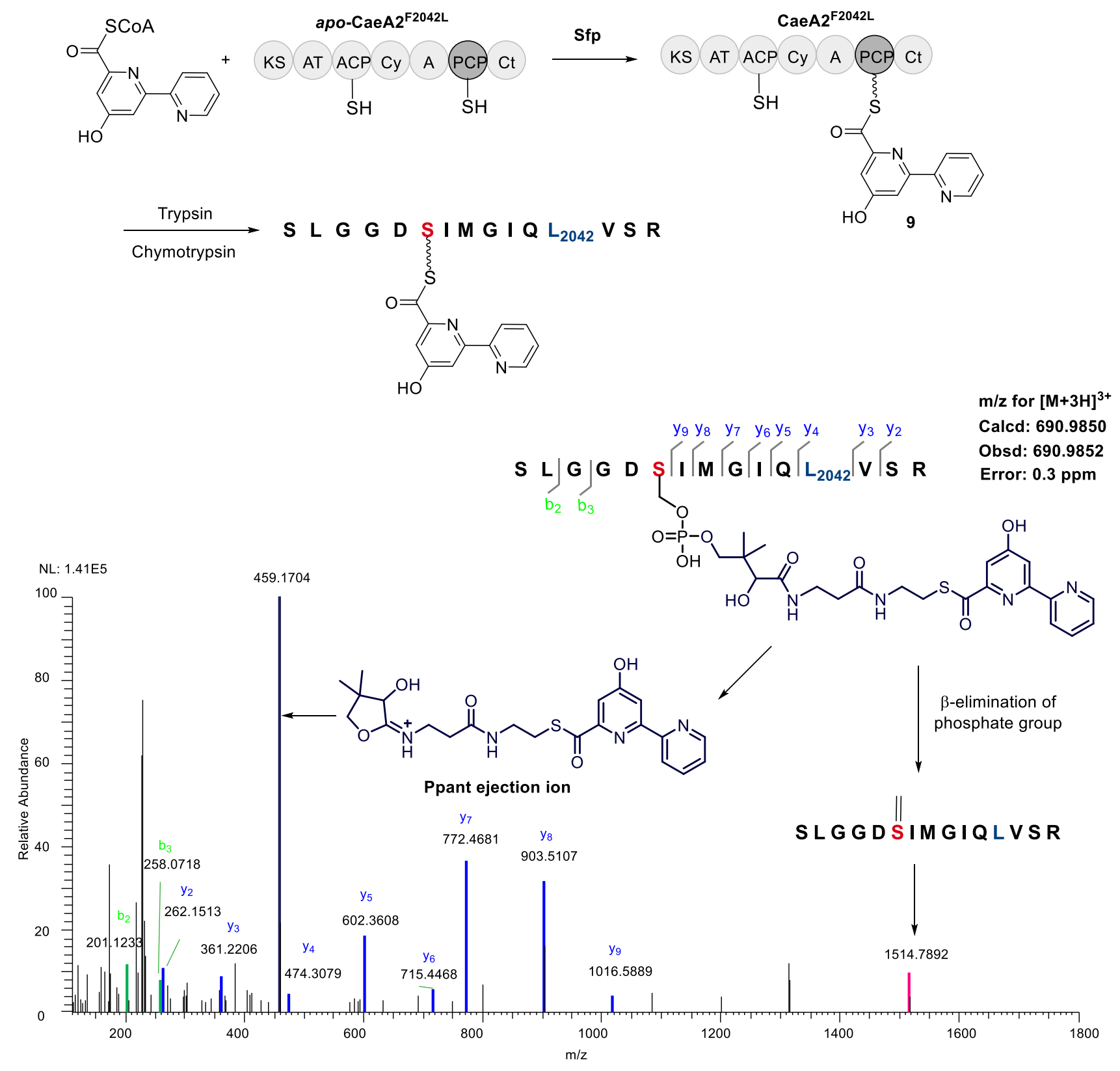
Supplementary Figure 12. Validation of 2,2'-bipyridinyl-S-Ppant as an intermediate in the production of 1. (a) Preparation of 2,2'-bipyridinyl-S-PCP $\mathrm{CaeA}_{2}(\mathbf{1 0})$ by the incubation of $\mathrm{PCP}_{\mathrm{CaeA} 2}$ in apo form (produced in E. coli BL21(DE3)) with synthesized 2,2'-bipyridinyl-S-CoA in the presence of Sfp. $\mathrm{PCP}_{\mathrm{CaeA} 2}$ in both apo (i) and holo (ii) forms and 2,2'-bipyridinyl-S-PCP $\mathrm{CaeA}_{2}$ (iii) were examined by HR-MS. (b) Incubation of prepared 2,2'-bipyridinyl-S-PCP ${ }_{\mathrm{CaeA} 2}$ with CaeA3 and L-leucine in the absence (i) and presence (ii) of ATP, the standard of 1 (iii).

a

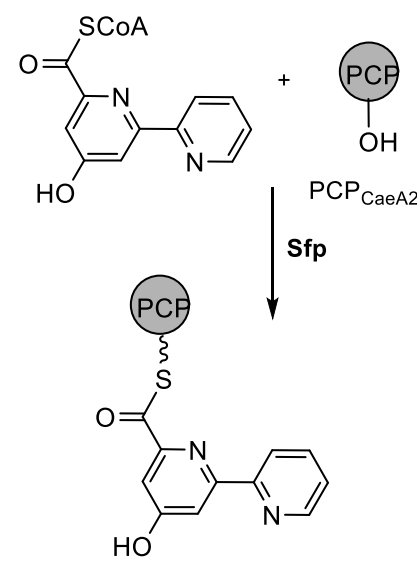

2,2'-bipyridinyl-S-PCP $\mathrm{CaeA}_{2}$ (10)

b

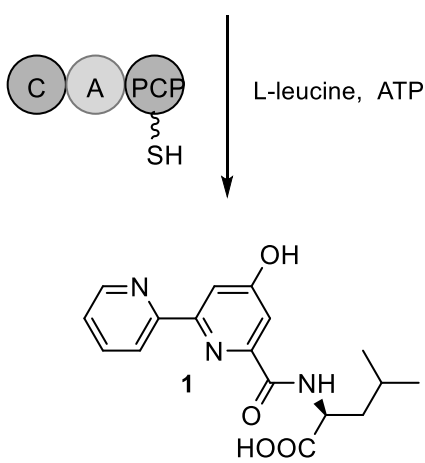

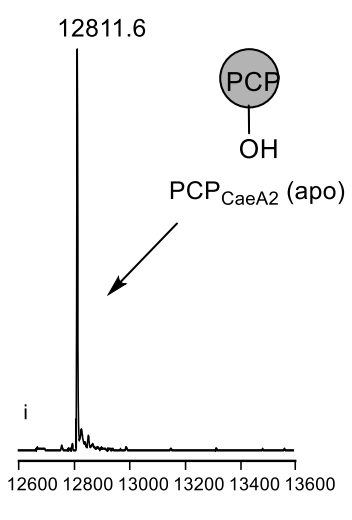
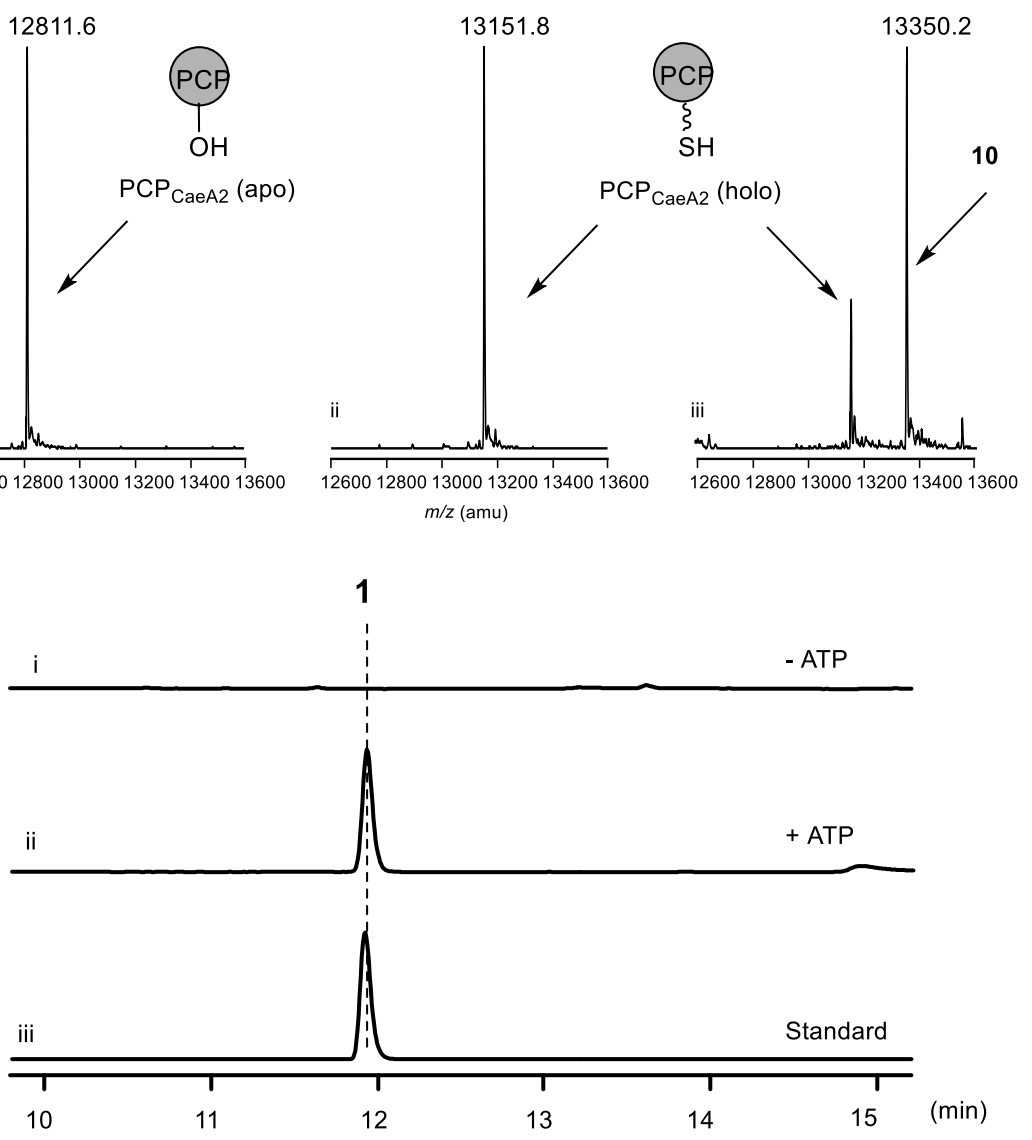
Supplementary Figure 13. Phylogenetic analysis of the $\mathrm{C}$ domains of NRPS CaeA3 and ColA3 in substrate stereo-chemistry. The evolutionary distances were computed using the p-distance method. The support for grouping the clades ${ }^{\mathrm{L}} \mathrm{C}_{\mathrm{L}}$ (green) and ${ }^{\mathrm{D}} \mathrm{C}_{\mathrm{L}}$ (blue) is indicated by bootstrap value. The $\mathrm{C}$ domains of CaeA3, ColA3 and the $\mathrm{C}$ domain of NocB that mediates $\beta$-lactam formation in nocardicin biosynthesis ${ }^{16}$ are shown in red. The homologous $\mathrm{C}$ domains arise from the NRPSs BA1, BA2 and BA3 in bacitracin biosynthesis ${ }^{13}$; CdaPS1, CdaPS2 and CdaPS3 in calciumdependent antibiotic biosynthesis ${ }^{17}$; ComB, ComC and ComD in complestatin biosynthesis ${ }^{18}$; Fen1, Fen2, Fen3, Fen4 and Fen5 in fengycin bioysynthesis ${ }^{19}$; GrsB in gramicidin biosynthesis ${ }^{20}$; ItuA, ItuB and ItuC in iturin biosynthesis ${ }^{21}$; Lic A, LicB and LicC in lichenicin biosynthesis ${ }^{22}$; MycA, MycB and MycC in mycosubtilin biosynthesis ${ }^{23}$; SnaE1 and SnaE2 in pristinamycin biosynthesis ${ }^{24}$; and TycB and TycC in tyrocidine biosynthesis ${ }^{12}$. The sequences were downloaded from the database of NaPDoS, in which the biochemical function and substrate stereo-chemistry of these related C domains have been confirmed ${ }^{25,26}$.

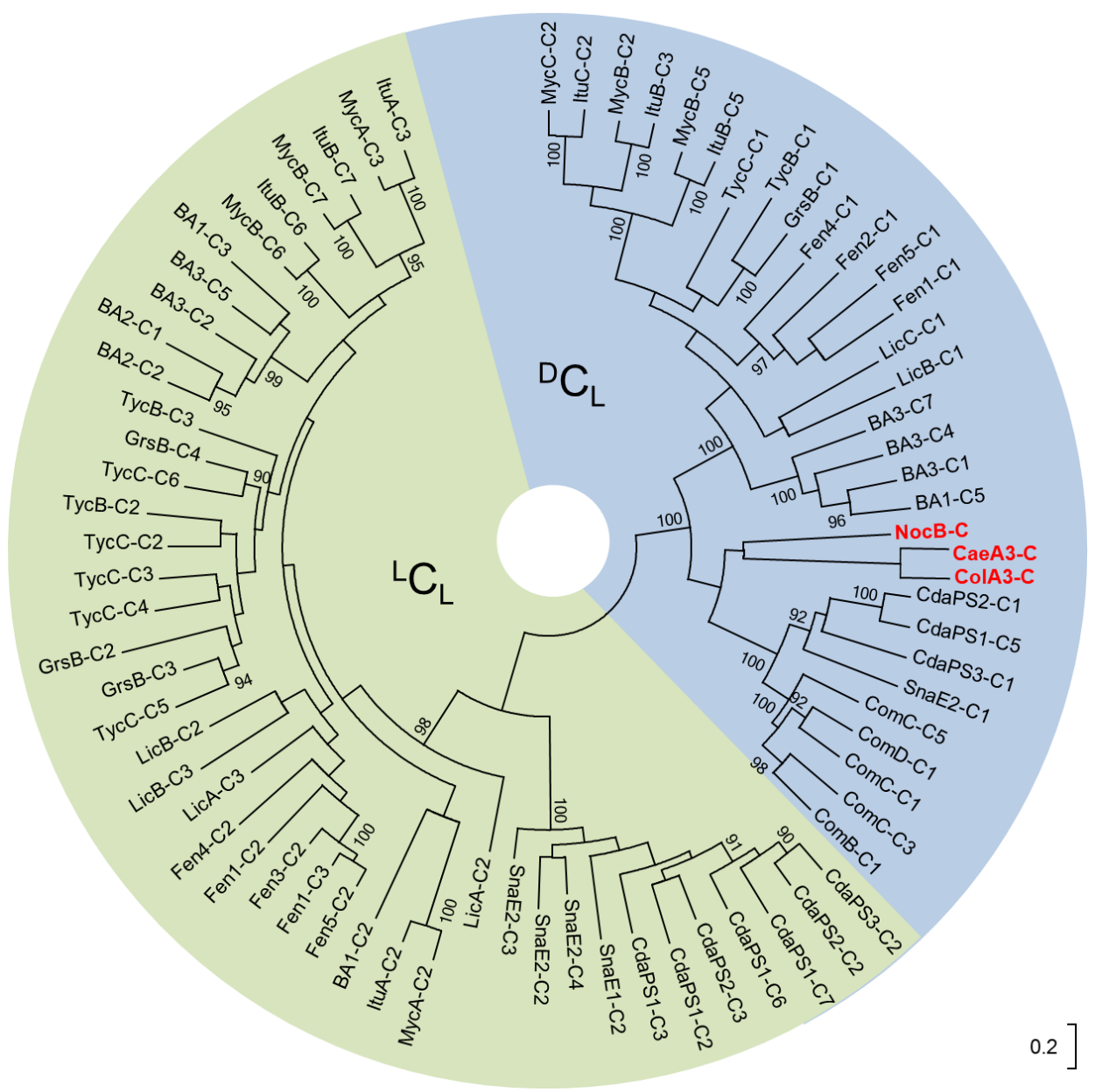


Supplementary Figure 14. Phylogenetic analysis of the Cy domains of the NRPSs CaeA2 and ColA2. The evolutionary distances were computed using the p-distance method. The support for grouping the $\mathrm{C}$ domain subtypes is indicated by bootstrap value. The Cy domains of CaeA2 and ColA2 are shown in red. Members of the $\mathrm{C}$ domain subtypes $\left({ }^{\mathrm{L}} \mathrm{C}_{\mathrm{L}},{ }^{\mathrm{D}} \mathrm{C}_{\mathrm{L}}\right.$, Starter $\mathrm{C}$, Epimerization (E), Duel E/C and Heterocyclization (Cy) domains) include those of the NRPSs Act2 and Act3 in actinomycin biosynthesis $^{27}$; ArfA and ArfB in arthrofactin biosynthesis ${ }^{28}$, Arg3 in argyrins biosynthesis ${ }^{29}$; BA1 and BA2 in bacitracin biosynthesis ${ }^{13}$; BmdB in bacillamide biosynthesis ${ }^{30}$; BlmVIII and BlmIV in bleomycin biosynthesis ${ }^{31}$; ClbJ and $\mathrm{ClbK}$ in colibactin biosynthesis ${ }^{32}$; CdaPS1 in calcium-dependent antibiotic biosynthesis ${ }^{17}$; EpoP in epothilone biosynthesis ${ }^{15}$; EntF in enterobactin biosynthesis ${ }^{33}$; Fen1, Fen2 and Fen3 in fengycin bioysynthesis ${ }^{19}$; Grs A and GrsB in gramicidin biosynthesis ${ }^{20}$; HMWP2 in yersiniabactin biosynthesis ${ }^{34}$; ItuB in iturin biosynthesis ${ }^{21}$; LicA and LicB in lichenicin biosynthesis ${ }^{22}$; McyA and McyB in microcystin biosynthesis ${ }^{35}$; MycB in mycosubtilin biosynthesis ${ }^{23}$; PchB and PchC in pyochelin biosynthesis ${ }^{36}$; RamB in ramoplanin biosynthesis ${ }^{37}$; SrfA and SrfB in surfactin biosynthesis ${ }^{38}$; SypA, SypB and SypC in syringopeptin biosynthesis ${ }^{39}$; $\mathrm{StaC}$ in A47934 biosynthesis ${ }^{40}$; SyrA, SyrB and SyrE in syringomycin biosynthesis ${ }^{41}$; TycB and TycC in tyrocidin biosynthesis ${ }^{12}$; TioR in thiocoraline biosynthesis ${ }^{42}$; VibH in vibriobactin biosynthesis ${ }^{43}$. The sequences were downloaded from the database of $\mathrm{NaPDoS}$, in which the biochemical function of these related $\mathrm{C}$ domains have been characterized $^{25,26}$. 


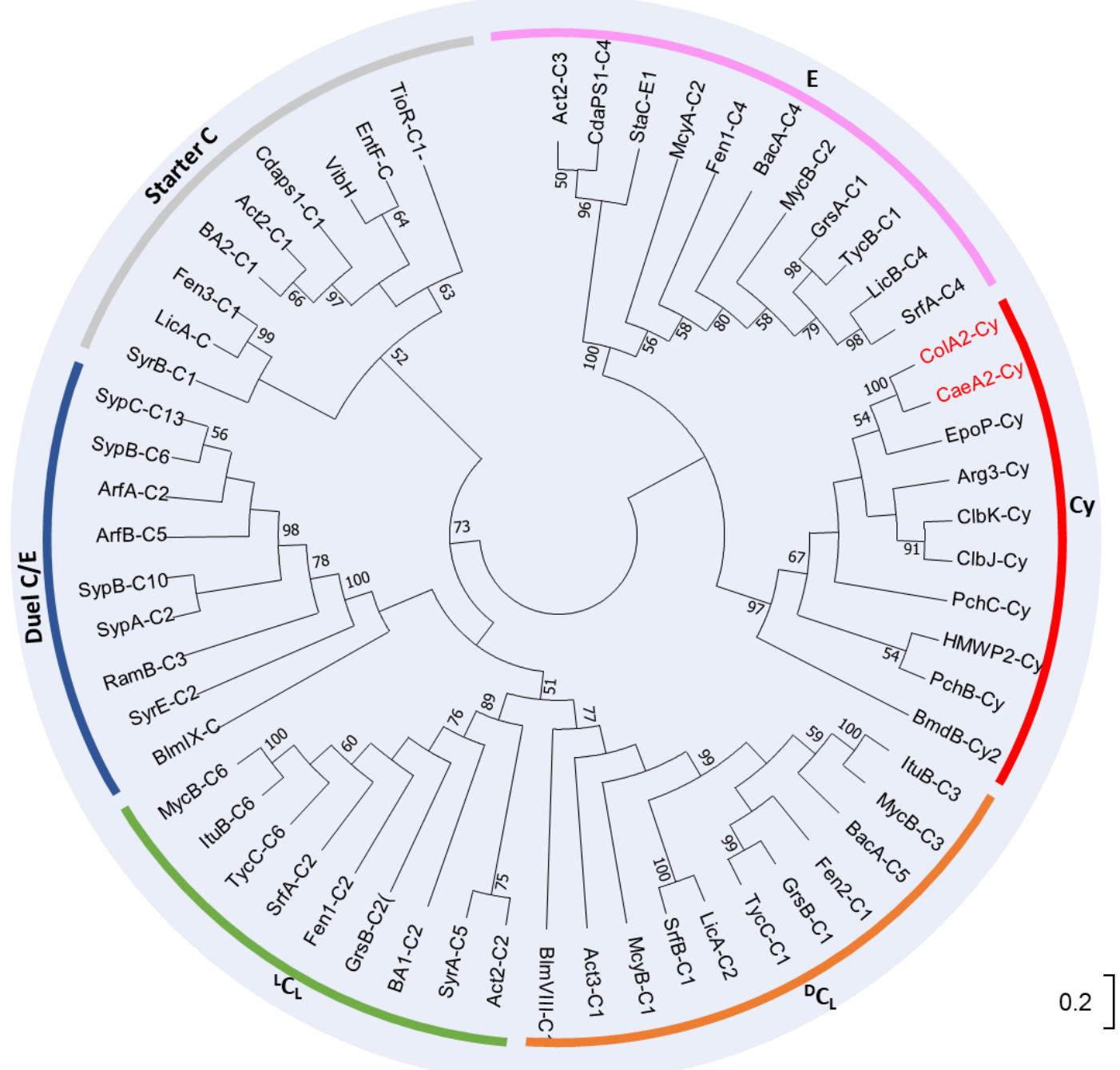


Supplementary Figure 15. Comparison in the production of the CAE 2,2'-bipyridine intermediate 1 using the substrate L-cysteine (i), $\mathrm{L}-\left[1,2,3-{ }^{13} \mathrm{C}_{3},{ }^{15} \mathrm{~N}\right]$ cysteine (ii) and $\mathrm{L}-\left[2,3,3-\mathrm{D}_{3}\right]$ cysteine (iii), respectively. (a) HPLC analysis. (b) HR-MS analysis.

a

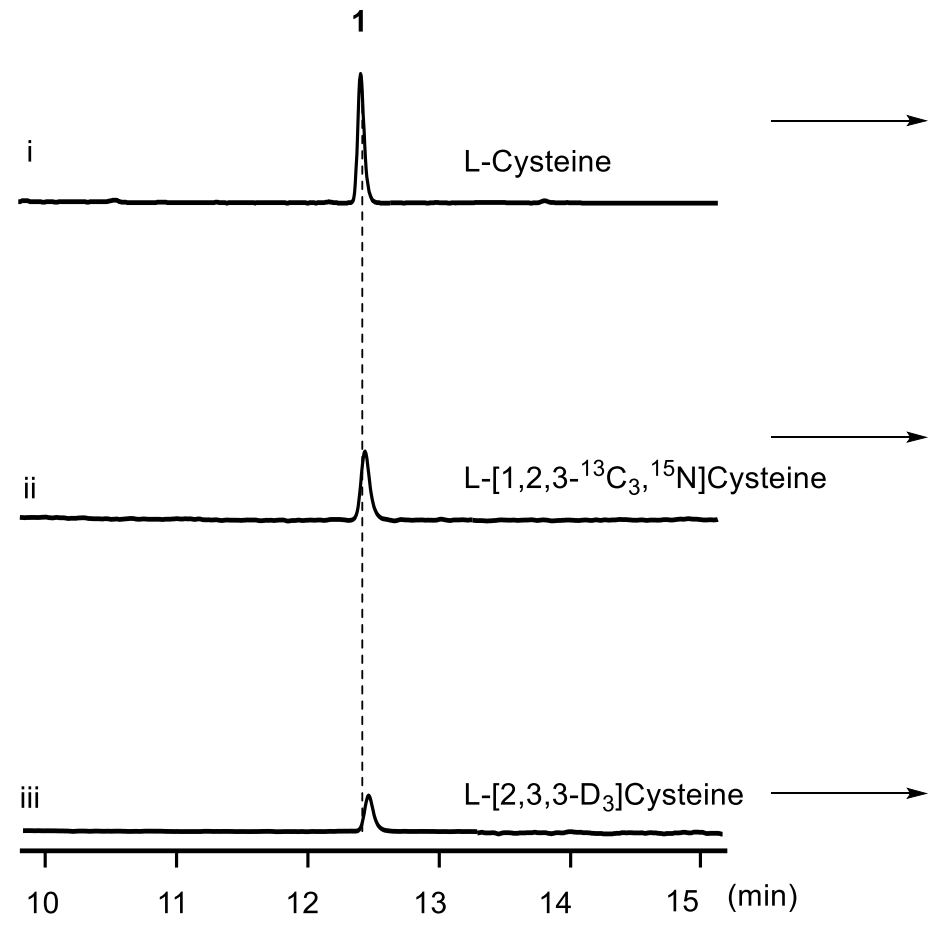

b

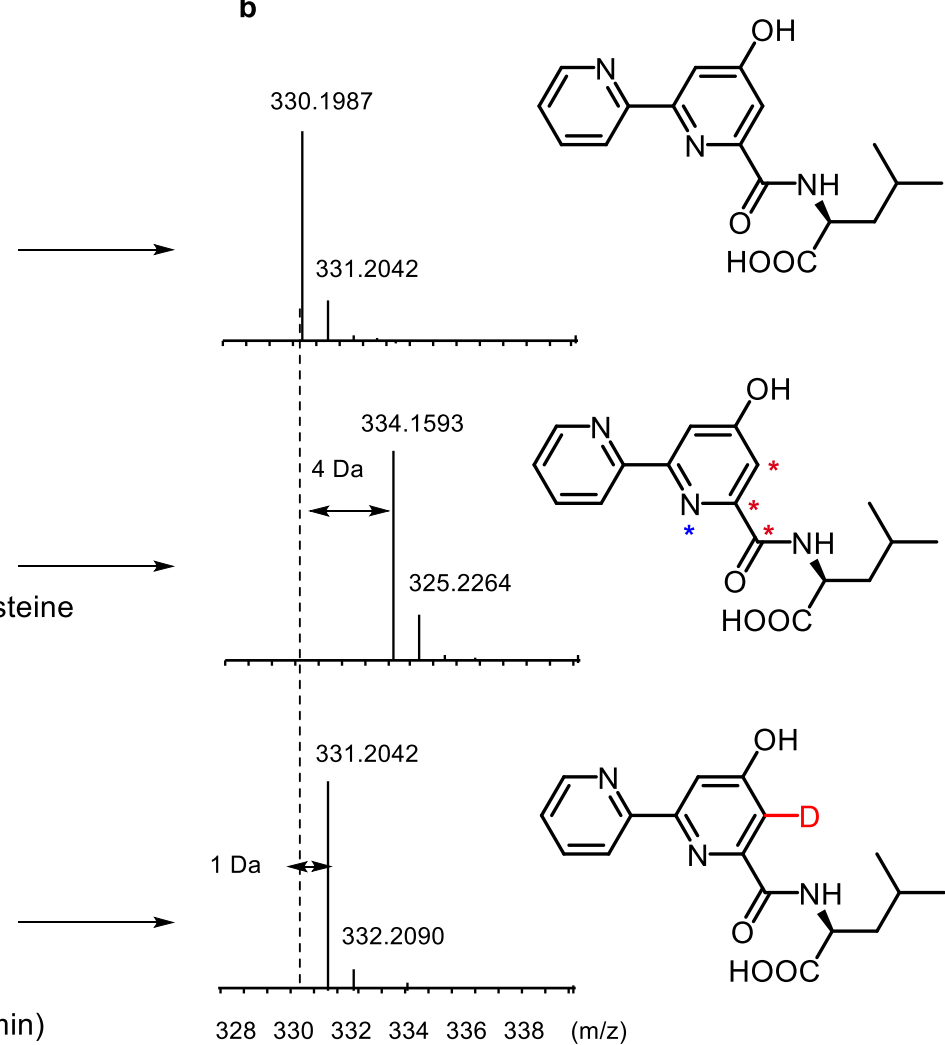


Supplementary Figure 16. Examination of thiol intermediate 5-sulfhydryl-2,2'-bipyridinyl-L-leucine (15) by IAM derivatization. 15 was produced in the reactions where CaeA1, CaeA2, CaeB1and ColA3 were combined with picolinic acid, malonyl-S-CoA, L-cysteine and L-leucine in the presence (i) or absence (ii) of ATP. The reaction mixtures were treated with IAM to produce 17, which were then analyzed by HPLC (left, $\lambda=315 \mathrm{~nm}$ ) and HR-MS (right, $[\mathrm{M}+\mathrm{H}]^{+} \quad m / z$ : cald. 419.1389 for $\mathrm{C}_{19} \mathrm{H}_{22} \mathrm{O}_{5} \mathrm{~N}_{4} \mathrm{~S}$, obs. 419.1370).
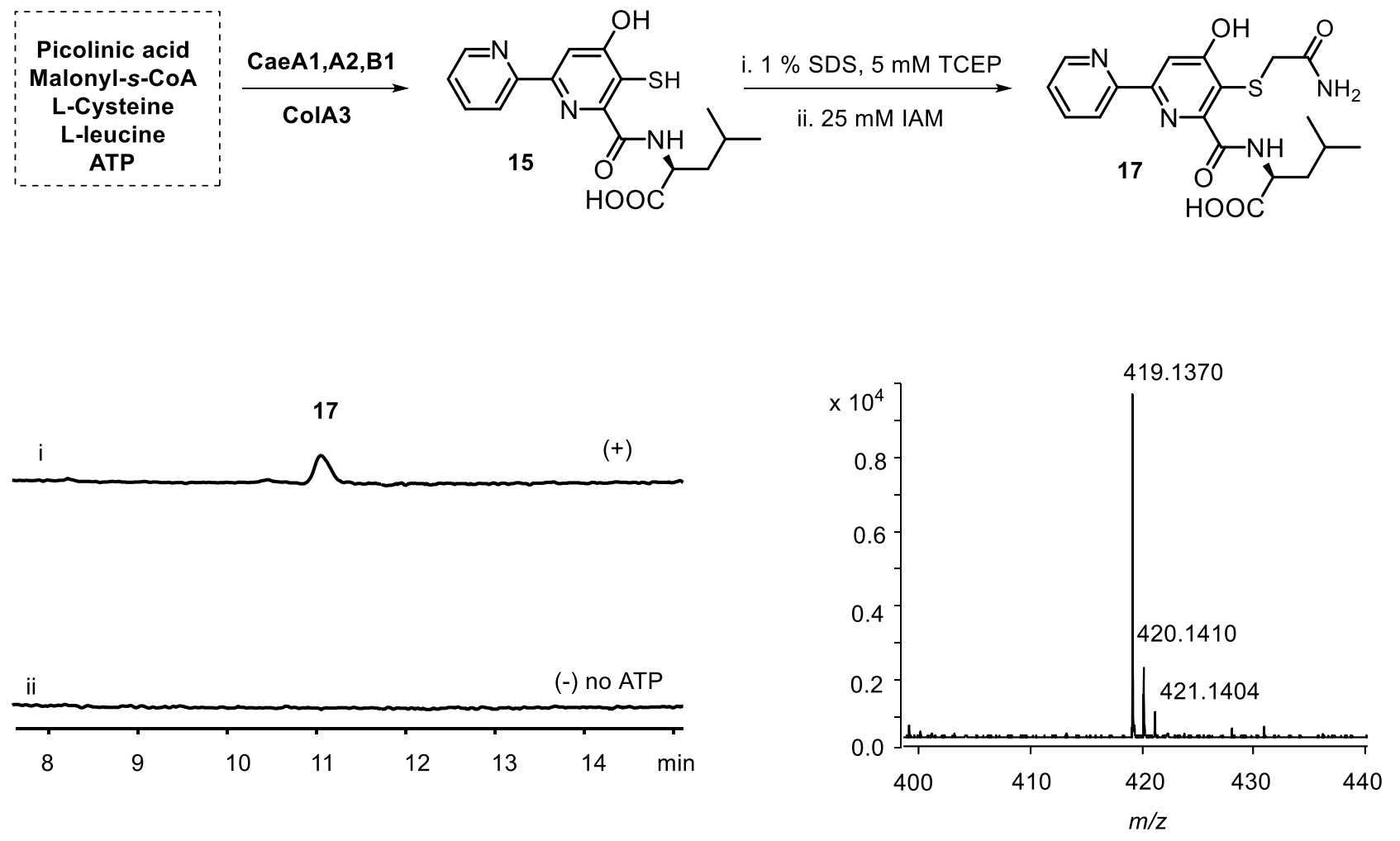


\section{SUPPLEMENTARY TABLES}

Table S1. Bacterial strains in this study.

\begin{tabular}{|c|c|c|}
\hline Strain/plasmid & Characteristics & Source \\
\hline \multicolumn{3}{|l|}{ E. coli } \\
\hline DH5 $\alpha$ & Host for general cloning & Invitrogen \\
\hline S17-1 & $\begin{array}{l}\text { Donor strain for conjugation between E. coli and } \\
\text { Streptomyces }\end{array}$ & 2 \\
\hline BL21 (DE3) & Host for protein expression & NEB \\
\hline BAP1 & $\begin{array}{l}\text { Host for protein expression, contains } s f p \text { gene under the } \\
\text { control of the T7 RNA polymerase promoter }\end{array}$ & 44 \\
\hline \multicolumn{3}{|l|}{ S. raseosporus } \\
\hline NRRL 11379 & COL-producing wild type strain & NRRL \\
\hline QL2003 & colA2 inframe deletion mutant, COL non-producing & 7 \\
\hline QL2006 & $\begin{array}{l}\text { QL2003 derivative, in which chimeric gene col/caeA2 was } \\
\text { expressed in trans }\end{array}$ & This study \\
\hline \multicolumn{3}{|l|}{ A. cyanogriseus } \\
\hline NRRL B-2194 & CAE-producing wild type strain & NRRL \\
\hline
\end{tabular}


Table S2. Plasmids in this study.

\begin{tabular}{|c|c|c|}
\hline Plasmids & Characteristics & Source \\
\hline pMD19-T & E. coli subcloning vector, ampicillin resistrance & Takara \\
\hline pSET152 & $\begin{array}{l}\text { E. coli-Streptomyces shuttle vector for gene } \\
\text { complementation, apramycin resistance }\end{array}$ & 2 \\
\hline $\mathrm{pET}-28 \mathrm{a}(+)$ & $\begin{array}{l}\text { Protein expression vector used in E.coli, encoding N- } \\
\text { terminal } 6 \times \text { His-tag, kanamycin resistance }\end{array}$ & Novagen \\
\hline $\mathrm{pET}-37 \mathrm{~b}(+)$ & $\begin{array}{l}\text { Protein expression vector used in E.coli, encoding C- } \\
\text { terminal } 8 \times \text { His-tag, kanamycin resistance }\end{array}$ & Novagen \\
\hline pSJ5 & $\begin{array}{l}\text { Protein expression vector used in E.coli, encoding N- } \\
\text { terminal His } \times 6 \text {-Trx-tag, ampicillin resistance }\end{array}$ & $\begin{array}{l}\text { Transformed from } \\
\text { pET32a, adding Trx- } \\
\text { tag and after N- } \\
\text { terminal His }\end{array}$ \\
\hline pQ8 & $\begin{array}{l}\text { Protein expression vector used in E.coli, encoding N- } \\
\text { terminal His } \times 6 \text {-MBP-His } \times 6 \text {-tag, kanamycin resistance }\end{array}$ & 45 \\
\hline pQL1022 & $\begin{array}{l}\text { pSET152 derivative containing the } 8.0 \mathrm{~kb} \text { ermE* } \\
\text { promoter }+ \text { colA } 2 \text { gene }\end{array}$ & 7 \\
\hline pQL1026 & pMD19-T derivative, containing caeAl & This study \\
\hline pQL1027 & $\mathrm{pET}-28 \mathrm{a}(+)$ derivative, containing caeAl & This study \\
\hline pQL1028 & pMD19-T derivative, containing caeA2 & This study \\
\hline pQL1029 & pET-37b $(+)$ derivative, containing caeA2 & This study \\
\hline pQL1030 & pMD19-T derivative, containing caeA3 & This study \\
\hline PQL1031 & pET-28a $(+)$ derivative, containing caeA3 & This study \\
\hline pQL1032 & pMD19-T derivative, containing $c a e B 1$ & This study \\
\hline pQL1033 & pET-28a $(+)$ derivative, containing $c a e B 1$ & This study \\
\hline pQL1034 & pMD19-T derivative, containing colB 1 & This study \\
\hline PQL1035 & pET-28a $(+)$ derivative, containing colB 1 & This study \\
\hline pQL1036 & pMD19-T derivative, containing colA3 & This study \\
\hline
\end{tabular}




\begin{tabular}{|c|c|c|}
\hline pQL1037 & pET-28a $(+)$ derivative, containing colA3 & This study \\
\hline pQL1038 & pMD19-T derivative, containing caeA4 & This study \\
\hline pQL1039 & pET-28a $(+)$ derivative, containing ceaA4 & This study \\
\hline pQL1040 & pMD19-T derivative, containing caeA2- $\Delta C t$ & This study \\
\hline PQL1041 & $\mathrm{pET}-28 \mathrm{a}(+)$ derivative, containing $c a e A 2-\Delta C t$ & This study \\
\hline pQL1042 & pMD19-T derivative, containing colG2 & This study \\
\hline pQL1043 & pET-28a $(+)$ derivative, containing colG2 & This study \\
\hline pQL1044 & $\begin{array}{l}\text { pMD19-T derivative, containing the gene encoding the } \\
\text { PCP domain of CaeA2 }\end{array}$ & This study \\
\hline pQL1045 & $\begin{array}{l}\text { pET-28a }(+) \text { derivative, containing the gene encoding } \\
\text { the PCP domain of CaeA2 }\end{array}$ & This study \\
\hline pQL1046 & $\begin{array}{l}\text { pMD19-T derivative, the gene encoding the } \mathrm{C} \text { domain } \\
\text { of CaeA3 }\end{array}$ & This study \\
\hline pQL1047 & $\begin{array}{l}\text { pMD19-T derivative, containing the gene encoding the } \\
\text { A-PCP didomain of ColA3 }\end{array}$ & This study \\
\hline pQL1048 & $\begin{array}{l}\text { pET-28a }(+) \text { derivative, containing the gene encoding } \\
\text { chimeric NRPS Cae/ColA3 }\end{array}$ & This study \\
\hline pQL1049 & $\begin{array}{l}\text { pET-37b }(+) \text { derivative, containing the gene encoding } \\
\text { CaeA2-F2042L }\end{array}$ & This study \\
\hline pQL1050 & $\begin{array}{l}\text { pET-37b }(+) \text { derivative, containing the gene encoding } \\
\text { CaeA2-F2042I }\end{array}$ & This study \\
\hline pQL1051 & $\begin{array}{l}\text { pET-37b }(+) \text { derivative, containing the gene encoding } \\
\text { CaeA2-F2042V }\end{array}$ & This study \\
\hline pQL1052 & $\begin{array}{l}\text { pET-37b }(+) \text { derivative, containing the gene encoding } \\
\text { CaeA2-F2042L- } \Delta \text { Ct }\end{array}$ & \\
\hline pQL1053 & $\begin{array}{l}\text { pMD19-T derivative, containing the gene encoding the } \\
\text { NRPS module of CaeA2 }\end{array}$ & This study \\
\hline
\end{tabular}




\begin{tabular}{|l|l|l|}
\hline pQL1054 & $\begin{array}{l}\text { pQL1022 derivative, containing the chimeric gene } \\
\text { col/caeA2 coding for the hybrid protein that harbors the } \\
\text { PKS module from ColA2 and the NRPS module from } \\
\text { CaeA2 }\end{array}$ & This study \\
\hline pQL1055 & $\begin{array}{l}\text { pSJ5 derivative, containing the gene encoding the Ct } \\
\text { domain of CaeA2 }\end{array}$ & This study \\
\hline pQL1055 & pQ8 derivative, containing the gene encoding CeaB1 & This study \\
\hline
\end{tabular}

Table S3. Primers used in this study. The sequences of restriction enzymes are underlined.

\begin{tabular}{|l|l|}
\hline Primer & Sequence \\
\hline CaeA1-For & 5'-GCGCGAATTCCATATGGAAGAACTGCGAAGGATT-3' (NdeI) \\
\hline CaeA1-Rev & 5'-ATATAAGCTTACTCGAGGGTTCGGGAAGCGTCC-3' (HindIII) \\
\hline CaeA2-For & 5'-AGCTGAATTCCATATGACCGGTCATGACACCGT-3' (NdeI) \\
\hline CaeA2-Rev & $\begin{array}{l}\text { 5'-AGCTAAGCTTACTCGAGCTTCTCAAGGGTGGACAGC-3' } \\
\text { (XhoI) }\end{array}$ \\
\hline CaeA3-For & $\begin{array}{l}\text { 5'-GCGCGAATTC } \text { 'AATATGAGCCAGTTGAAAAAGGGCATCG-3' } \\
\text { ('NdeI) }\end{array}$ \\
\hline CaeA3-Rev & 5'-ATATAAGCTTACTCGAGCGCCCGCTCCCTCTCCAC-3' (HindIII) \\
\hline CaeA4-For & 5'-GCGCGAATTCCATATG TTCAGGTCGTGGTCGGCGG-3'(NdeI) \\
\hline CaeA4-Rev & 5'-ATATAAGCTTTCAGGGGGACGACCGCTGCGGCG-3' (HindIII) \\
\hline CaeB1-For & 5'-ATGCGAATTCCATATGAGCGCACCGACGGTGACCAC-3' (NdeI) \\
\hline CaeB1-Rev & $\begin{array}{l}\text { 5'-ATGCAAGCTTACTCGAGCAGCGACCCCGCGATGCTCC-3' } \\
\text { ('HindIII) }\end{array}$ \\
\hline ColB1-For & $\begin{array}{l}\text { 5'-GCGCGAATTCCATATGAATACGAACCCCGGACAGAGC-3' } \\
\text { ('NdeI) }\end{array}$ \\
\hline ColA3-For & 5'-ATATAAGCTTACTCGAGGTCACCGGGGCTCCCT-3' (HindIII) \\
\hline
\end{tabular}




\begin{tabular}{|c|c|}
\hline ColA3-Rev & $\begin{array}{l}\text { 5'-TTAAGCTTACTCGAGCCGTGCCCCCTCCCCCGCGACC-3' } \\
\text { (HindIII) }\end{array}$ \\
\hline CaeA2- $\Delta$ Ct-For & 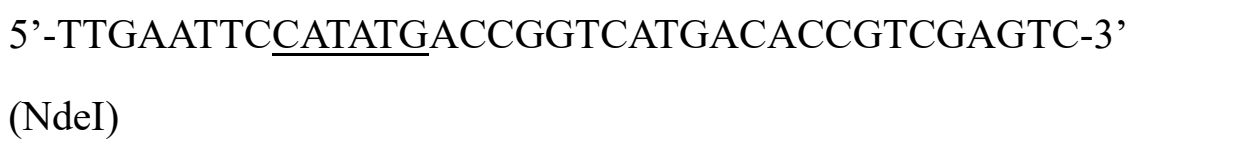 \\
\hline CaeA2- $\Delta$ Ct-Rev & $\begin{array}{l}\text { 5'-TTAAGCTTACTCGAGGGCCGCCTGGTGCGGGGTGAAC-3' } \\
\text { (XhoI) }\end{array}$ \\
\hline ColG2-For & 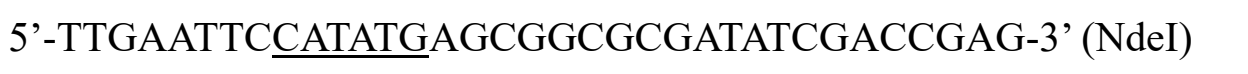 \\
\hline ColG2-Rev & 5'-TTAAGCTTCTACCCCGGTACGACGGTCTCGA-3' (HindIII) \\
\hline CaeA2-PCP-For & 5'-TTGAATTCCATATGCCCGCGCCGGACCGCGTCGG-3' (NdeI) \\
\hline CaeA2-PCP-Rev & $\begin{array}{l}\text { 5'-TTAAGCTTACTCGAGCGCGACCGTCCCGTCCGCCTC-3' } \\
\text { (HindIII) }\end{array}$ \\
\hline CaeA3-C-For & $\begin{array}{l}\text { 5'- } \\
\text { TTGAATTCCATATGAAAAAGGGCATCGAGGATGTCTACGAACTC } \\
\text { AC-3' (NdeI) }\end{array}$ \\
\hline CaeA3-C-Rev & 5'-TTGCTAGCGTCGGGGTTGGCGGCGGCCAGGTC-3' (NheI) \\
\hline ColA3-APCP-For & 5'-TTGCTAGCGCCGTGCGCTGCGGAGGCCG-3' (NheI) \\
\hline ColA3-APCP-Rev & $\begin{array}{l}\text { 5'-TTAAGCTTACTCGAGCCGTGCCCCCTCCCCCGCGAC-3' } \\
\text { (HindIII) }\end{array}$ \\
\hline CaeA2-F2042L-For & 5'-TGGGTATCCAGTTGGTCAGCCGTGCCG-3' \\
\hline $\begin{array}{l}\text { CaeA2-F2042L- } \\
\text { Rev }\end{array}$ & 5'-GGCTGACCAACTGGATACCCATGATCGA-3' \\
\hline CaeA2-F2042I- For & 5'-TGGGTATCCAGATCGTCAGCCGTGCCG-3' \\
\hline CaeA2-F2042I-Rev & 5'-GGCTGACGATCTGGATACCCATGATCGA-3' \\
\hline CaeA2-F2042V-For & 5'-TGGGTATCCAGGTCGTCAGCCGTGCCG-3' \\
\hline $\begin{array}{l}\text { CaeA2-F2042V- } \\
\text { Rev }\end{array}$ & 5'-GGCTGACGACCTGGATACCCATGATCGA-3' \\
\hline Cae-CO-for & 5'-TTAGATCTCAGCCGTTCCCGCTGAACGAGAT-3' (BgIII) \\
\hline Cae-Ct-rev & 5'-TTGATATCTTACTTCTCAAGGGTGGACAGCAGGGC-3' (EcoRV) \\
\hline
\end{tabular}


Table S4. MS/MS data for PCP-aminoacylation on CaeA2.

Detail MS/MS data for Figure S4A

\begin{tabular}{|c|c|c|c|}
\hline & Calcd $m / z$ & Obsd $m / z$ & Error \\
\hline $\mathrm{b} 2$ & 201.1239 & 201.1220 & $9 \mathrm{ppm}$ \\
\hline $\mathrm{y} 3$ & 361.2198 & 361.2183 & $4 \mathrm{ppm}$ \\
\hline $\mathrm{y} 4$ & 508.2882 & 508.2856 & $5 \mathrm{ppm}$ \\
\hline $\mathrm{y} 5$ & 636.3467 & 636.3420 & $7 \mathrm{ppm}$ \\
\hline $\mathrm{y} 7$ & 806.4521 & 806.4467 & $6 \mathrm{ppm}$ \\
\hline $\mathrm{y} 8$ & 937.4925 & 937.4888 & $4 \mathrm{ppm}$ \\
\hline $\mathrm{y} 9$ & 1050.576 & 1050.5739 & $2 \mathrm{ppm}$ \\
\hline Ppant ejection ion & 261.1267 & 261.1252 & $5 \mathrm{ppm}$ \\
\hline
\end{tabular}

Detail MS/MS data for Figure S4B

\begin{tabular}{|c|c|c|c|}
\hline & Calcd $m / z$ & Obsd $m / z$ & Error \\
\hline $\mathrm{b} 2$ & 201.1239 & 201.1220 & $9 \mathrm{ppm}$ \\
\hline $\mathrm{y} 3$ & 361.2198 & 361.2178 & $5 \mathrm{ppm}$ \\
\hline $\mathrm{y} 4$ & 474.3038 & 474.3040 & $0.5 \mathrm{ppm}$ \\
\hline $\mathrm{y} 5$ & 602.3623 & 602.3628 & $1 \mathrm{ppm}$ \\
\hline $\mathrm{y} 6$ & 715.4463 & 715.4470 & $1 \mathrm{ppm}$ \\
\hline $\mathrm{y} 7$ & 772.4677 & 772.4686 & $1 \mathrm{ppm}$ \\
\hline $\mathrm{y} 8$ & 903.5081 & 903.5049 & $3 \mathrm{ppm}$ \\
\hline $\mathrm{y} 9$ & 1016.592 & 1016.5834 & $8 \mathrm{ppm}$ \\
\hline Ppant ejection ion & 261.1267 & 261.1269 & $1 \mathrm{ppm}$ \\
\hline
\end{tabular}


Detail MS/MS data for Figure S5A

\begin{tabular}{|c|c|c|c|}
\hline & Calcd $m / z$ & Obsd $m / z$ & Error \\
\hline $\mathrm{b} 2$ & 201.1239 & 201.1233 & $3 \mathrm{ppm}$ \\
\hline $\mathrm{y} 2$ & 262.1514 & 262.1507 & $3 \mathrm{ppm}$ \\
\hline $\mathrm{y} 4$ & 474.3038 & 474.3027 & $2 \mathrm{ppm}$ \\
\hline $\mathrm{y} 5$ & 602.3623 & 602.3621 & $3 \mathrm{ppm}$ \\
\hline $\mathrm{y} 6$ & 715.4463 & 715.4460 & $4 \mathrm{ppm}$ \\
\hline $\mathrm{y} 7$ & 772.4677 & 772.4669 & $1 \mathrm{ppm}$ \\
\hline $\mathrm{y} 8$ & 903.5081 & 903.5062 & $2 \mathrm{ppm}$ \\
\hline $\mathrm{y} 9$ & 1016.592 & 1016.5920 & $0 \mathrm{ppm}$ \\
\hline Ppant ejection ion & 364.1359 & 364.1356 & $1 \mathrm{ppm}$ \\
\hline
\end{tabular}

Detail MS/MS data for Figure S5B

\begin{tabular}{|c|c|c|c|}
\hline & Calcd $m / z$ & Obsd $m / z$ & Error \\
\hline b2 & 201.1239 & 201.1225 & $7 \mathrm{ppm}$ \\
\hline $\mathrm{b} 4$ & 430.1936 & 430.1879 & $13 \mathrm{ppm}$ \\
\hline $\mathrm{y} 3$ & 361.2198 & 361.2177 & $6 \mathrm{ppm}$ \\
\hline $\mathrm{y} 4$ & 474.3038 & 474.3012 & $5 \mathrm{ppm}$ \\
\hline $\mathrm{y} 5$ & 602.3623 & 602.3595 & $5 \mathrm{ppm}$ \\
\hline $\mathrm{y} 6$ & 715.4463 & 715.4433 & $4 \mathrm{ppm}$ \\
\hline $\mathrm{y} 7$ & 772.4677 & 772.4642 & $4 \mathrm{ppm}$ \\
\hline $\mathrm{y} 8$ & 903.5081 & 903.5040 & $4 \mathrm{ppm}$ \\
\hline $\mathrm{y} 9$ & 1016.592 & 1016.5881 & $4 \mathrm{ppm}$ \\
\hline Ppant ejection ion & 421.1574 & 421.1555 & $4 \mathrm{ppm}$ \\
\hline
\end{tabular}


Detail MS/MS data for Figure S6A, i

\begin{tabular}{|c|c|c|c|}
\hline & Calcd $m / z$ & Obsd $m / z$ & Error \\
\hline $\mathrm{y} 3$ & 361.2198 & 361.2189 & $2 \mathrm{ppm}$ \\
\hline $\mathrm{y} 4$ & 474.3038 & 474.3031 & $1 \mathrm{ppm}$ \\
\hline $\mathrm{y} 5$ & 602.3623 & 602.3622 & $0.2 \mathrm{ppm}$ \\
\hline $\mathrm{y} 6$ & 715.4463 & 715.4452 & $1 \mathrm{ppm}$ \\
\hline $\mathrm{y} 7$ & 772.4677 & 772.4666 & $3 \mathrm{ppm}$ \\
\hline $\mathrm{y} 8$ & 903.5081 & 903.5055 & $1 \mathrm{ppm}$ \\
\hline y9 & 1016.592 & 1016.5910 & $1 \mathrm{ppm}$ \\
\hline Ppant ejection ion & 421.1574 & 421.1570 & \\
\hline
\end{tabular}

Detail MS/MS data for Figure S6A, ii

\begin{tabular}{|c|c|c|c|}
\hline & Calcd $m / z$ & Obsd $m / z$ & Error \\
\hline $\mathrm{b} 2$ & 201.1239 & 201.1225 & $7 \mathrm{ppm}$ \\
\hline $\mathrm{b3}$ & 258.1453 & 258.1435 & $7 \mathrm{ppm}$ \\
\hline $\mathrm{y} 3$ & 361.2198 & 361.2168 & $8 \mathrm{ppm}$ \\
\hline $\mathrm{y} 4$ & 474.3038 & 474.3021 & $3 \mathrm{ppm}$ \\
\hline $\mathrm{y} 5$ & 602.3623 & 602.3593 & $5 \mathrm{ppm}$ \\
\hline $\mathrm{y} 6$ & 715.4463 & 715.4423 & $5 \mathrm{ppm}$ \\
\hline $\mathrm{y} 7$ & 772.4677 & 772.4636 & $5 \mathrm{ppm}$ \\
\hline $\mathrm{y} 8$ & 903.5081 & 903.5048 & $4 \mathrm{ppm}$ \\
\hline $\mathrm{y} 9$ & 1016.592 & 1016.6003 & $8 \mathrm{ppm}$ \\
\hline Ppant ejection ion & 420.1257 & 420.1234 & $5 \mathrm{ppm}$ \\
\hline
\end{tabular}


Detail MS/MS data for Figure S6B, i

\begin{tabular}{|c|c|c|c|}
\hline & Calcd $m / z$ & Obsd $m / z$ & Error \\
\hline $\mathrm{b} 2$ & 201.1239 & 201.1227 & $6 \mathrm{ppm}$ \\
\hline $\mathrm{b} 3$ & 315.1667 & 315.1646 & $5 \mathrm{ppm}$ \\
\hline $\mathrm{y} 3$ & 361.2198 & 361.2180 & $4 \mathrm{ppm}$ \\
\hline $\mathrm{y} 4$ & 474.3038 & 474.3016 & $4 \mathrm{ppm}$ \\
\hline $\mathrm{y} 5$ & 602.3623 & 602.3599 & $4 \mathrm{ppm}$ \\
\hline $\mathrm{y} 6$ & 715.4463 & 715.4435 & $4 \mathrm{ppm}$ \\
\hline $\mathrm{y} 7$ & 772.4677 & 772.4647 & $4 \mathrm{ppm}$ \\
\hline $\mathrm{y} 8$ & 903.5081 & 903.5048 & $4 \mathrm{ppm}$ \\
\hline $\mathrm{y} 9$ & 1016.592 & 1016.5881 & $4 \mathrm{ppm}$ \\
\hline Ppant ejection ion & 425.1646 & 425.1629 & \\
\hline
\end{tabular}

Detail MS/MS data for Figure S6B, ii

\begin{tabular}{|c|c|c|c|}
\hline & Calcd $m / z$ & Obsd $m / z$ & Error \\
\hline b2 & 201.1239 & 201.1226 & $6 \mathrm{ppm}$ \\
\hline $\mathrm{b3}$ & 315.1667 & 315.1642 & $8 \mathrm{ppm}$ \\
\hline $\mathrm{y} 3$ & 361.2198 & 361.2181 & $5 \mathrm{ppm}$ \\
\hline $\mathrm{y} 4$ & 474.3038 & 474.3016 & $5 \mathrm{ppm}$ \\
\hline $\mathrm{y} 5$ & 602.3623 & 602.3597 & $4 \mathrm{ppm}$ \\
\hline $\mathrm{y} 6$ & 715.4463 & 715.4440 & $3 \mathrm{ppm}$ \\
\hline $\mathrm{y} 7$ & 772.4677 & 772.4651 & $3 \mathrm{ppm}$ \\
\hline $\mathrm{y} 8$ & 903.5081 & 903.5044 & $4 \mathrm{ppm}$ \\
\hline y9 & 1016.592 & 1016.5879 & $4 \mathrm{ppm}$ \\
\hline Ppant ejection ion & 423.1360 & 423.1341 & $4 \mathrm{ppm}$ \\
\hline
\end{tabular}


Detail MS/MS data for Figure S6C, $\mathbf{i}$

\begin{tabular}{|c|c|c|c|}
\hline & Calcd $m / z$ & Obsd $m / z$ & Error \\
\hline $\mathrm{b} 2$ & 201.1239 & 201.1227 & $6 \mathrm{ppm}$ \\
\hline $\mathrm{y} 3$ & 361.2198 & 361.2181 & $5 \mathrm{ppm}$ \\
\hline $\mathrm{y} 4$ & 474.3038 & 474.3016 & $5 \mathrm{ppm}$ \\
\hline $\mathrm{y} 5$ & 602.3623 & 602.3602 & $3 \mathrm{ppm}$ \\
\hline $\mathrm{y} 6$ & 715.4463 & 715.4440 & $3 \mathrm{ppm}$ \\
\hline $\mathrm{y} 7$ & 772.4677 & 772.4651 & $3 \mathrm{ppm}$ \\
\hline $\mathrm{y} 8$ & 903.5081 & 903.5044 & $4 \mathrm{ppm}$ \\
\hline $\mathrm{y} 9$ & 1016.592 & 1016.5883 & $3 \mathrm{ppm}$ \\
\hline Ppant ejection ion & 424.1762 & 424.1747 & $3 \mathrm{ppm}$ \\
\hline
\end{tabular}

Detail MS/MS data for Figure S6C, ii

\begin{tabular}{|c|c|c|c|}
\hline & Calcd $m / z$ & Obsd $m / z$ & Error \\
\hline b2 & 201.1239 & 201.1227 & $6 \mathrm{ppm}$ \\
\hline b5 & 430.1936 & 430.2017 & $18 \mathrm{ppm}$ \\
\hline $\mathrm{y} 3$ & 361.2198 & 361.2181 & $5 \mathrm{ppm}$ \\
\hline y4 & 474.3038 & 474.3021 & $3 \mathrm{ppm}$ \\
\hline $\mathrm{y} 5$ & 602.3623 & 602.3597 & $4 \mathrm{ppm}$ \\
\hline $\mathrm{y} 6$ & 715.4463 & 715.4438 & $3 \mathrm{ppm}$ \\
\hline y7 & 772.4677 & 772.4651 & $3 \mathrm{ppm}$ \\
\hline y8 & 903.5081 & 903.5052 & $3 \mathrm{ppm}$ \\
\hline y9 & 1016.592 & 1016.5890 & $3 \mathrm{ppm}$ \\
\hline Ppant ejection ion & 420.1257 & 420.1244 & $3 \mathrm{ppm}$ \\
\hline
\end{tabular}


Detail MS/MS data for Figure S8A

\begin{tabular}{|c|c|c|c|}
\hline & Calcd $m / z$ & Obsd $m / z$ & Error \\
\hline b2 & 201.1239 & 201.1230 & $5 \mathrm{ppm}$ \\
\hline $\mathrm{b} 5$ & 430.1936 & 430.2122 & $40 \mathrm{ppm}$ \\
\hline $\mathrm{y} 3$ & 361.2198 & 361.2185 & $4 \mathrm{ppm}$ \\
\hline $\mathrm{y} 4$ & 474.3038 & 474.3023 & $3 \mathrm{ppm}$ \\
\hline $\mathrm{y} 5$ & 602.3623 & 602.3608 & $2 \mathrm{ppm}$ \\
\hline $\mathrm{y} 6$ & 715.4463 & 715.4449 & $2 \mathrm{ppm}$ \\
\hline $\mathrm{y} 7$ & 772.4677 & 772.5064 & $50 \mathrm{ppm}$ \\
\hline $\mathrm{y} 8$ & 903.5081 & 903.5052 & $3 \mathrm{ppm}$ \\
\hline $\mathrm{y} 9$ & 1016.592 & 1016.5903 & $2 \mathrm{ppm}$ \\
\hline Ppant ejection ion & 421.1574 & 421.1565 & $2 \mathrm{ppm}$ \\
\hline
\end{tabular}

Detail MS/MS data for Figure S8B

\begin{tabular}{|c|c|c|c|}
\hline & Calcd $m / z$ & Obsd $m / z$ & Error \\
\hline $\mathrm{b} 2$ & 201.1239 & 201.1231 & $4 \mathrm{ppm}$ \\
\hline $\mathrm{y} 1$ & 175.1194 & 175.1187 & $4 \mathrm{ppm}$ \\
\hline $\mathrm{y} 3$ & 361.2198 & 361.2185 & $3 \mathrm{ppm}$ \\
\hline $\mathrm{y} 4$ & 474.3038 & 474.3025 & $2 \mathrm{ppm}$ \\
\hline $\mathrm{y} 5$ & 602.3623 & 602.3610 & $2 \mathrm{ppm}$ \\
\hline $\mathrm{y} 6$ & 715.4463 & 715.4457 & $1 \mathrm{ppm}$ \\
\hline $\mathrm{y} 7$ & 772.4677 & 772.4665 & $1 \mathrm{ppm}$ \\
\hline $\mathrm{y} 8$ & 903.5081 & 903.5069 & $1 \mathrm{ppm}$ \\
\hline $\mathrm{y} 9$ & 1016.592 & 1016.5907 & $1 \mathrm{ppm}$ \\
\hline Ppant ejection ion & 421.1574 & 421.1566 & $2 \mathrm{ppm}$ \\
\hline
\end{tabular}


Detail MS/MS data for Figure S9A

\begin{tabular}{|c|c|c|c|}
\hline & Calcd $m / z$ & Obsd $m / z$ & Error \\
\hline b2 & 201.1239 & 201.1226 & $6 \mathrm{ppm}$ \\
\hline $\mathrm{b} 4$ & 315.1667 & 315.1654 & $4 \mathrm{ppm}$ \\
\hline $\mathrm{y} 3$ & 361.2198 & 361.2197 & $0.5 \mathrm{ppm}$ \\
\hline $\mathrm{y} 4$ & 474.3038 & 474.3040 & $0.5 \mathrm{ppm}$ \\
\hline $\mathrm{y} 5$ & 602.3623 & 602.3593 & $5 \mathrm{ppm}$ \\
\hline $\mathrm{y} 6$ & 715.4463 & 715.4444 & $3 \mathrm{ppm}$ \\
\hline $\mathrm{y} 7$ & 772.4677 & 772.4638 & $5 \mathrm{ppm}$ \\
\hline $\mathrm{y} 8$ & 903.5081 & 903.5040 & $5 \mathrm{ppm}$ \\
\hline $\mathrm{y} 9$ & 1016.592 & 1016.5868 & $5 \mathrm{ppm}$ \\
\hline Ppant ejection ion & 459.1697 & 459.1675 & $5 \mathrm{ppm}$ \\
\hline
\end{tabular}

Detail MS/MS data for Figure S9B

\begin{tabular}{|c|c|c|c|}
\hline & Calcd $m / z$ & Obsd $m / z$ & Error \\
\hline $\mathrm{b} 2$ & 201.1239 & 201.1233 & $3 \mathrm{ppm}$ \\
\hline $\mathrm{Y} 2$ & 262.1514 & 262.1513 & $0.5 \mathrm{ppm}$ \\
\hline $\mathrm{y} 3$ & 361.2198 & 361.2206 & $2 \mathrm{ppm}$ \\
\hline $\mathrm{y} 4$ & 474.3038 & 474.3079 & $9 \mathrm{ppm}$ \\
\hline $\mathrm{y} 5$ & 602.3623 & 602.3608 & $1 \mathrm{ppm}$ \\
\hline $\mathrm{y} 6$ & 715.4463 & 715.4468 & $0.5 \mathrm{ppm}$ \\
\hline $\mathrm{y} 7$ & 772.4677 & 772.4681 & $3 \mathrm{ppm}$ \\
\hline $\mathrm{y} 8$ & 903.5081 & 903.5107 & $3 \mathrm{ppm}$ \\
\hline y9 & 1016.592 & 1016.5889 & $2 \mathrm{ppm}$ \\
\hline Ppant ejection ion & 459.1697 & 459.1704 & \\
\hline
\end{tabular}




\section{SUPPLEMENTARY REFERENCES}

Green, M. R. \& Sambrook, J. Molecular Cloning: A Laboratory Manual (Cold Spring Harbor Laboratory Press, 2012).

Kieser, T., Bibb, M. J., Buttner, M. J., Chater, K. F. \& Hopwood, D. A. Practical Streptomyces Genetics. (John Innes Foundation, 2000).

Li, D. et al. pFind: a novel database-searching software system for automated peptide and protein identification via tandem mass spectrometry. Bioinformatics 21, 3049-3050 (2005).

Chen, M., Pang, B., Du, Y. N., Zhang, Y. P. \& Liu, W. Characterization of the metallo-dependent amidohydrolases responsible for "auxiliary" leucinyl removal in the biosynthesis of 2,2'bipyridine antibiotics. Synth Syst Biotechnol 2, 137-146 (2017).

Wei, C. et al. Dual-Reactable Fluorescent Probes for Highly Selective and Sensitive Detection of Biological $\mathrm{H}_{2}$ S. Chem. Asian J. 11, 1376-1381 (2016).

Bumpus, S. B. \& Kelleher, N. L. Accessing natural product biosynthetic processes by mass spectrometry. Curr. Opin. Chem. Biol. 12, 475-482 (2008).

$\mathrm{Qu}, \mathrm{X}$. et al. Caerulomycins and collismycins share a common paradigm for 2,2'-bipyridine biosynthesis via an unusual hybrid polyketide-peptide assembly Logic. J. Am. Chem. Soc. 134, 9038-9041 (2012).

Watanabe, K., Khosla, C., Stroud, R. M. \& Tsai, S.-C. Crystal Structure of an Acyl-ACP Dehydrogenase from the FK520 Polyketide Biosynthetic Pathway: Insights into Extender Unit Biosynthesis. J. Mol. Biol. 334, 435-444 (2003).

Toogood, H. S. et al. Extensive domain motion and electron transfer in the human electron transferring flavoprotein.medium chain Acyl-CoA dehydrogenase complex. J. Biol. Chem. 279, 32904-32912 (2004).

Battaile, K. P. et al. Crystal structure of rat short chain acyl-CoA dehydrogenase complexed with acetoacetyl-CoA: comparison with other acyl-CoA dehydrogenases. J. Biol. Chem. 277, 12200-12207 (2002).

1 Kung, J. W., Seifert, J., von Bergen, M. \& Boll, M. Cyclohexanecarboxyl-coenzyme A (CoA) and cyclohex-1-ene-1-carboxyl-CoA dehydrogenases, two enzymes involved in the fermentation of benzoate and crotonate in Syntrophus aciditrophicus. J. Bacteriol. 195, 3193 3200 (2013).

Mootz, H. D. \& Marahiel, M. A. The tyrocidine biosynthesis operon of Bacillus brevis: complete nucleotide sequence and biochemical characterization of functional internal adenylation domains. J. Bacteriol. 179, 6843-6850 (1997).

Konz, D., Klens, A., Schörgendorfer, K. \& Marahiel, M. A. The bacitracin biosynthesis operon of Bacillus licheniformis ATCC 10716: molecular characterization of three multi-modular peptide synthetases. Chem. Biol. 4, 927-937 (1997).

Duitman, E. H. et al. The mycosubtilin synthetase of Bacillus subtilis ATCC6633: a multifunctional hybrid between a peptide synthetase, an amino transferase, and a fatty acid synthase. Proc. Natl. Acad. Sci. U. S. A. 96, 13294-13299 (1999).

Molnár, I. et al. The biosynthetic gene cluster for the microtubule-stabilizing agents epothilones A and B from Sorangium cellulosum So ce90. Chem. Biol. 7, 97-109 (2000).

Gaudelli, N. M., Long, D. H. \& Townsend, C. A. beta-Lactam formation by a non-ribosomal 
peptide synthetase during antibiotic biosynthesis. Nature 520, 383-387 (2015).

Hojati, Z. et al. Structure, Biosynthetic Origin, and Engineered Biosynthesis of CalciumDependent Antibiotics from Streptomyces coelicolor. Chem. Biol. 9, 1175-1187 (2002).

Chiu, H. T. et al. Molecular cloning and sequence analysis of the complestatin biosynthetic gene cluster. Proc. Natl. Acad. Sci. U. S. A. 98, 8548-8553 (2001).

19 Steller, S. et al. Structural and functional organization of the fengycin synthetase multienzyme system from Bacillus subtilis b213 and A1/3. Chem. Biol. 6, 31-41 (1999).

20 Turgay, K., Krause, M. \& Marahiel, M. A. Four homologous domains in the primary structure of GrsB are related to domains in a superfamily of adenylate-forming enzymes. Mol. Microbiol. 6, 529-546 (1992).

21 Tsuge, K., Akiyama, T. \& Shoda, M. Cloning, sequencing, and characterization of the iturin A operon. J. Bacteriol. 183, 6265-6273 (2001).

22 Konz, D., Doekel, S. \& Marahiel, M. A. Molecular and biochemical characterization of the protein template controlling biosynthesis of the lipopeptide lichenysin. J. Bacteriol. 181, 133140 (1999).

23 Bamas-Jacques, N., Lorenzon, S., Lacroix, P., de Swetschin, C. \& Crouzet, J. Cluster organization of the genes of Streptomyces pristinaespiralis involved in pristinamycin biosynthesis and resistance elucidated by pulsed-field gel electrophoresis. J. Appl. Microbiol. 87, 939-948 (1999).

24 Mast, Y. et al. Characterization of the 'pristinamycin supercluster' of Streptomyces pristinaespiralis. Microb Biotechnol 4, 192-206 (2011).

25 Ziemert, N. et al. The natural product domain seeker NaPDoS: a phylogeny based bioinformatic tool to classify secondary metabolite gene diversity. PLoS One 7, e34064 (2012).

26 Rausch, C., Hoof, I., Weber, T., Wohlleben, W. \& Huson, D. H. Phylogenetic analysis of condensation domains in NRPS sheds light on their functional evolution. BMC Evol. Biol. 7, 78-92 (2007).

27 Schauwecker, F., Pfennig, F., Schroder, W. \& Keller, U. Molecular cloning of the actinomycin synthetase gene cluster from Streptomyces chrysomallus and functional heterologous expression of the gene encoding actinomycin synthetase II. J. Bacteriol. 180, 2468-2474 (1998).

28 Roongsawang, N. et al. Cloning and characterization of the gene cluster encoding arthrofactin synthetase from Pseudomonas sp MIS38. Chem. Biol. 10, 869-880 (2003).

29 Pogorevc, D. et al. Biosynthesis and Heterologous Production of Argyrins. Acs Synth Biol 8, 1121-1133 (2019).

30 Yuwen, L. et al. The role of aromatic L-amino acid decarboxylase in bacillamide C biosynthesis by Bacillus atrophaeus C89. Sci. Rep. 3, 1753-1762 (2013).

31 Du, L., Sánchez, C., Chen, M., Edwards, D. J. \& Shen, B. The biosynthetic gene cluster for the antitumor drug bleomycin from Streptomyces verticillus ATCC15003 supporting functional interactions between nonribosomal peptide synthetases and a polyketide synthase. Chem. Biol. 7, 623-642 (2000).

32 Homburg, S., Oswald, E., Hacker, J. \& Dobrindt, U. Expression analysis of the colibactin gene cluster coding for a novel polyketide in Escherichia coli. FEMS Microbiol. Lett. 275, 255-262 (2007).

33 Gehring, A. M., Mori, I. \& Walsh, C. T. Reconstitution and characterization of the Escherichia coli enterobactin synthetase from EntB, EntE, and EntF. Biochemistry 37, 2648-2659 (1998). 
34 Gehring, A. M. et al. Iron acquisition in plague: modular logic in enzymatic biogenesis of yersiniabactin by Yersinia pestis. Chem. Biol. 5, 573-586 (1998).

35 Nishizawa, T. et al. Polyketide synthase gene coupled to the peptide synthetase module involved in the biosynthesis of the cyclic heptapeptide microcystin. J. Biochem. 127, 779-789 (2000).

36 Nowak-Thompson, B., Chaney, N., Wing, J. S., Gould, S. J. \& Loper, J. E. Characterization of the pyoluteorin biosynthetic gene cluster of Pseudomonas fluorescens Pf-5. J. Bacteriol. 181, 2166-2174 (1999).

37 Chen, J.-S. et al. Functional identification of the gene encoding the enzyme involved in mannosylation in ramoplanin biosynthesis in Actinoplanes sp. Biotechnol. Lett 35, 1501-1508 (2013).

38 Bruner, S. D. et al. Structural basis for the cyclization of the lipopeptide antibiotic surfactin by the thioesterase domain SrfTE. Structure 10, 301-310 (2002).

39 Scholz-Schroeder, B. K., Soule, J. D. \& Gross, D. C. The sypA, sypB and sypC synthetase genes encode twenty-two modules involved in the nonribosomal peptide synthesis of syringopeptin by Pseudomonas syringae pv. syringae B301D. Mol. Plant. Microbe Interact. 16, 271-280 (2003).

40 Pootoolal, J. et al. Assembling the glycopeptide antibiotic scaffold: The biosynthesis of A47934 from Streptomyces toyocaensis NRRL 15009. Proc. Natl. Acad. Sci. U. S. A. 99, 8962-8967 (2002).

41 Guenzi, E., Galli, G., Grgurina, I., Gross, D. C. \& Grandi, G. Characterization of the syringomycin synthetase gene cluster - A link between prokaryotic and eukaryotic peptide synthetases. J. Biol. Chem. 273, 32857-32863 (1998).

42 Lombo, F. et al. Deciphering the biosynthesis pathway of the antitumor thiocoraline from a marine actinomycete and its expression in two Streptomyces species. Chembiochem 7, 366376 (2006).

43 Wyckoff, E. E., Stoebner, J. A., Reed, K. E. \& Payne, S. M. Cloning of a Vibrio cholerae vibriobactin gene cluster: Identification of genes required for early steps in siderophore biosynthesis. J. Bacteriol. 179, 7055-7062 (1997).

44 Pfeifer, B. A., Admiraal, S. J., Gramajo, H., Cane, D. E. \& Khosla, C. Biosynthesis of complex polyketides in a metabolically engineered strain of E. coli. Science 291, 1790-1792 (2001).

45 Fang, J. et al. Cloning and characterization of the tetrocarcin A gene cluster from Micromonospora chalcea NRRL 11289 reveals a highly conserved strategy for tetronate biosynthesis in spirotetronate antibiotics. J. Bacteriol. 190, 6014-6025 (2008). 